\title{
CHARACTERISTIC COHOMOLOGY OF DIFFERENTIAL SYSTEMS (I): GENERAL THEORY
}

\author{
ROBERT L. BRYANT AND PHILLIP A. GRIFFITHS
}

\section{CONTENTS}

Introduction

1. Basic considerations and first examples

1.1. Definition of characteristic cohomology

1.2. The exterior differential system associated to a partial differential equation; symmetries

1.3. The prolongation tower

1.4. Variation of characteristic cohomology

2. Frobenius extensions

2.1. Structure equations of the prolongation tower

2.2. The symbol relations

2.3. Frobenius extensions

2.4. The weight filtration

2.5. A variant of Spencer cohomology

3. $\Delta$-multilinear and commutative algebra

3.1. Poincaré polynomials and Spencer cohomology

3.2. $\Delta$-tensor and exterior products

4. Vanishing of the characteristic cohomology

4.1. Characteristic cohomology in the unconstrained case

4.2. Involutivity and $\Delta$-exterior powers

4.3. The tableau and its normal form

4.4. The determined case

4.5. The unmixed, overdetermined case

4.6. The mixed case

5. Structure of the characteristic cohomology

5.1. A variant of the Spencer complex

5.2. Quadratic conservation laws for constant coefficient linear equations

Received by the editors July 21, 1993.

1991 Mathematics Subject Classification. Primary 58A15, 35A30; Secondary 35L65, 58H 10. 
6. Global results

6.1. A relation between ordinary and characteristic cohomology

6.2. $\mathscr{I}$-homology

6.3. Moment conditions

\section{References}

\section{INTRODUCTION}

In this first of a series of papers, we shall introduce and begin to study and apply the notion of the characteristic cohomology of an exterior differential system. Recall that an exterior differential system is a graded, differentially closed ideal $\mathscr{I}_{0} \subset \Omega^{*}\left(X_{0}\right)$ on a manifold $X_{0}$. It is natural to consider the cohomology of the quotient complex $\bar{\Omega}_{0}^{*}=\Omega^{*}\left(X_{0}\right) / \mathscr{I}_{0}$. However, this cohomology turns out not to have good functorial properties. For this and other reasons to be explained below, we shall usually pass to the infinite prolongation $(X, \mathscr{I})$ of $\left(X_{0}, \mathscr{I}_{0}\right)$, and define the characteristic cohomology $\bar{H}^{*}$ of $\left(X_{0}, \mathscr{J}_{0}\right)$ to be the cohomology of the quotient complex $\bar{\Omega}^{*}=\Omega^{*}(X) / \mathscr{I}$. Our first general result is that, in the local involutive case,

$$
\bar{H}^{q}=0 \text { for } 0<q<n-\ell,
$$

where $n$ is the dimension of the maximal integral manifolds (solutions) of the exterior differential system and $\ell$ is an easily computed integer which measures the "degree of overdeterminedness" of $\mathscr{I}_{0}$. (When $\mathscr{J}_{0}$ is "unmixed"-i.e., roughly speaking, it is not composed of several exterior differential systems of different degress of complexity- $\ell$ is the codimension of the characteristic variety.) The first non-zero group $\bar{H}^{n-\ell}$ turns out to have a structure not immediately apparent from its definition. For reasons to be discussed below, we define the space $\mathscr{C}$ of conservation laws of the exterior differential system to be $\bar{H}^{n-\ell}$. As we shall see, in all of the cases of exterior differential systems which model classical partial differential equations, this space turns out to be isomorphic to the space of conservation laws as they are generally understood.

For exterior differential systems arising from a system of partial differential equations, this result builds on and generalizes extensive previous work (see the references below). However, a major difference in our viewpoint is that, throughout, we insist on full contact invariance of the constructions. Thus, PDEs which appear to be quite different (such as $u_{x x} u_{y y}-u_{x y}^{2}=1$ and $\left.u_{x x}-u_{y y}=0\right)$ may still be contact equivalent and therefore have the same characteristic cohomology. Since we are not carrying along unnecessary additional structure, such as that needed to keep track of point invariance, the theory perhaps has greater conceptual simplicity.

We are especially interested in developing methods for effectively computing $\mathscr{C}$ in examples, and in Part II we begin this task. We are especially interested in exterior differential systems that arise from questions in geometry; for example, the study of gradient flows of functionals defined on classes of immersions which themselves are subject to differential constraints. Now, such exterior differential systems may always be written in local coordinates as PDE systems, but there is generally no preferred coordinate system and the introduction of 
arbitrarily chosen coordinates frequently obscures the geometry. Even when there are more-or-less natural coordinates, the computation of the conservation laws of a given system has generally relied either on symmetry considerations or inspired guesswork. One of our goals is to provide a complementary, in some cases more systematic, method.

Our proof of the general result (1) has essentially two steps. The first is to consider the spectral sequence of the filtered complex $F^{p} \Omega^{*}$, where

$$
F^{p} \Omega^{*}=\text { image }\{\underbrace{\mathscr{I} \wedge \cdots \wedge \mathscr{I}}_{p \text { times }} \wedge \Omega^{*}(X) \rightarrow \Omega^{*}(X)\} .
$$

This spectral sequence abuts to $H^{*}(X)$ and the characteristic cohomology is the term $E_{1}^{0, *}$. As will now be explained, invariants of the exterior differential system, such as its symbol, appear naturally in calculation of the terms $E_{1}^{p \text {,* }}$ for $p>0$. Using information about these higher degree groups and standard spectral sequence machinery will then allow us to "solve" for the characteristic cohomology in this spectral sequence.

The study of the terms $E_{1}^{p, *}$ for fixed $p>0$ involves a construction from exterior differential algebra that we shall call Frobenius extension. One thinks of a completely integrable Pfaffian system as being the simplest type of exterior differential system, and Frobenius extensions can be thought of as an attempt to close up a given Pfaffian system relative to the Frobenius integrability condition by adjoining new 1 -forms. The actual definition of Frobenius extension is given in Section 2.3, as well as that of a Frobenius tower, which is an iteration of Frobenius extensions. The importance of this concept is that the prolongation tower of an involutive exterior differential system is captured algebraically by the notion of a Frobenius tower. For $p>0$ the "position" of a form in the Frobenius tower allows us to define a weight filtration $W_{k} \bar{\Omega}^{p, *}$ on the complexes used to compute $E_{1}^{p, *}$, and then the main observation is that on the associated graded complex

$$
\bar{\Omega}_{k}^{p, *}=W_{k} \bar{\Omega}^{p, *} / W_{k-1} \bar{\Omega}^{p, *}
$$

the induced differential $\delta$ is linear over the functions. The cohomology

$$
H\left(\bar{\Omega}_{k}^{p, *}, \delta\right), \quad p>0,
$$

is therefore algebraic and it is at this point that the symbol of the exterior differential system makes its appearance. For $p=1$ the cohomology (2) turns out to be a variant of classical Spencer cohomology, but for $p \geqq 2$ a new algebraic object $\Lambda_{\Delta}^{p} M$ appears.

The second step then consists of the study of the $\Lambda_{\Delta}^{p} M$. Over a polynomial ring $S=\mathbb{F}\left[x^{1}, \ldots, x^{n}\right]$ where $\mathbb{F}$ is any field of characteristic zero, we consider $S$-modules $M=\bigoplus_{k \geqq k_{0}} M_{k}$ which are quasi-finitely generated in the sense that $\operatorname{dim}_{\mathbb{F}} M_{k}<\infty$ for all $k$. The $\Delta$-tensor product $M \otimes_{\Delta} N$ of two such modules is defined as a graded $\mathbb{F}$-vector space by $M \otimes_{\Delta} N=M \otimes_{\mathbb{F}} N$ and the $S$-module structure is defined by the rule

$$
x^{i}\left(m \otimes_{\Delta} n\right)=x^{i} m \otimes_{\Delta} n+m \otimes_{\Delta} x^{i} n .
$$


From the $\Delta$-tensor powers $\otimes_{\Delta}^{p} M$ we may define the $\Delta$-exterior powers $\Lambda_{\Delta}^{p} M$ and our main algebraic result is: If $M$ is an involutive $S$-module with resolution length $\ell(M)$, then $\Lambda_{\Delta}^{p} M$ is involutive with resolution length $\ell\left(\Lambda_{\Delta}^{p} M\right) \leqq \ell(M)$. In spite of its "functorial" appearance, this result does not seem to admit of a simple proof, but involves a careful study of the defining relations of the modules in question. That this result is not entirely trivial is suggested by the observation that if the symbol is "split" then the minimal resolution of $M$ essentially lifts to one of the $\Lambda_{\Delta}^{p} M$, whereas if the symbol is generic then $\Lambda_{\Delta}^{p} M$ is free in the lower degrees and in general is "more free" than $M$.

The integer $\ell$ in (1) turns out to be the resolution length of the symbol module $M$ associated to the exterior differential system, and the above vanishing result ultimately follows from the commutative algebra vanishing result that

$$
H_{\mathrm{Kos}}^{q}\left(\Lambda_{\Delta}^{p} M\right)=0, \quad p>0 \text { and } 0 \leqq q<n-\ell,
$$

where $H_{\mathrm{Kos}}^{q}$ is the Poincare dual of ordinary Koszul homology.

The quotient group $\bar{H}^{n-\ell}$ turns out to have a more concrete realization than just as a cohomology group. This comes from the fact that there are canonically defined vector bundles $E_{0}$ and $E_{1}$ (whose fibres are Spencer-type cohomology groups) and a canonical linear differential operator

$$
\nabla: E_{0} \rightarrow E_{1}
$$

such that

$$
\bar{H}^{n-\ell} \cong \operatorname{ker} \nabla
$$

Thus this cohomology, which is defined as a quotient space, is alternatively given as the kernel of an intrinsic differential operator. In the classical or unconstrained case $\ell=0$, the group $\bar{H}^{n}$ is the space of equivalence classes of functionals on integral manifolds and (3) represents a functional by its EulerLagrange equation. The general case is an extension of this concept.

This explicit realization of the space of conservation laws as the kernel of a (generally overdetermined) differential operator will be seen to have important consequences. In particular, one application of (3) is to show that the space of translation-invariant quadratic conservation laws for a determined, linear constant-coefficient PDE system is given by $\operatorname{Sym}_{\Delta}^{2}(M)$, where $M$ is the module associated to the symbol of the equation.

Finally, in Section 6 we will discuss some topological issues related to (1). The first is a straightforward globalization of (1), expressed by the statements

$$
\begin{aligned}
& H^{q}(X, \mathbb{R}) \cong \bar{H}^{q}(X), \quad 0 \leqq q<n-\ell \\
& 0 \rightarrow H^{n-\ell}(X, \mathbb{R}) \rightarrow \bar{H}^{n-\ell}(X) \rightarrow H^{0}\left(\overline{\mathscr{H}}^{n-\ell}\right)
\end{aligned}
$$

where $\overline{\mathscr{H}}^{n-\ell}$ is the sheaf of conservation laws. This result suggests certain purely geometric considerations. If we define $H_{*, \mathscr{F}}(X)$ to be the homology computed from the complex of piecewise- $C^{1}$ chains whose individual simplices 
are integral manifolds of $\mathscr{I}$, then (i) and (ii) suggest the statements

$$
\begin{aligned}
& H_{q, \mathscr{I}}(X) \cong H_{q}(X), \quad 0 \leqq q<n-\ell, \\
& H_{n-\ell, \mathscr{I}}(X) \rightarrow H_{n-\ell}(X) \rightarrow 0 .
\end{aligned}
$$

Both of these would follow from the local result

$$
H_{q, \mathscr{I}}(X)=0, \quad 0 \leqq q<n-\ell,
$$

where $X$ is a sufficiently small neighborhood of an ordinary integral element. Now (v) is in turn closely related to a result of Thom [10], and in Section 6 we present a sketch of how (v) might follow from Thom's arguments in case $\mathscr{I}$ has no real Cartan characteristics in the range $0 \leqq q<n-\ell$. In general however, the real Cartan characteristics will contribute singularity considerations which Thom's arguments do not seem to be able to address, thus we only pose (v) as a question.

Especially interesting is the local group $H_{n-\ell, \mathscr{I}}(X)$ and the natural mapping

$$
\mu: \mathscr{C} \rightarrow \operatorname{Hom}\left(H_{n-\ell, \mathscr{I}}(X), \mathbb{R}\right)
$$

under which conservation laws give what are called moment conditions. We conclude this section by analyzing $\mu$ in a number of examples. It is clear that the study of the postulated " $\mathscr{I}$-de-Rham theorem"-meaning the analysis of whether the natural pairing

$$
\bar{H}^{k}(X) \otimes H_{k, \mathscr{I}}(X) \rightarrow \mathbb{R}
$$

is non-degenerate (even locally)-is extremely interesting. The discussion in Section 6 raises more questions than it answers and, in our opinion, points out a very fruitful area for further work.

The principal applications of the general theory will be given in subsequent papers. In particular, in Part II we shall completely analyze the conservation laws for a class of exterior differential systems that we call parabolic systems. As we shall see there the general theory will serve to "guide" the application of E. Cartan's equivalence method. This method gives in principle an algorithm for determining the intrinsic invariants of an exterior differential system. However, as in classical invariant theory the calculations very quickly get out of hand unless one is studying a situation that is guided by a geometric problem. It is such a guide that is provided by the general theory.

This work has been principally influenced by the papers [13], [14] and [15] of Vinogradov. In seeking to understand the characteristic cohomology of an exterior differential system we were led to what is now called the "Vinogradov spectral sequence of the variational bicomplex" in the context of an exterior differential system. In this setting there is no longer a bicomplex, but rather there is a filtered complex. By insisting on full contact invariance of the theory one is led naturally to the Frobenius extension construction mentioned above and its cohomological implications.

The papers of Vinogradov built on earlier work of the Russian school of formal differential geometry initiated by Gelfand and his collaborators (see [5] and Manin [8]). There is by now a considerable literature on these subjects, 
and although our context is quite different we should like to mention Olver [9], Tsujishita [11], Anderson [1] and Dickey [4] as sources for further work and additional references to the literature. In particular, in the case when an exterior differential system arises from a determined PDE system in CauchyKowaleska form, our main result implies the so-called "two-line theorem" of Vinogradov. Extensions of this result, still in the context of the variational bicomplex, to some more general classes of PDE's have independently been given by Tsujishita [12] and Anderson (loc. cit.).

\section{BASIC CONSIDERATIONS AND FIRST EXAMPLES}

1.1. Definition of characteristic cohomology. Let $X_{0}$ be a smooth manifold and $\Omega^{*}\left(X_{0}\right)=\bigoplus_{p \geqq 0} \Omega^{p}\left(X_{0}\right)$ the differential graded algebra of smooth differential forms on $X_{0}$. We recall that an exterior differential system is given by a homogeneous differential ideal $\mathscr{I}_{0} \subset \Omega^{*}\left(X_{0}\right)$. These two conditions mean that $\mathscr{I}_{0}=\bigoplus_{q \geqq 0} \mathscr{J}_{0}^{q}$, where $\mathscr{J}_{0}^{q}=\mathscr{I}_{0} \cap \Omega^{q}\left(X_{0}\right)$ and $d \mathscr{I}_{0} \subseteq \mathscr{I}_{0}$. We shall follow the terminology, and, for the most part, the notations of [2]. We shall also be assuming familiarity with the background of results from [2].

We shall be interested in the integral manifolds of $\mathscr{J}_{0}$. By an integral manifold we shall mean a smooth immersion

$$
f: N \rightarrow X
$$

where $f^{*} \theta=0, \theta \in \mathscr{I}_{0}$. Intuitively, integral manifolds are solutions to the PDE system

$$
\theta=0 \text { where } \theta \in \mathscr{I}_{0},
$$

and where to "solve the equation $\theta=0$ " means to find a submanifold on which $\theta$ restricts to zero. We will in particular be interested in integral manifolds of dimension $n$, and for purposes of exposition shall make the assumptions

$$
\left\{\begin{array}{l}
\mathscr{J}_{0}^{0}=(0), \\
\mathscr{J}_{0}^{q}=\Omega^{q}\left(X_{0}\right) \text { for } q>n .
\end{array}\right.
$$

The first assumption means that $\mathscr{J}_{0}$ contains no functions-otherwise we may replace $X_{0}$ by the subset, assumed to be a submanifold, defined by setting equal to zero all of the functions in $\mathscr{J}_{0}$. Neither assumption changes the set of $n$-dimensional integral manifolds.

The very definition of an exterior differential system suggests that we consider the complex $\left\{\bar{\Omega}_{0}^{*}, \bar{d}\right\}$ where

$$
\left\{\begin{aligned}
\bar{\Omega}_{0}^{*} & =\Omega^{*}\left(X_{0}\right) / \mathscr{I}_{0}, \\
\bar{d} & =d \bmod \mathscr{J}_{0} .
\end{aligned}\right.
$$

Provisional Definition 1.1. The characteristic cohomology $\bar{H}_{0}^{*}$ of the exterior differential system $\mathscr{I}_{0}$ is by definition the cohomology of the complex $\left\{\bar{\Omega}_{0}^{*}, \bar{d}\right\}$,

$$
\bar{H}_{0}^{*}=H\left\{\overline{\mathbf{\Omega}}_{0}^{*}, \bar{d}\right\} \text {. }
$$

Elemenis $\varphi \in \bar{H}_{0}^{*}$ are called characteristic classes of $\mathscr{J}_{0}$. 
We shall explain the subscript " 0 " in Section 1.3 below. The groups $\bar{H}_{0}^{*}$ are only a first approximation to our ultimate objects of interest, which will be defined there and denoted by $\bar{H}^{*}$ without the subscript.

If $f: N \rightarrow X_{0}$ is an integral manifold of $\mathscr{I}_{0}$, there is an induced mapping

$$
f^{*}: \bar{H}_{0}^{*} \rightarrow H_{\mathrm{DR}}^{*}(N) \text {. }
$$

We may think of $f^{*}\left(\bar{H}_{0}^{*}\right)$ as being the cohomology induced on $N$ by virtue of its being a solution to the PDE system. Obvious questions are to "compute" $\bar{H}_{0}^{*}$, to understand how the image of $f^{*}$ varies with $f$, and so forth.

The two extreme cases $\bar{H}_{0}^{n}$ and $\bar{H}_{0}^{0}$ may be fairly easily interpreted. For example, in case $N$ is compact, using our assumption (1), we have that

$$
\bar{H}_{0}^{n}=\Omega^{n}\left(X_{0}\right) / d \Omega^{n-1}\left(X_{0}\right)+\mathscr{J}_{0}^{n},
$$

and each $\lambda \in \Omega^{n}\left(X_{0}\right)$ defines a functional $I_{\lambda}$ on the set of compact integral manifolds by

$$
I_{\lambda}(f)=\int_{N} f^{*}(\lambda) .
$$

Clearly, $I_{\lambda}$ depends only on the class $[\lambda] \in \bar{H}_{0}^{n}$ defined by $\lambda$. The question of how $I_{\lambda}$ varies with $f$ clearly involves the Euler-Lagrange equations of that functional.

Example 1. Pfaffian systems. The understanding of $\bar{H}_{0}^{0}$ is most immediate when $\mathscr{I}_{0}$ is a Pfaffian differential ideal, i.e., it is locally generated as a differential ideal in degree one. Concretely, locally there are linear differential forms $\theta^{1}, \ldots, \theta^{s}$ such that $\mathscr{J}_{0}$ is generated algebraically by $\theta^{1}, \ldots, \theta^{s}$; $d \theta^{1}, \ldots, d \theta^{s}$; we write

$$
\mathscr{I}_{0}=\left\{\theta^{1}, \ldots, \theta^{s}\right\} \text {. }
$$

The derived flag $\mathscr{I}_{0} \supset \mathscr{J}_{0}^{(1)} \supset \mathscr{J}_{0}^{(2)} \supset \cdots$ is by definition the nested sequence of Pfaffian systems defined inductively by $\mathscr{J}_{0}^{(k+1)}=\left\{\theta \in \mathscr{J}_{0}^{(k)}: d \theta \in \mathscr{J}_{0}^{(k)}\right\}$. Then $\mathscr{I}_{0}^{(\infty)}=\bigcap_{k} \mathscr{J}_{0}^{(k)}$ is the largest completely integrable subsystem of $\mathscr{I}_{0}$. Under suitable constant rank assumptions, we may invoke the Frobenius theorem to find local functions $f_{1}, \ldots, f_{r}$ such that

$$
\mathscr{I}_{0}^{(\infty)}=\left\{d f_{1}, \ldots, d f_{r}\right\}
$$

The $\mathbb{R}$-vector space of functions $f$ satisfying $d f \in \mathscr{J}_{0}$ is classically called the space of first integrals of the exterior differential system, denoted here by $F I\left(\mathscr{I}_{0}\right)$. Assumption (1) together with the exact cohomology sequence of $0 \rightarrow$ $\mathscr{I}_{0} \rightarrow \Omega^{*}\left(X_{0}\right) \rightarrow \bar{\Omega}^{*} \rightarrow 0$ gives immediately that locally

$$
\bar{H}_{0}^{0} \cong\left\{g \in \Omega^{0}\left(X_{0}\right): d g \in \mathscr{J}_{0}\right\}=F I\left(\mathscr{I}_{0}\right)
$$

(The assumption that we are working locally is used not only to represent $\mathscr{J}_{0}^{(\infty)}$ globally in the form $\left\{d f_{1}, \ldots, d f_{r}\right\}$ but also to insure that $H_{\mathrm{DR}}^{1}\left(X_{0}\right)=0$.) 
Example 2. Contact manifolds. A more substantial and interesting example is that of a Pfaffian system locally generated by a single 1 -form $\theta_{0}$. With suitable constant rank assumptions, there is an integer $n$ so that

$$
\begin{aligned}
& \theta_{0} \wedge\left(d \theta_{0}\right)^{n} \neq 0, \\
& \theta_{0} \wedge\left(d \theta_{0}\right)^{n+1}=0 .
\end{aligned}
$$

For simplicity, we are going to assume that $\operatorname{dim} X_{0}=2 n+1$. In this case, the ideal $\mathscr{J}_{0}$ generated by $\theta_{0}$ is called a contact ideal and the pair $\left(X_{0}, \mathscr{J}_{0}\right)$ is called a contact manifold. By the Pfaff-Darboux theorem, every point of $X_{0}$ lies in a neighborhood on which there exist local coordinates $\left(x^{1}, \ldots, x^{n}, z\right.$, $\left.y_{1}, \ldots, y_{n}\right)$ in which a non-zero multiple $\theta$ of $\theta_{0}$ has the normal form

$$
\theta=d z-\sum_{i=1}^{n} y_{i} d x^{i}
$$

Maximal integral manifolds of $\theta$ have dimension $n$ and are called Legendre submanifolds of $X_{0}$. Those on which $d x^{1} \wedge \cdots \wedge d x^{n} \neq 0$ are locally of the form

$$
\left(x^{1}, \ldots, x^{n}\right) \rightarrow\left(x^{1}, \ldots, x^{n}, z(x), \frac{\partial z(x)}{\partial x^{1}}, \ldots, \frac{\partial z(x)}{\partial x^{n}}\right) .
$$

We will show that, locally,

$$
\left\{\begin{array}{l}
\bar{H}_{0}^{0} \cong \mathbb{R}, \\
\bar{H}_{0}^{q}=0, \quad q>0, \quad q \neq n, \\
\operatorname{dim} \bar{H}_{0}^{n}=\infty .
\end{array}\right.
$$

On contact submanifolds of the form (2), $\bar{H}_{0}^{n}$ may be thought of as equivalence classes of first order functionals

$$
z(x) \rightarrow \int \lambda\left(x^{1}, \ldots, x^{n}, z(x), \frac{\partial z(x)}{\partial x^{1}}, \ldots, \frac{\partial z(x)}{\partial x^{n}}\right) d x^{1} \wedge \cdots \wedge d x^{n} .
$$

The proof of (2) will be given following a preliminary linear algebra discussion. Suppose that $V$ is a vector space of dimension $2 n+1$ and that we are given

$$
\left\{\begin{array}{l}
\theta \in V \\
\Theta \in \Lambda^{2} V
\end{array}\right.
$$

satisfying the conditions that (i) $\Theta$ is well defined up to adding multiples $\alpha \wedge \theta$ of $\theta$, and (ii) $\theta \wedge \Theta^{n} \neq 0$. (We are obviously thinking of $V$ as being a typical $T_{p}^{*} X_{0}$.) Set $\bar{V}=V / \mathbb{R} \theta$ and let $\bar{\Theta} \in \Lambda^{2} \bar{V}$ be induced by $\Theta$. Then $\bar{\Theta}$ is a non-degenerate 2-form on $\bar{V}$ and it is well known that there is a so-called "primitive" or "Lefschetz" decomposition of $\Lambda^{*} \bar{V}$. Recall that this comes about as follows: First, one shows that

$$
\bar{\Theta}^{k}: \Lambda^{n-k} \bar{V} \stackrel{\sim}{\rightarrow} \Lambda^{n+k} \bar{V}
$$

is an isomorphism for $1 \leqq k \leqq n$. Next, if for $k \geqq 0$ one defines the primitive space by

$$
\bar{P}^{n-k}=\operatorname{ker}\left\{\bar{\Theta}^{k+1}: \Lambda^{n-k} \bar{V} \rightarrow \Lambda^{n+k+2} \bar{V}\right\},
$$


then there is a Lefschetz decomposition

$$
\Lambda^{n-k} \bar{V}=\bar{P}^{n-k} \oplus \bar{\Theta} \cdot \bar{P}^{n-k-2} \oplus \bar{\Theta}^{2} \cdot \bar{P}^{n-k-4} \oplus \cdots
$$

If we now let $I \subset \Lambda^{*} V$ be the homogeneous ideal generated by $\theta$ and $\theta$, it follows from (i) that

$$
I^{n+k}=\Lambda^{n+k} V, \quad k \geqq 1
$$

Moreover, from (ii) we see that for $k \geqq 0$ each $\psi \in \Lambda^{n-k} V$ has an expression

$$
\psi=\theta \wedge \alpha+\beta_{0}+\Theta \wedge \beta_{1}+\Theta^{2} \wedge \beta_{2}+\cdots
$$

where the $\beta_{i}$ are unique modulo $\theta$ and satisfy $\theta^{k+2 i+1} \wedge \beta_{i} \equiv 0 \bmod \theta$.

We now apply this discussion where $V$ is a typical $T_{p}^{*} X_{0}$, the 1 -form $\theta$ is given by $d z-\sum y_{i} d x^{i}$, and $\Theta=d \theta=-\sum d y_{i} \wedge d x_{i}$. We shall denote by

$$
P^{n-k} \subset \Omega^{n-k}\left(X_{0}\right), \quad k \geqq 0,
$$

the space of forms that are primitive modulo $\theta$, i.e., that satisfy

$$
\Theta^{k+1} \wedge \alpha \equiv 0 \bmod \theta
$$

Using (iv), we have a decomposition of all forms in $\Omega^{n-k}\left(X_{0}\right)$ in terms of multiples of $\theta$ and primitive forms.

From (iii) it follows that $\mathscr{J}_{0}^{n+k}=\Omega^{n+k}\left(X_{0}\right)$ for all $k \geqq 1$ which clearly implies that $\Omega_{0}^{n+k}=0$ for all $k \geqq 1$ (cf. (1) above). This trivially implies that $\bar{H}_{0}^{q}=0$ for $q \geqq n+1$.

From the exact cohomology sequence of

$$
0 \rightarrow \mathscr{I}_{0} \rightarrow \Omega^{*}\left(X_{0}\right) \rightarrow \Omega_{0}^{*} \rightarrow 0,
$$

and remembering that we are working locally (so that $H^{q}\left(X_{0}\right)=0$ for $q>0$ ), we see that the coboundary map induces isomorphisms

$$
\delta: \bar{H}_{0}^{q} \stackrel{\sim}{\rightarrow} H^{q+1}\left(\mathscr{J}_{0}\right), \quad q \geqq 1 .
$$

Using this we will first prove that $\bar{H}_{0}^{q}=0$ for $0<q<n$ by showing that

$$
H^{p}\left(\mathscr{I}_{0}\right)=0, \quad 0 \leqq p \leqq n .
$$

Let

$$
\psi=\theta \wedge \alpha+\Theta \wedge \beta \in \mathscr{I}_{0}^{p}
$$

be closed. Then $\alpha, d \beta \in \Omega^{p-1}\left(X_{0}\right)$ and

$$
\begin{aligned}
0 & =\boldsymbol{\theta} \wedge(\alpha+d \beta)-\theta \wedge d \alpha \\
& \Longrightarrow \quad \alpha+d \beta=\theta \wedge \gamma \quad \text { by }(\mathrm{i}) \\
& \Longrightarrow \psi=d(\theta \wedge \beta)
\end{aligned}
$$

which gives (4). 
We now turn to the most interesting group $\bar{H}_{0}^{n}$. We will show that a closed form $\eta \in \mathscr{J}_{0}^{n+1}\left(=\Omega^{n+1}\left(X_{0}\right)\right)$ has an expression

$$
\left\{\begin{array}{l}
\eta \equiv \theta \wedge \pi \bmod d \mathscr{J}_{0}^{n}, \\
\pi \in P^{n}
\end{array}\right.
$$

where $\pi$ is unique $\bmod \theta$ and depends only on the class $[\eta] \in H^{n+1}\left(\mathscr{J}_{0}\right)$ of $\eta$. Moreover, given $\pi \in P^{n}$ we have by definition that

$$
\Theta \wedge \pi=\theta \wedge \gamma \text {. }
$$

Replacing $\pi$ by $\pi+\theta \wedge \alpha$ gives $\gamma \rightarrow \gamma+\Theta \wedge \alpha$. Thus, we may uniquely determine $\pi$ by further requiring that $\Theta \wedge \pi=0$. The condition that $\eta=\theta \wedge \pi$ be closed is then that

$$
d \pi \wedge \theta=0
$$

Thus

$$
\bar{H}_{0}^{n} \cong H^{n+1}\left(\mathscr{I}_{0}\right) \cong\left\{\pi \in P^{n} \bmod \theta: d \pi \wedge \theta=0\right\}
$$

is naturally represented as the sections of a vector bundle (actually a subbundle of the differential forms on $X_{0}$ ) satisfying a linear differential equation. (In the involutive case, this sort of "subspace" rather than "quotient" representation of $\bar{H}_{0}^{n}$ will be a characteristic feature of the first non-vanishing $\bar{H}^{q}$ with $q>0$, once these groups have been defined.)

Turning to the proof of (5), write

$$
\eta=\theta \wedge \alpha+\Theta \wedge \beta
$$

Then, as before, $d \Theta=0$ gives that

$$
\begin{aligned}
& \Theta \wedge(\alpha+d \beta) \equiv 0 \bmod \theta \\
& \Longrightarrow \quad \alpha+d \beta=\pi+\theta \wedge \gamma
\end{aligned}
$$

where $\pi \in P^{n}$ is primitive $\bmod \theta$

$$
\Longrightarrow \eta=d(\theta \wedge \beta)+\theta \wedge \pi
$$

To show uniqueness we suppose that we have

$$
d \xi=\theta \wedge \pi
$$

for some $\xi=\theta \wedge \rho+\Theta \wedge \sigma \in \mathscr{I}^{n}$. This gives

$$
\begin{aligned}
\theta \wedge & (\rho+d \sigma)-\theta \wedge d \rho=\theta \wedge \pi \\
& \Longrightarrow \quad \rho+d \sigma=\theta \wedge \gamma \quad \text { for some } \gamma \\
& \Longrightarrow \xi=d(\theta \wedge \sigma) \\
& \Longrightarrow \quad \theta \wedge \pi=0 .
\end{aligned}
$$

Thus, $\pi$ is unique $\bmod \theta$ and depends only on the class $[\eta] \in H^{n+1}\left(\mathscr{J}_{0}\right)$, as we wished to show.

Using the isomorphism (3) we have

$$
\delta: \bar{H}_{0}^{n} \stackrel{\sim}{\rightarrow}\left\{\pi \in P^{n} \bmod \theta: d \pi \wedge \theta=0\right\} .
$$


As we shall now explain, the mapping $\delta$ is a second order linear differential operator which may be interpreted as the Euler-Lagrange equations associated to the functional defined by $\bar{\psi} \in \bar{\Omega}_{0}^{n}$.

Given $\bar{\psi}$ we seek a lifting $\psi \in \Omega^{n}\left(X_{0}\right)$ such that

$$
d \psi=\theta \wedge \pi
$$

where $\pi \in P^{n}$. If $\psi^{\prime}$ is any lifting of $\bar{\psi}$, then as in (7) we have

$$
\begin{aligned}
d \psi^{\prime} & =\theta \wedge \pi+d(\theta \wedge \beta) \\
& \Longrightarrow \quad d\left(\psi^{\prime}-\theta \wedge \beta\right)=\theta \wedge \pi .
\end{aligned}
$$

Taking $\psi=\psi^{\prime}-\theta \wedge \beta$ gives (8). We note that each of the mappings

$$
\left\{\begin{array}{l}
\bar{\psi} \rightarrow \psi \\
\psi \rightarrow \pi
\end{array}\right.
$$

are first-order linear differential operators, so that $\bar{\psi} \rightarrow \pi$ is second order as claimed.

In Section 3.3 we will further discuss the infinite prolongation of this example, in effect giving a resolution of $\bar{H}^{n}$ by a complex of canonical linear, first-order differential operators.

Example 3. Symplectic manifolds. Now let us suppose that $\operatorname{dim} X_{0}=2 n$ and that $\Theta$ is a closed 2-form on $X_{0}$ which satisfies the condition that $\Theta^{n}$ is nowhere vanishing, i.e., that $\Theta$ defines a symplectic structure on $X_{0}$. We shall consider the differential ideal $\mathscr{J}_{0}$ generated by the non-degenerate 2-form $\Theta$. Also, for simplicity, we shall exclude the trivial case $n=1$ and henceforth assume that $n>1$. By the Pfaff-Darboux theorem, in suitable local coordinates $\left(x^{1}, \ldots, x^{n}, \xi_{1}, \ldots, \xi_{n}\right)$ on $X_{0}$ we have

$$
\Theta=d \theta=\sum_{i=1}^{n} d \xi_{i} \wedge d x^{i}
$$

The maximal integral manifolds of $\Theta$ have dimension $n$ and are generally referred to as Lagrangian submanifolds of $X_{0}$. Those on which $d x^{1} \wedge \cdots \wedge d x^{n} \neq$ 0 are locally of the form

$$
\left(x^{1}, \ldots, x^{n}\right) \rightarrow\left(x^{1}, \ldots, x^{n}, \frac{\partial S(x)}{\partial x^{1}}, \ldots, \frac{\partial S(x)}{\partial x^{n}}\right)
$$

where $S(x)$ is an arbitrary smooth function. We will show that, locally,

$$
\begin{aligned}
& \bar{H}_{0}^{q}=0 \text { for } q \neq 0,1, \text { or } n, \\
& \bar{H}_{0}^{0} \cong \mathbb{R}, \\
& \bar{H}_{0}^{1} \cong \mathbb{R}, \\
& \operatorname{dim} \bar{H}_{0}^{n}=\infty .
\end{aligned}
$$

(The case $n=1$ is somewhat analogous but requires a separate discussion.) The proof is very similar to the contact manifold case and will only be sketched. 
From $\Theta^{k}: \Omega^{n-k}\left(X_{0}\right) \stackrel{\sim}{\rightarrow} \Omega^{n+k}\left(X_{0}\right), k \geqq 1$, we have $\mathscr{I}_{0}^{n+k}=\Omega^{n+k}\left(X_{0}\right)$ for $k \geqq 1$ and as a trivial consequence $\bar{H}_{0}^{q}=0$ for $q>n$.

As in (3) we have isomorphisms

$$
\delta: \bar{H}_{0}^{q}: \stackrel{\sim}{\rightarrow} H^{q+1}\left(\mathscr{F}_{0}\right)
$$

for $q>0$. If

$$
\psi=\Theta \wedge \alpha \in \mathscr{F}_{0}^{p}
$$

is closed, then

$$
0=d \psi=\Theta \wedge d \alpha .
$$

Since $d \alpha \in \Omega^{p-1}\left(X_{0}\right)$ we infer that $d \alpha=0$ if $p \leqq n$. Thus, if $3 \leqq p \leqq n$, then we may write locally $\alpha=d \beta$, which gives $\psi=d\left(\boldsymbol{\Theta}_{\wedge} \beta\right)$, which implies that $0=H^{p}\left(\mathscr{I}_{0}\right) \cong \bar{H}_{0}^{p-1}$. If $p=2$, then $\alpha=C$ is a constant and $\psi=C \Theta$, from which we infer that $\bar{H}_{0}^{1} \cong \mathbb{R}$. Finally, if $p=n+1$, then $d \alpha=\pi$ is a closed, primitive $n$-form. It is easily checked that $\pi$ depends only on the class $[\psi] \in H^{n+1}\left(\mathscr{J}_{0}\right)$. This gives the identification

$$
\delta: \bar{H}_{0}^{n} \stackrel{\sim}{\rightarrow} \text { closed, primitive } n \text {-forms } \text {. }
$$

Again, $\bar{H}_{0}^{n}$ is represented as the kernel of a canonical linear differential operator, modulo nothing.

Remark. If locally we choose a 1-form $\alpha$ with $d \alpha=\boldsymbol{\Theta}$, then the most general solution of $d \theta=\Theta$ is $\theta=\alpha+d f$ for an arbitrary function $f$. This suggests that we consider the "universal" solution to this problem, which is obtained by taking

$$
Y=X_{0} \times \mathbb{R}
$$

where $\mathbb{R}$ has coordinate $u$ and setting

$$
\theta=d u-\alpha
$$

on $Y$. If $\mathscr{J} \subset \Omega^{*}(Y)$ is the exterior differential system generated by $\theta$, then the projection $\pi: Y \rightarrow X_{0}$ induces mappings

$$
\begin{array}{ccc}
\Omega^{*}\left(X_{0}\right) & \hookrightarrow & \Omega^{*}(Y) \\
\cup & & \cup \\
\mathscr{J}_{0} & \hookrightarrow & \mathscr{J}
\end{array}
$$

which give

$$
\pi^{*}: \bar{H}^{q}\left(X_{0}\right) \longrightarrow \bar{H}_{0}^{q}(Y)
$$

This mapping $\pi^{*}$ is an isomorphism for $q \neq 0,1$, or $n$, while $\pi^{*}$ kills $\bar{H}_{0}^{1}\left(X_{0}\right)$.

This process of adjoining a "primitive" or potential (think of $u$ as $\int \alpha$ on integral manifolds of $\theta=0$ ) will reappear and be formalized in Part II in connection with conservation laws of certain specific parabolic systems (such as Burger's equation).

We will conclude this section with a discussion of two related examples. 
Example 4. Complex manifolds. Let $X_{0}$ be a complex manifold, with $\Omega^{p, q}\left(X_{0}\right)$ denoting the space of smooth $(p, q)$-forms on $X_{0}$. We let $\mathscr{I}_{0}$ be the differential ideal generated by the 2 -forms

$$
\theta=\alpha+\bar{\alpha}, \quad \alpha \in \Omega^{2,0}\left(X_{0}\right) .
$$

Clearly, $\mathscr{J}_{0}^{1}=0$ and $\mathscr{F}_{0}^{q}=\Omega^{q}\left(X_{0}\right)$ for $q \geqq 3$. Integral manifolds of $\mathscr{I}_{0}$ are thus of (real) dimension at most two, and those of dimension two are holomorphic curves in $X_{0}$. The first interesting characteristic cohomology group is $\bar{H}_{0}^{1}$, and denoting by $\Omega_{c}^{p, 0}\left(X_{0}\right)$ the closed (and therefore holomorphic) $(p, 0)$ forms, we shall show that

$$
\bar{H}_{0}^{1} \cong \Omega_{c}^{2,0}\left(X_{0}\right)
$$

Proof. A class in $\bar{H}_{0}^{1}$ is represented by

$$
\eta=\eta^{\prime}+\bar{\eta}^{\prime}, \quad \eta^{\prime} \in \Omega^{1,0}\left(X_{0}\right),
$$

which satisfies

$$
\bar{\partial} \eta^{\prime}+\partial \bar{\eta}^{\prime}=0 .
$$

Then

satisfies

$$
\pi=\partial \eta^{\prime} \in \Omega^{2,0}\left(X_{0}\right)
$$

$$
\bar{\partial} \pi=-\partial \bar{\partial} \eta^{\prime}=\partial^{2} \bar{\eta}^{\prime}=0 .
$$

It is easily seen that $\pi$ depends only on the class of $\eta \in \bar{H}_{0}^{1}$, and that any $\pi \in \Omega_{c}^{2,0}\left(X_{0}\right)$ arises from a class in $\bar{H}_{0}^{1}$. Q.E.D.

Again we encounter the phenomenon that the first non-vanishing characteristic cohomology group is isomorphic to the kernel of a canonical linear differential operator, modulo nothing.

As will be made clear when we discuss conservation laws, in this example the group $\bar{H}_{0}^{1}$ can be interpreted as the space of independent conservation laws for this system. Note that when $X_{0}=\mathbb{C}^{n}$, this space is of infinite dimension. This is perhaps the simplest non-trivial example of an exterior differential system with an infinite number of independent local conservation laws. Ultimately these are rooted in the Cauchy integral formulas

$$
\frac{d}{d t}\left(\frac{1}{2 \pi i} \int_{|z|=t} z^{k} f(z) d z\right)=0
$$

for holomorphic functions in the complex plane.

Example 5. Associative manifolds. Our last example is based on Bryant [3] and especially on work of Harvey-Lawson [7] on calibrated geometries. We denote by $\mathbb{O}$ the octonions with standard basis $e_{0}, e_{1}, \ldots, e_{8}$ where $e_{0}$ is the unit. We then let $\mathbb{R}^{7}=\operatorname{Im} \mathbb{0}$ be the imaginary octarians with the standard metric 
having $e_{1}, \ldots, e_{7}$ as an orthonormal basis. Using $x^{1}, \ldots, x^{7}$ as dual linear coordinates on $\mathbb{R}^{7}$ and setting

$$
d x^{i j k}=d x^{i} \wedge d x^{j} \wedge d x^{k},
$$

we consider the 3-form

$$
\varphi=d x^{123}+d x^{145}+d x^{167}+d x^{246}-d x^{257}-d x^{347}-d x^{356}
$$

which gives the multiplication table of $\operatorname{Im} \mathbb{O}$ in the following manner:

(i) $e_{i}^{2}=-e_{0}$,

(ii) for $i, j$ distinct

$$
e_{i} \cdot e_{j}+e_{j} \cdot e_{i}=0
$$

(iii) for $i, j k$ distinct we have

$$
e_{i} \cdot e_{j}=e_{k}
$$

if and only if $d x^{i j k}$ appears with a $+\operatorname{sign}$ in $\varphi$.

The form $\varphi$ gives a calibration in the sense that for any vectors $v_{1}, v_{2}, v_{3}$

$$
\left|\varphi\left(v_{1}, v_{2}, v_{3}\right)\right| \leqq\left|v_{1} \wedge v_{2} \wedge v_{3}\right|
$$

with equality holding if and only if $v_{1}, v_{2}, v_{3}$ span a 3-plane that is closed under multiplication (these are called associative 3-planes). We note the analogy to Kähler geometry, where for

$$
\psi=\frac{\sqrt{-1}}{2} \sum_{i=1}^{n} d z^{i} \wedge d \bar{z}^{i}=\sum_{i=1}^{n} d x^{i} \wedge d y^{i}
$$

in $\mathbb{C}^{n}$ with coordinates $z^{i}=x^{i}+\sqrt{-1} y^{i}$, we have Wirtinger's inequality

$$
\left|\psi\left(v_{1}, v_{2}\right)\right| \leqq\left|v_{1} \wedge v_{2}\right|
$$

with equality if and only if $v_{1}$ and $v_{2}$ are either linearly dependent or span a complex line. By further analogy, we will define an exterior differential system on $\mathbb{R}^{7}$ whose 3 -dimensional integral elements are exactly the associative 3 planes.

For this we set

$$
\left.\Theta_{i}=e_{i}\right\lrcorner * \varphi
$$

and recall from the references given above that (10) may be completed into an equality by virtue of the relation

$$
\left|\varphi\left(v_{1}, v_{2}, v_{3}\right)\right|^{2}+\sum_{i=1}^{7}\left|\Theta_{i}\left(v_{1}, v_{2}, v_{3}\right)\right|^{2}=\left|v_{1} \wedge v_{2} \wedge v_{3}\right|^{2}
$$

Thus the exterior equations

$$
\Theta_{i}=0
$$

generate an exterior differential system $\mathscr{I}_{0}$ on $\mathbb{R}^{7}$ whose 3 -dimensional integral elements are exactly the associative 3-planes. We note that

$$
\mathscr{F}_{0}^{q}=0, \quad q=0,1,2
$$


and it may be shown that

$$
\mathcal{F}_{0}^{q}=\Omega^{q}\left(\mathbb{R}^{7}\right), \quad q \geqq 4 .
$$

Thus the interesting characteristic cohomology group is $\bar{H}_{0}^{2}$, and we will show that

$$
\operatorname{dim} \bar{H}_{0}^{2}=21 .
$$

Proof. It is clear that

$$
\bar{H}_{0}^{2} \cong \text { closed forms in } \mathscr{I}_{0}^{3} .
$$

Now any form in $\mathscr{J}_{0}^{3}$ is

$$
\psi=X\lrcorner * \varphi
$$

for some vector field $X$, and from

$$
0=d \psi=d(X\lrcorner * \varphi)=\mathscr{L}_{X}(* \varphi),
$$

we see that $\exp (t X)$ preserves the form $* \varphi$. We will show that

(i) the mapping $\varphi \rightarrow * \varphi$ is $2: 1$, so that $\exp (t X)$ preserves $\varphi$; and

(ii) $\varphi$ determines the metric, so that $X$ is an infinitesimal Euclidean motion.

This proves already that $\operatorname{dim} \bar{H}_{0}^{2}<\infty$, and the fact that $\operatorname{dim} \bar{H}_{0}^{2}=21$ will depend on knowing that the symmetry group of $\varphi$ is the exceptional group $G_{2}$, which has dimension 14 .

Let $V$ be a 7-dimensional real vector space and $\varphi \in \Lambda^{3} V^{*}$. We note that this is one of the very few cases (other than the obvious ones) where $G L(V)$ acting on $\Lambda^{q} V^{*}$ has an open orbit. For $\varphi$ as above we define a symmetric bilinear map

$$
b_{\varphi}: V \times V \longrightarrow \Lambda^{7} V^{*}
$$

by

$$
\left.\left.b_{\varphi}(v, w)=\frac{1}{6}(v\lrcorner \varphi\right) \wedge(w\lrcorner \varphi\right) \wedge \varphi .
$$

We say that $\varphi$ is non-degenerate if $b_{\varphi}$ is non-singular, and observe that the $\varphi$ given above has this property. To convert $b_{\varphi}$ into a metric we need to single out a canonical volume form, which we now do by singling out unimodular bases for $V$. If $v_{1}, \ldots, v_{7}$ is any basis we set

$$
B_{\varphi}^{v}\left(v_{i}, v_{j}\right)=b_{\varphi}\left(v_{i}, v_{j}\right)\left(v_{1}, \ldots, v_{7}\right) \text {. }
$$

If $A \in G L_{7}(\mathbb{R})$ transforms $v_{i}$ to $\tilde{v}_{i}$, then a calculation shows that

$$
\operatorname{det}_{i, j}\left\|B_{\varphi}^{\tilde{v}}\left(v_{i}, v_{j}\right)\right\|=(\operatorname{det} A)^{9} \operatorname{det}_{i, j}\left\|B_{\varphi}^{v}\left(v_{i}, v_{j}\right)\right\| .
$$

For non-degenerate $\varphi$, we will say that a basis $v_{1}, \ldots, v_{7}$ of $V$ is $\varphi$-unimodular in case $\operatorname{det}_{i, j}\left\|B_{\varphi}^{v}\left(v_{i}, v_{j}\right)\right\|=1$. In this way a non-degenerate $\varphi$ gives a metric on $\mathbb{R}^{7}$. For the particular $\varphi$ given above, $B_{\varphi}^{e}\left(e_{i}, e_{j}\right)=\delta_{i j}$ is the standard metric. 
The fact that the mapping $\varphi \rightarrow * \varphi$ is one-to-one near our particular $\varphi$ (which is all that we need) is easily shown by computing the differential of the *-mapping at $\varphi$. It follows that any 1-parameter subgroup of diffeomorphisms of $\mathbb{R}^{7}$ which preserves $* \varphi$ must also preserve $\varphi$ and the standard flat metric. Since the group of diffeomorphisms which preserve $\varphi$ clearly contains the translations, it follows that this group must be the semi-direct product of the translations with the subgroup of those rotations about the origin which preserve $\varphi$.

At this stage we have shown that

$$
X=T+L
$$

where $T$ is a translation vector field (corresponding to constant linear combinations of the $\left.\Theta_{i}\right)$ and $L \in \mathfrak{g l}\left(\mathbb{R}^{7}\right)$ preserves $\varphi$. But since $\varphi$ determines the multiplication in $\operatorname{Im} \mathbb{O}$ it follows that $L$ induces an infinitesimal automorphism of $\mathbb{O}$, and it is then known that $L$ must lie in $\mathfrak{g}_{2} \subset \mathfrak{g l}\left(\mathbb{R}^{7}\right)$ where $\mathfrak{g}_{2}$ is the Lie algebra of $G_{2}$ embedded in $\mathfrak{g l}\left(\mathbb{R}^{7}\right)$ by the standard representation $G_{2} \rightarrow G L(\operatorname{Im} \mathbb{O})$.

Remark. We have shown that for any connected open set $U \subset \mathbb{R}^{7}$ the characteristic cohomology $\bar{H}_{0}^{2}(U)$ is a 21-dimensional vector space. In a sense this example therefore resembles more the symplectic example above than the complex manifold example. However, in Section 6 below we will see that the differences are more important than the similarities.

1.2. The exterior differential system associated to a partial differential equation; symmetries. It is well known that any partial differential equation system-PDE system for short-may be canonically rewritten as an exterior differential system in such a way that the solutions to the PDE system give integral manifolds of the exterior differential system. The converse is also true provided that we work locally and impose a transversality condition on integral manifolds. However, the exterior differential system formulation gives a coordinate free method of studying the system; moreover, the larger group of symmetries of the exterior differential system will allow us to do calculations of examples not yet possible in the coordinate formulation. In this section we will briefly review this construction. For simplicity of exposition we will restrict ourselves to the case of a first-order PDE system, remarking that similar considerations apply to the general situation.

The most classical formulation of a PDE system is to give a submanifold $R$, satisfying suitable conditions to be specified below, of the manifold $J^{1}(N, Y)$ of 1 -jets of smooth mappings from a manifold $N$ to a manifold $Y$. In this formulation, the symmetries are the diffeomorphisms of $R$ that preserve the two coordinate projections $R \rightarrow N$ and $R \rightarrow Y$. Concretely, let $x^{1}, \ldots, x^{n}$ and $u^{1}, \ldots, u^{s}$ be local coordinates on $N$ and $Y$, respectively. Then, using the ranges of indices

$$
1 \leqq i, j \leqq n, \quad 1 \leqq \alpha, \beta \leqq s,
$$

there are induced local coordinates $\left\{x^{i}, u^{\alpha}, p_{i}^{\alpha}\right\}$ on $J^{1}(N, Y)$. Symmetries 
are induced by diffeomorphic changes of independent and dependent variables

$$
\left\{\begin{array}{l}
x^{\prime i}=x^{\prime i}(x), \\
u^{\prime \alpha}=u^{\prime \alpha}(u), \\
p_{i}^{\prime \alpha}=\partial u^{\prime \alpha} / \partial u^{\beta} p_{j}^{\beta} \partial x^{j} / \partial x^{\prime i} .
\end{array}\right.
$$

Each mapping $f: N \rightarrow Y$ given locally by $x^{i} \rightarrow u^{\alpha}(x)$ has a canonical lifting $j_{1}(f) \subset J^{1}(N, Y)$ given in coordinates by $\left\{x^{i}, u^{\alpha}(x), \partial u^{\alpha} / \partial x^{i}\right\}$. That is, $J^{1}(N, Y)$ is just the vector bundle $\operatorname{Hom}(T N, T Y)$ lying over $N \times Y$, and associated to a mapping $f: N \rightarrow Y$ is first of all the graph $\Gamma_{f} \subset N \times Y$ of $f$, and then lying over this is the graph $j_{1}(f) \subset J^{1}(N, Y)$ of the differential of $f$.

A submanifold $R \subset J^{1}(N, Y)$ may be thought of as imposing constraints on the differentials of maps from $N$ to $Y$; i.e., as a PDE system. In coordinates, $R$ is given locally by equations

$$
F^{\lambda}\left(x^{i}, u^{\alpha}, p_{i}^{\alpha}\right)=0
$$

to which corresponds the PDE system

$$
F^{\lambda}\left(x^{i}, u^{\alpha}(x), \partial u^{\alpha}(x) / \partial x^{i}\right)=0 .
$$

We assume that the equations (2) define a submanifold $R \subset J^{1}(N, Y)$ and that the projection $R \rightarrow N$ is a submersion. As explained above, the group Diff $N \times$ Diff $Y$ acts as a group of diffeomorphisms of $J^{1}(N, Y)$, and the subgroup preserving $R$ is by definition the classical symmetry group of the PDE system (3).

A generalization arises by considering a fibered manifold $Z \rightarrow N$ and defining a PDE system to be given by a submanifold $R \subset J^{1}(N, Z)$ where $J^{1}(N, Z)$ is the manifold of 1 -jets of smooth sections of the fibered manifold. By definition of a fibered manifold, the projection $Z \rightarrow N$ is a submersion, and locally on $Z$ there are product coordinates $\left(x^{i}, u^{\alpha}\right)$ such that $Z \rightarrow N$ is given by $\left(x^{i}, u^{\alpha}\right) \rightarrow\left(x^{i}\right)$. Then there are induced local coordinates $\left(x^{i}, u^{\alpha}, p_{i}^{\alpha}\right)$ on $J^{1}(N, Z)$, and $R$ given by (2) corresponds to the PDE system (3).

The difference between these two constructions is that in the fibered manifold context the symmetry group is enlarged to include gauge transformations. That is, the automorphism group of $J^{1}(N, Z)$ is induced from the automorphisms of the fibered manifold, defined to be the fiber-preserving diffeomorphisms of $Z$ given in local coordinates by

$$
\left\{\begin{array}{l}
x^{\prime i}=x^{\prime i}(x), \\
u^{\prime \alpha}=u^{\prime \alpha}(u, x), \\
p_{i}^{\prime \alpha}=\left(\left(\partial u^{\prime \alpha} / \partial u^{\beta}\right) p_{j}^{\beta}+\partial u^{\prime \alpha} / \partial x^{j}\right) \partial x^{j} / \partial x^{\prime \alpha} .
\end{array}\right.
$$

The enlarged symmetry group of $R$ now consists of all transformations (4) which preserve $R$. 
Associated canonically to either a classical PDE system or to a PDE system imposed on sections of a fibered manifold is an exterior differential system. The underlying manifold is $R$, and the exterior differential system is the restriction to $R$ of the contact system defined on either of $J^{1}(N, Y)$ or $J^{1}(N, Z)$. The contact system is the Pfaffian system locally generated by the 1 -forms

$$
\theta^{\alpha}=d u^{\alpha}-p_{i}^{\alpha} d x^{i}
$$

Of course, the contact system may be described in a coordinate-free manner which makes apparent the invariance under the corresponding symmetry group, but we shall not do this here. An integral manifold of this system on which the transversality condition $d x^{1} \wedge \cdots \wedge d x^{n} \neq 0$ is satisfied is locally a graph

$$
\left(x^{i}\right) \rightarrow\left(x^{i}, u^{\alpha}(x), p_{i}^{\alpha}(x)\right)
$$

and the vanishing of the contact forms when restricted to this submanifold implies that

$$
p_{i}^{\alpha}(x)=\partial u^{\alpha}(x) / \partial x^{i}
$$

Restricting to $R$ therefore gives an exterior differential system $\mathscr{I}$ whose integral manifolds satisfying a transversality condition are locally in one-to-one correspondence with solutions to the PDE system.

However, by definition the symmetries of $\mathscr{I}$ are the diffeomorphisms of $R$ that preserve $\mathscr{I}$. The group $\operatorname{Aut}(\mathscr{I})$ of these symmetries certainly contains the gauge group described locally by the transformations (4) preserving $R$, but $\operatorname{Aut}(\mathscr{I})$ may be strictly bigger than this gauge group. The constructions in which we will be interested in this paper are all invariant under this larger group.

Moreover, regarding two PDE $R_{1}$ and $R_{2}$ as the same if there is a diffeomorphism $R_{1} \rightarrow R_{2}$ which identifies the ideals $\mathscr{I}_{1}$ and $\mathscr{I}_{2}$ is a natural notion of equivalence which properly generalizes gauge equivalence. For example, as we shall eventually see, the following equations, though not gauge equivalent to linear equations, lead to manifolds $R$ with ideals $\mathscr{f}$ which are (globally) equivalent to those generated by linear PDE:

$$
\begin{aligned}
& u_{t}=\frac{a u_{x x}+b}{c u_{x x}+d}, \quad a d-b c=-1, \\
& u_{x x} u_{y y}-u_{x y}^{2}=-1, \\
& u_{x x}-k^{2}\left(u_{y}\right) u_{y y}=0, \quad k\left(u_{y}\right) \neq 0,
\end{aligned}
$$

and, more generally,

$$
u_{t}+f(u) u_{x}=0
$$

where $u \in \mathbb{R}^{2}$ and the matrix $f(u)$ has everywhere distinct real eigenvalues.

Before going on we would like to mention one piece of terminology. Namely, when $R$ is all of $J^{1}(N, Y)$ or $J^{1}(N, Z)$-i.e., when there are no equations (2)-we shall say that we are in the unconstrained case. In this case it is a classical result due to Lie and Bäcklund that all symmetries preserving the contact system are in fact gauge transformations (4) when $s>1$. When $s=1$ the 
transformations preserving $\mathscr{I}$ are only required to preserve the single contact form $\theta=d u-p_{i} d x^{i}$ up to a scalar factor, and it is well known that this group is strictly larger than (4). Such symmetries may not preserve the above transversality condition and are analogous to the contact transformations of classical mechanics.

We remarked above that Aut $(\mathscr{J})$ may be strictly larger than the gauge group. A discussion to the effect that this is the exception rather than the rule is given in Vinogradov [14]. Vinogradov's claim is analogous to the statement that an isometry of a generic submanifold of Euclidean space is induced by a Euclidean motion. For our eventual purposes we are interested in non-generic exterior differential systems; e.g., those having a large number of conservation laws. For these the distinction may be more significant. More importantly, we shall use the method of frame adaptation (equivalence method of E. Cartan) to study in practice the conditions imposed on a particular class of exterior differential systems by the requirement that there be conservation laws. This method consists in successively normalizing the invariants of the exterior differential system and refining the frame adaptations-i.e., reducing the structure group-accordingly. These frame adaptations will be made without reference to a particular set of independent variables and are therefore reflective of Aut $(\mathscr{I})$ rather than the smaller group of gauge transformations. The freedom to make such frame adaptations will be absolutely essential for the application of the general theory.

1.3. The prolongation tower. Given an exterior differential system $\mathscr{I}_{0}$ on a manifold, we have introduced the characteristic cohomology groups $\bar{H}_{0}^{q}$ and remarked that, for example, $\bar{H}_{0}^{n}$ may be interpreted as functionals on integral manifolds. Natural questions that may arise are: "What are the Euler-Lagrange equations of such a functional?", and the inverse problem "When is a given PDE system the Euler-Lagrange equations of a functional?" It is well known that, in general, the Euler-Lagrange equations of a functional $\int \lambda\left(x, u, \partial u, \ldots, \partial^{k} u\right) d x$ will have order $2 k$, but in a number of very interesting cases (e.g., the Einstein equations) the order may be less. Thus, in the inverse problem we should not specify the order of derivative in the unknown functional, i.e., we should work on the manifold of jets of arbitrary order.

Somewhat more substantively, we shall see that for the exterior differential system arising from a determined PDE system, the group $\bar{H}_{0}^{n-1}$ is essentially the space of conservation laws for the PDE system. As famously illustrated by the KdV equation, these conservation laws may occur at arbitrarily high jet levels. These considerations suggest that we extend the original problem by allowing jets of arbitrarily high order, a process known as prolongation. Thus, given $\mathscr{J}_{0}$ on $X_{0}$ under certain regularity assumptions we will pass to the infinite prolongation $\mathscr{I}$ on an infinite-dimensional manifold $X$, thereby encapsulating behavior of arbitrarily high order.

More formally, let $G_{n}\left(T X_{0}\right) \rightarrow X_{0}$ denote the bundle whose fiber over a point $p \in X_{0}$ is the Grassmannian $G_{n}\left(T_{p} X_{0}\right)$ of $n$-planes in the tangent space $T_{p} X_{0}$. Over $G_{n}\left(T_{p} X_{0}\right)$ there is a canonical exterior differential system denoted by $\mathscr{L}_{1}$ whose integral manifolds are just the canonical lifts (Gauss mappings) 
of immersions $f: N \rightarrow X_{0}:$

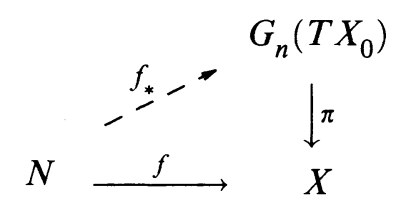

Given $E_{0} \in G_{n}\left(T X_{0}\right)$ we may choose local coordinates $x^{1}, \ldots, x^{n}, u^{1}, \ldots, u^{s}$ on $X_{0}$ such that $E_{0}$ is given by $d u^{\alpha}=0$. A neighborhood of $E_{0}$ in $G_{n}\left(T X_{0}\right)$ then has local coordinates $\left(x^{i}, u^{\alpha}, p_{i}^{\alpha}\right)$ where the corresponding $n$-plane is given by

$$
\theta^{\alpha}=d u^{\alpha}-p_{i}^{\alpha} d x^{i}=0 .
$$

More intrinsically, for an $n$-plane $E \in G_{n}\left(T_{p} X_{0}\right)$ the subspace

$$
\pi^{*}\left(E^{\perp}\right) \subset \pi^{*}\left(T_{p}^{*} X_{0}\right) \subset T_{E}^{*}\left(G_{n}\left(T X_{0}\right)\right)
$$

has as basis the 1 -forms $\theta^{\alpha}$, and these forms generate the differential ideal giving the canonical exterior differential system $\mathscr{L}_{1}$ on $G_{n}\left(T X_{0}\right)$. The property summarized by the diagram (1) is apparent.

Next, we recall that an integral element of $\mathscr{J}_{0}$ is given by a linear subspace $E \subset T_{p} X_{0}$ such that

$$
\left.\theta\right|_{E}=0, \quad \theta \in \mathscr{J}_{0} .
$$

The set of all $n$-dimensional integral elements will be denoted by $G_{n}\left(\mathscr{J}_{0}\right) \subset$ $G_{n}\left(T X_{0}\right)$. We assume that $G_{n}\left(\mathscr{J}_{0}\right)$ is a smooth manifold with defining equations (2), and then the first prolongation $\left(X_{1}, \mathscr{F}_{1}\right)$ of $\left(X_{0}, \mathscr{I}_{0}\right)$ is defined to be the restriction to $G_{n}\left(\mathscr{F}_{0}\right)$ of the canonical Pfaffian system $\mathscr{L}_{1}$ on $G_{n}\left(T X_{0}\right)$. By what has been said above, the integral manifolds of $\mathscr{I}_{1}$ and $\mathscr{F}_{0}$ are in one-to-one correspondence. In local coordinates, the equations (2) are

$$
F^{\lambda}\left(x^{i}, u^{\alpha}, p_{i}^{\alpha}\right)=0
$$

where $F^{\lambda}$ is a function of the special form

$$
\sum f_{\alpha_{1} \ldots \alpha_{k}}^{i_{1} \ldots i_{k}}(x, u) \operatorname{det}\left\|p_{i_{v}}^{\alpha_{v}}\right\|,
$$

expressing the important fact that the defining equations of $G_{n}\left(\mathscr{F}_{0}\right)$ are the exterior equations (2). Thus the case of a classical PDE system, of the PDE system imposed on sections of a fibered manifold, and of the first prolongation of an exterior differential system all "look the same" in appropriate local coordinates, except that in the last case the defining equations are of a special form. In each of the first two cases there is a canonically associated exterior differential system, and all three of the Pfaffian differential systems are generated by 1 -forms having the same expression in local coordinates. Although we shall use the PDE system imposed on sections of a fibered manifold for illustrative purposes, it is the last of the above contexts in which we shall be working.

Returning to our general discussion, each higher prolongation $\left(X_{k}, \mathscr{J}_{k}\right)$ is defined inductively to be the first prolongation of $\left(X_{k-1}, \mathscr{I}_{k-1}\right)$. This leads to 
the prolongation tower

(3)

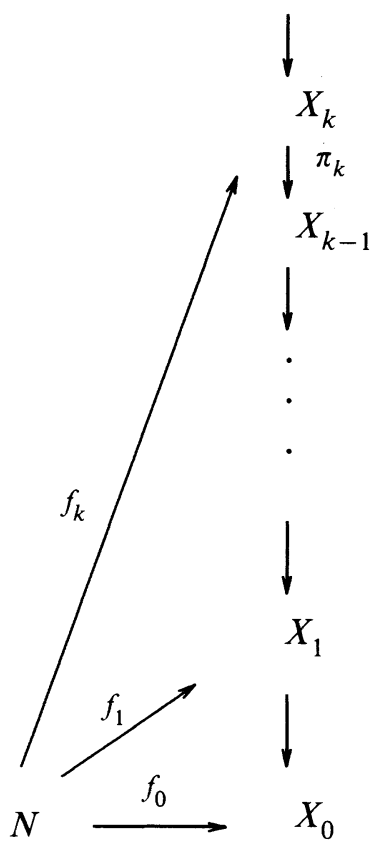

with the property that the integral manifolds $f_{k}: N \rightarrow X_{k}$ of $\mathscr{I}_{k}$ are in one-toone correspondence with the integral manifolds $f_{0}: N \rightarrow X_{0}$ of $\mathscr{I}_{0}$. Intuitively, for $q \in N$ the point $f_{k}(q)$ in $X_{k}$ is the $k$-jet of $f_{0}$ at the point $q$.

Now we shall make the following

Regularity Assumption. The $X_{k}$ are smooth manifolds and $X_{k} \rightarrow X_{k-1}$ is a surjective submersion.

This assumption is satisfied if, e.g., we restrict attention to the projection to $X_{0}$ of a neighborhood of a regular integral element $E_{0}$ (cf. Chapter III of [2]) and take $X_{1}$ to be this neighborhood. That $E_{0}$ should have this property is in practice usually easy to verify using Cartan's test.

With this assumption there are inclusions

$$
\begin{array}{ccc}
\pi_{k}^{*}: \Omega^{*}\left(X_{k-1}\right) & \longrightarrow & \Omega^{*}\left(X_{k}\right) \\
\cup & \cup \\
\pi_{k}^{*}: \mathscr{I}_{k-1} & \longrightarrow & \mathscr{I}_{k}
\end{array}
$$

and, omitting reference to the $\pi_{k}^{*}$, we define

$$
\begin{aligned}
X & =\longleftarrow \lim _{k} X_{k}, \\
\Omega^{*}(X) & =\bigcup_{k \geqq 0} \Omega^{*}\left(X_{k}\right), \\
\mathcal{I} & =\bigcup_{k \geqq 0} \mathscr{I}_{k} .
\end{aligned}
$$


A point of $X$ is an infinite jet of an integral manifold of $\mathscr{I}_{0}$, and by definition a differential form on $X$ is a form on some $X_{k_{0}}$ (or, equivalently, forms $\varphi_{k}$ on $X_{k}$ for $k \geqq k_{0}$ with the coherence property that $\pi_{k}^{*} \varphi_{k-1}=\varphi_{k}$ for $\left.k \geqq k_{0}+1\right)$. The space $X$ is the setting for the "formal differential geometry" of the Russian school and others. The infinite dimensionality of $X$ will not be an issue for us, and we refer to [1] and [11] for general discussions about the rules for doing "calculus" on $X$.

Our basic object of study is given by the following

Definition. Let $\mathscr{J}_{0}$ be an exterior differential system on a manifold $X_{0}$, and construct the prolongation tower (3). Then the characteristic cohomology $\bar{H}^{*}$ is by definition the cohomology of the complex $\left\{\Omega^{*}(X) / \mathscr{I}, d\right\}$. Setting $\bar{\Omega}^{*}=$ $\Omega^{*}(X) / \mathscr{I}$ and $\bar{d}=d \bmod \mathscr{I}$ we thus have

$$
\bar{H}^{*}=H\left\{\bar{\Omega}^{*}, \bar{d}\right\} .
$$

It is useful to examine the prolongation tower construction in local coordinates. We first do this in the unconstrained case-i.e., where $\mathscr{I}_{0}$ is zero-and for this we denote by $G_{n, k}\left(X_{0}\right)$ the space of $k$-jets of $n$-dimensional submanifolds of $X$. Observe that $G_{n, 1}\left(X_{0}\right)=G_{n}\left(T X_{0}\right)$ in our previous notation, that $X_{k} \subset G_{n, k}\left(X_{0}\right)$, and that there is a "universal prolongation tower"

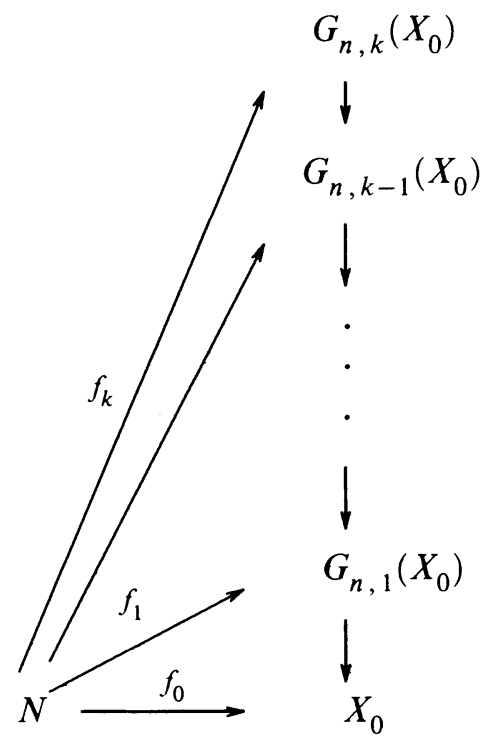

containing (3) as a subtower. Generalizing the above local coordinate system on $G_{n, 1}\left(X_{0}\right)$-which amounts to locally in $G_{n, 1}\left(X_{0}\right)$ representing $n$-dimensional submanifolds of $X_{0}$ as graphs-there are local coordinates

$$
\left(x^{i}, u^{\alpha}, p_{i}^{\alpha}, p_{i j}^{\alpha}, \ldots, p_{I}^{\alpha}\right), \quad|I| \leqq k,
$$

in $G_{n, k}\left(X_{0}\right)$. Here $p_{i j}^{\alpha}=p_{j i}^{\alpha}$, and in general we are using standard multi-index 
notation $I=\left(i_{1}, \ldots, i_{k}\right)$ where $p_{I}^{\alpha}$ is symmetric in the $i_{v}$ (think of $p_{I}^{\alpha}$ as representing $\left.\partial^{k} u^{\alpha} / \partial x^{I}\right)$. The canonical contact system $\mathscr{L}_{k}$ on $G_{n, k}\left(X_{0}\right)$ is the Pfaffian differential system generated locally by the 1 -forms

$$
\begin{aligned}
\theta^{\alpha} & =d u^{\alpha}-p_{i}^{\alpha} d x^{i}, \\
\theta_{i}^{\alpha} & =d p_{i}^{\alpha}-p_{i j}^{\alpha} d x^{j}, \\
& \vdots \\
\theta_{I}^{\alpha} & =d p_{I}^{\alpha}-p_{I i}^{\alpha} d x^{i}, \quad|I|=k-1 .
\end{aligned}
$$

As in the case $k=1$ we can give a coordinate free description of $\mathscr{L}_{k}$, which amounts to saying that the $\theta_{I}^{\alpha},|I| \leqq k-1$, span the annihilator to the span in $T G_{n, k}\left(X_{0}\right)$ of all tangent spaces to $k$-jets of $n$-dimensional submanifolds of $X_{0}$. From

$$
d \theta_{J}^{\alpha}=-d p_{J i}^{\alpha} \wedge d x^{i}=-\theta_{J i}^{\alpha} \wedge d x^{i}, \quad|J| \leqq k-2,
$$

we obtain the following simple but basic fact:

Let $\boldsymbol{\Theta}_{k}$ denote the 1-forms in $\mathscr{L}_{k}$ and $\left\{\boldsymbol{\Theta}_{k}\right\} \subset \Omega^{*}\left(G_{n, k}\left(X_{0}\right)\right)$ the algebraic ideal they generate. Then

$$
d \boldsymbol{\Theta}_{k-1} \subseteq\left\{\boldsymbol{\Theta}_{k}\right\} .
$$

In other words: The universal prolongation tower is constructed by successively adjoining higher derivatives as new variables. This is reflected in the sequence of Pfaffian systems $\mathscr{L}_{1} \subset \mathscr{L}_{2} \subset \cdots$ by the statement that $\mathscr{L}_{k-1}$ satisfies the Frobenius integrability condition (4) relative to $\mathscr{L}_{k}$. In particular, if we pass to jets of infinite order and set

$$
\begin{aligned}
G_{n}\left(X_{0}\right) & =\lim G_{n, k}\left(X_{0}\right), \\
\mathscr{L} & =\bigcup_{k \geqq 0} \mathscr{L}_{k},
\end{aligned}
$$

then $\mathscr{L}$ is formally a Frobenius system on $G_{n}\left(X_{0}\right)$. Since $G_{n}\left(X_{0}\right)$ is not a finite-dimensional manifold, we cannot of course apply the usual Frobenius theorem to conclude that $\mathscr{L}$ defines a true foliation. However, at least informally we may think of the leaves of this "foliation" as being the infinite jets of $n$-dimensional submanifolds of $X_{0}$.

By construction, these considerations restrict to the prolongation tower (3) defined by the original exterior differential system $\mathscr{F}_{0}$ on $X_{0}$. Using the notation

$$
\begin{aligned}
\mathscr{F} & =\text { functions on } X, \\
\Omega^{*} & =\Omega^{*}(X), \\
\Omega_{k}^{*} & =\text { differential graded subalgebra of } \Omega^{*} \text { given by } \Omega^{*}\left(X_{k}\right), \\
\boldsymbol{\Theta}_{k} & =\mathscr{I}_{k} \cap \boldsymbol{\Omega}^{1}=\mathscr{F} \cap \boldsymbol{\Omega}_{k}^{1}, \\
\left\{\boldsymbol{\Theta}_{k}\right\} & =\text { algebraic ideal in } \boldsymbol{\Omega}^{*} \text { generated by } \boldsymbol{\Theta}_{k},
\end{aligned}
$$


the basic observation is then that

$$
d \Theta_{k} \subset\left\{\Theta_{k+1}\right\} \text {. }
$$

Since $\mathscr{I}_{k}$ is generated as a differential ideal by $\Theta_{k}$, we may think of the construction of the prolongation tower (3) as being reflected algebraically by extensions

$$
\begin{array}{cccc}
\mathscr{I}_{k} & \subset & \Omega_{k} \\
\cup & & \cup \\
\mathscr{I}_{k-1} & \subset & \Omega_{k-1}
\end{array}
$$

with the property that $\mathscr{J}_{k-1}$ is closed up in $\Omega_{k}$ relative to the Frobenius integrability condition. We shall formalize this in Section 2.3 below.

1.4 Variation of characteristic cohomology. Let $N$ be a compact manifold and consider integral manifolds

$$
f: N \rightarrow X
$$

of $\mathscr{I}$. We shall identify two integral manifolds when the corresponding immersions differ by a diffeomorphism of $N$, and we denote by $\mathscr{M}$ the set of such equivalence classes. We are interested in how the characteristic cohomology $f^{*}\left(\bar{H}^{*}\right) \subset H_{\mathrm{DR}}^{*}(N)$ varies with $f$, and we observe that this is really a question concerning equivalence classes of integral manifolds. In discussing this question we shall argue formally. Thus, we assume that $\mathscr{M}$ is a "reasonable" space and that there is a diagram

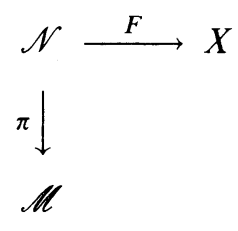

where, if for $t \in \mathscr{M}$ we set

$$
\begin{aligned}
N_{t} & =\pi^{-1}(t), \\
f_{t} & =F \mid N_{t},
\end{aligned}
$$

then $N_{t}$ is diffeomorphic to $N$ and under this diffeomorphism $f_{t}$ represents the equivalence class of the integral manifold given by $t \in \mathscr{M}$.

We denote by $\mathscr{J} \subset \Omega^{*}(\mathscr{N})$ the differential ideal generated by $\Omega^{1}(\mathscr{M})$; the maximal integral manifolds of $\mathscr{J}$ are just the $N_{t}$ 's. By construction we have

$$
F^{*}(\mathscr{I}) \subseteq \mathscr{J} \text {. }
$$

Now for any differential graded algebra $\left\{\Phi^{*}, d\right\}$ and differential ideal $\mathscr{K} \subset$ $\Phi^{*}$ there is a $d$-invariant filtration

$$
F^{p} \mathscr{K}=\text { image }\{\underbrace{\mathscr{H} \wedge \cdots \wedge \mathscr{K}}_{p} \wedge \Phi^{*} \rightarrow \Phi^{*}\} .
$$

The standard spectral sequence construction associated to a filtered complex then gives a spectral sequence $E_{r}^{p, q}(\mathscr{K})$ where

$$
\begin{aligned}
E_{\infty} & \Longrightarrow H^{*}\left(\Phi^{*}\right), \\
E_{1}^{p, q}(\mathscr{K}) & =H^{*}\left(\mathrm{Gr}^{p} \mathscr{K}\right) ;
\end{aligned}
$$


here $\operatorname{Gr}^{p} \mathscr{K}=F^{p} \mathscr{K} / F^{p+1} \mathscr{K}$. This construction is functorial, and thus in our situation there is an induced mapping

$$
F^{*}: E_{r}^{p, q}(\mathscr{J}) \longrightarrow E_{r}^{p, q}(\mathscr{J})
$$

We note that

$$
E_{1}^{0, *}(\mathscr{I})=\bar{H}^{*}
$$

is the characteristic cohomology of the infinitely prolonged differential ideal $\mathscr{I}$. This is the object of our study.

Turning to the fibration $\mathscr{N} \rightarrow \mathscr{M}$, we recall that there is a vector bundle, known informally as the "cohomology bundle", $\mathscr{H}^{q} \rightarrow \mathscr{M}$ whose fiber over $t \in \mathscr{M}$ is $H_{\mathrm{DR}}^{q}\left(N_{t}\right)$ and whose space of global sections is $E_{1}^{0, q}(\mathscr{J})$. Moreover, this bundle has an integrable (i.e., flat) connection

$$
\nabla: \mathscr{H}^{q} \rightarrow \mathscr{H}^{q} \otimes \Omega^{1}(\mathscr{M}),
$$

known as the Gauss-Manin connection, whose horizontal sections are interpreted as geometrically displacing cycles. Now $E_{1}^{1, q}(\mathscr{J}) \cong \mathscr{H}^{q} \otimes \Omega^{1}(\mathscr{M})$ and it is well known (and easy to see) that the differential in the spectral sequence

$$
d_{1}: E_{1}^{0, q} \rightarrow E_{1}^{1, q}
$$

is the Gauss-Manin connection. For $\varphi \in \mathscr{H}^{q}$ this gives us that

$$
\left.\frac{d}{d t}(\varphi)=\frac{\partial}{\partial t}\right\lrcorner d_{1} \varphi
$$

This equation has the following interpretation: Given a point $t_{0} \in \mathscr{M}$ and tangent vector $\xi \in T_{t_{0}} \mathscr{M}$, the left-hand side of (1) is by definition the derivative at $t_{0}$ of $\varphi$ along any curve in $\mathscr{M}$ with tangent vector $\xi$. By the interpretation of the Gauss-Manin connection, this is just $\langle\nabla \varphi, \xi\rangle_{t_{0}}$, which by our above remark is $\xi\lrcorner d_{1} \varphi$.

Putting this together we see that for $\varphi \in \bar{H}^{*}$

$$
\left.\frac{d}{d t}\left(f_{t}^{*} \varphi\right)=\frac{\partial}{\partial t}\right\lrcorner d_{1} \varphi
$$

where the $\partial / \partial t$ on the right-hand side is interpreted as the normal vector along $f_{t}(N)$ corresponding to the tangent vector $\partial / \partial t \in T_{t} \mathscr{M}$.

Thus, the variation of characteristic cohomology is measured by the differential $d_{1}$ in the spectral sequence associated to the differential ideal $\mathscr{I} \subset \Omega^{*}(X)$.

Definition. We set $E_{r}^{p, q}=E_{r}^{p, q}(\mathscr{J})$ and call $E_{r}^{p, q}$ the characteristic spectral sequence associated to the differential ideal $\mathscr{I}$. In particular,

$$
E_{1}^{0, *}=\bar{H}^{*}
$$

is the characteristic cohomology associated to $\mathscr{I}$.

One point of this discussion is that once we agree to study the characteristic cohomology as an interesting object, then the characteristic spectral sequence naturally and inevitably enters. At a deeper level, we will see that our object of interest $\bar{H}^{*}$ is in fact best studied indirectly in terms of the $E_{r}^{p, *}$ for $p>0$. For 
example, the symbol of the exterior differential system will allow us to compute a first approximation to $E_{1}^{p, *}$ for $p>0$.

In summary, we begin by studying the naïve characteristic cohomology $\bar{H}_{0}^{*}$ associated to a differential ideal $\mathscr{I}_{0}$ on a manifold $X$. The necessity of considering derivatives of all orders leads us to replace $X_{0}$ and $\mathscr{I}_{0}$ with the infinitely prolonged exterior differential system $\mathscr{I}_{0}$ on $X$ with its corresponding characteristic cohomology $\bar{H}^{*}$. But then variational considerations lead us to study the entire characteristic spectral sequence $E_{r}^{p, q}$ of which $\bar{H}^{*}=E_{1}^{0, *}$. We shall next isolate the essential algebraic construction which enables us to do this.

\section{FROBENIUS EXTENSIONS}

When one prolongs or differentiates a PDE system up to arbitrary order, what emerges is a filtered object together with differential operators having the property that on the associated graded the operators are algebraic-i.e., they are linear over the functions and depend only on the symbol on highest order terms of the original system. This led Spencer to the introduction of the "Spencer cohomology groups" associated to a symbol, and opened the way for a formal theory that greatly extended and clarified earlier work of Cartan and others. In the present problem of understanding the characteristic cohomology, a similar circumstance will obtain, albeit with one quite new and interesting twist. Underlying this development is a very simple and, we feel, very basic concept in what might be called exterior differential algebra. This concept is that of a Frobenius extension of differential graded algebras relative to a Pfaffian differential ideal. This section will be devoted to a discussion centering around that concept, and ultimately pointing the way to a new and somewhat stranger, although seemingly basic, algebraic construction associated to a symbol.

2.1. Structure equations of the prolongation tower. We shall arrive at the structure equations of the prolongation tower in a number of steps. To explain these we recall that each of the three constructions of Section 1.2

$$
R \subset J^{1}(N, Y) \quad \text { (classical PDE system) }
$$

(iii) $\quad X_{1} \subset G_{n}\left(T X_{0}\right)$

(PDE system associated to a fibered manifold)

(1st prolongation of an exterior differential system)

"looks" the same in local coordinates $\left(x^{i}, u^{\alpha}, p_{i}^{\alpha}\right)$, except that the defining equations of $X_{1}$ have a special form reflecting the fact that they arise from an exterior differential system, which is immaterial for the present discussion. What is different are the symmetry groups associated to the three situations. We shall first give a local coordinate description of the structure equations of the prolongation tower that is invariant under the gauge group associated to construction (ii) above. Our second step will then be to reformulate these equations so as to be invariant under the full symmetry group of generalized contact transformations associated to construction (iii).

We begin by studying some constructions associated to the fibered manifold $Z \rightarrow N$ in the unconstrained case; i.e., when $R=J^{1}(N, Z)$. Denote by $J^{k}(N, Z)$ the manifold of $k$-jets $j^{k}(s)$ of sections $s: N \rightarrow Z$ of the fibered 
manifold, and by $J^{\infty}(N, Z)=\lim ^{k} J^{k}(N, Z)$ the space of jets of infinite order $j^{\infty}(s)$. In coordinates the projection $J^{\infty}(N, Z) \rightarrow N$ is given by

$$
\pi\left(x^{i}, u^{\alpha}, \ldots, p_{I}^{\alpha}, \ldots\right)=\left(x^{i}\right) .
$$

We shall interpret the complete integrability of the infinitely prolonged contact system as giving a flat connection for the fibration $J^{\infty}(N, Z) \rightarrow N$.

For this we recall that functions $G$ on $J^{\infty}(N, Z)$ are given by functions $G\left(x^{i}, u^{\alpha}, \ldots, p_{I}^{\alpha}\right),|I| \leqq k$, on some finite jet manifold $J^{k}(N, Z)$. Letting $\theta_{I}^{\alpha}=d p_{I}^{\alpha}-p_{I i}^{\alpha} d x^{i}$ denote the generators of the contact system on $J^{\infty}(N, Z)$ associated to our choice of local coordinates, we define operators $d_{V}, d_{H}$ by

$$
\begin{aligned}
d_{V} G & =\sum_{|I| \geqq 0} \frac{\partial G}{\partial p_{I}^{\alpha}} \theta_{I}^{\alpha}, \\
d G & =d_{V} G+d_{H} G .
\end{aligned}
$$

Explicitly,

$$
d_{H} G=\sum_{i} D_{i} G d x^{i}
$$

where

$$
D_{i}=\frac{\partial}{\partial x^{i}}+\sum_{|I| \geqq 0} p_{I i}^{\alpha} \frac{\partial}{\partial p_{I}^{\alpha}}
$$

is the operation of "total differentiation with respect to $x^{i}$ "-i.e.,

$$
\frac{\partial}{\partial x^{i}}\left(G\left(j^{\infty}(s)\right)\right)=\left(D_{i} G\right)\left(j^{\infty}(s)\right) \text {. }
$$

The $D_{i}$ are commuting vector fields on $J^{\infty}(N, Z)$ that satisfy

$$
\left\langle\theta_{I}^{\alpha}, D_{i}\right\rangle=0 \text {, }
$$

and thus they span an integrable distribution on $J^{\infty}(N, Z)$ that is horizontal relative to the projection $\pi: J^{\infty}(N, Z) \rightarrow N$. In fact, (1) expresses the fact that $D_{i}$ is the horizontal lift of $\partial / \partial x^{i}$ relative to the integrable connection for $J^{\infty}(N, Z) \rightarrow N$.

Under the decomposition on $J^{\infty}(N, Z)$

$$
d=d_{V}+d_{H}
$$

of the exterior derivative into vertical and horizontal components, we have

$$
\left\{\begin{array}{l}
d_{V}^{2}=d_{H}^{2}=0 \\
d_{V} d_{H}+d_{H} d_{V}=0 .
\end{array}\right.
$$

The decomposition (2) and integrable connection are gauge invariant, as follows, e.g., from the coordinate-free formulation

$$
\left\{\begin{aligned}
d\left(j^{\infty}(s)^{*} G\right) & =j^{\infty}(s)^{*}\left(d_{H} G\right), \\
j^{\infty}(s)^{*}\left(d_{V} G\right) & =0
\end{aligned}\right.
$$

of (1). 
We note that

$$
\left\{\begin{array}{l}
d_{V} \theta_{I}^{\alpha}=0 \\
d_{H} \theta_{I}^{\alpha}=-\theta_{I i}^{\alpha} \wedge d x^{i}
\end{array}\right.
$$

and that trivially $d_{V}\left(d x^{i}\right)=d_{H}\left(d x^{i}\right)=0$. The second of the bracketed equations is equivalent to

$$
\mathscr{L}_{D_{i}}\left(\theta_{I}^{\alpha}\right)=\theta_{I i}^{\alpha}
$$

where $\mathscr{L}_{D}$ is the Lie derivative operator associated to the vector field $D$ on $J^{\infty}(N, Z)$. tions

Suppose now that a constraint manifold $R_{1} \subset J^{1}(N, Z)$ is defined by equa-

$$
F^{\lambda}\left(x^{i}, u^{\alpha}, p_{i}^{\alpha}\right)=0 .
$$

In PDE theory one defines the $k$-th prolongation $R_{k} \subset J^{k}(N, Z)$ of $R$ to be the PDE system defined by the prolonged equations

$$
D_{I} F^{\lambda}=0, \quad|I| \leqq k-1,
$$

where $D_{i_{1} \cdots i_{\ell}}=D_{i_{1}} \cdots D_{i_{\ell}}$. Recall our assumption that each $R_{k}$ is a manifold and that the projections $R_{k} \rightarrow R_{k-1}$ are surjective. We set $R=\lim R_{k}$ and have a fibration diagram:

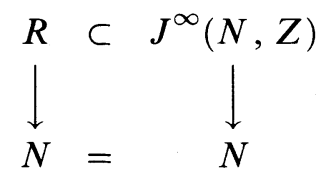

The pertinent observation is that the exterior differential system associated to the PDE system $R_{k}$ is just the $k$-th prolongation, as defined in Section 1.2, of the exterior differential system associated to $R_{1}$. Thus, our terminology is unambiguous and we may say that the infinite prolongation is defined by the equation

$$
D_{I} F^{\lambda}=0 \text {. }
$$

Of course, we may also give the above construction in a coordinate-free manner. For example, $R_{k}$ is simply the set of $k$-jets of formal solutions to the given PDE system, where a $k$-jet of a solution is given by a smooth section of $Z \rightarrow N$ that satisfies the equation up to order $k$ at one point of $N$. We have used the notation $R_{k}$ and $R$ to emphasize that this discussion is invariant under gauge transformation of local coordinates, but is not necessarily invariant under full contact transformations.

The equations defining $R$ are invariant under the horizontal vector fields $D_{i}$ (actually, all that is needed is that the defining ideal of $R \subset J^{\infty}(N, Z)$ be invariant), and thus along $R$ the horizontal spaces are tangent to $R$. We conclude that the fibration $R \rightarrow N$ has a gauge invariant integrable connection, and we shall now denote by $D_{i}, \theta_{I}^{\alpha}, d=d_{V}+d_{H}$ the restrictions to $R$ of the quantities defined above on $J^{\infty}(N, Z)$. 
The vertical forms $\theta_{I}^{\alpha}$ are no longer linearly independent on $X$, but rather satisfy the relations that define $T R \subset T J^{\infty}(N, Z)$. To conveniently express these relations we recall our notation

$$
\begin{aligned}
\Theta_{k} & =\mathscr{I}_{k} \cap \Omega^{1}(R) \\
& =\operatorname{span}\left\{\theta_{I}^{\alpha}:|I| \leqq k-1\right\} .
\end{aligned}
$$

The equations (1) imply that $d_{H}\left(D_{I} F^{\lambda}\right)=0$ along $R$, i.e., in $\left.T\left(J^{\infty}(N, Z)\right)\right|_{R}$. Thus, when restricted to the submanifold $R$ we have

$$
0=d\left(D_{I} F^{\lambda}\right)=d_{V}\left(D_{I} F^{\lambda}\right) .
$$

Taking the ideal $I$ to be empty, this gives

$$
\begin{aligned}
0= & \frac{\partial F^{\lambda}}{\partial p_{i}^{\alpha}} \theta_{i}^{\alpha}+\frac{\partial F^{\lambda}}{\partial u^{\alpha}} \theta^{\alpha} \\
& \Longrightarrow \frac{\partial F^{\lambda}}{\partial p_{i}^{\alpha}} \theta_{i}^{\alpha} \equiv 0 \bmod \Theta_{1} .
\end{aligned}
$$

Taking $I=\{j\}$, we have

$$
\begin{aligned}
0 & =d_{V}\left(D_{j} F^{\lambda}\right) \\
& =\mathscr{L}_{D_{j}}\left(d_{V} F^{\lambda}\right) \\
& \equiv \frac{\partial F^{\lambda}}{\partial p_{i}^{\alpha}} \theta_{i j}^{\alpha} \bmod \Theta_{2}
\end{aligned}
$$

by (3). Repeating this computation inductively, we obtain the defining relation

$$
\frac{\partial F^{\lambda}}{\partial p_{i}^{\alpha}} \theta_{I i}^{\alpha} \equiv 0 \bmod \Theta_{k+1}, \quad|I|=k,
$$

for $T R$ in $T\left(J^{\infty}(N, Z)\right)$. In conclusion:

With the notation

$$
\begin{aligned}
\Theta_{k} & =\mathscr{I}_{k} \cap \Omega^{1}(X) \\
& =\mathscr{I}^{1} \cap \Omega_{k}
\end{aligned}
$$

we have the filtration

$$
\Theta_{1} \subset \Theta_{2} \subset \cdots \subset \Theta=\mathscr{I}^{1}
$$

of the 1-forms in $\mathscr{I}$. The subspaces $\Theta_{k}$ satisfy the structure equations

$$
d \Theta_{k} \equiv 0 \bmod \left\{\Theta_{k+1}\right\}
$$

and

$$
\Theta_{k} / \Theta_{k-1}=\operatorname{span}\left\{\theta_{I}^{\alpha}:|I|=k-1\right\}
$$

where the $\theta_{I}^{\alpha}$ satisfy the symbol relations

$$
\left(\frac{\partial F^{\lambda}}{\partial p_{i}^{\alpha}}\right) \theta_{I i}^{\alpha} \equiv 0 \bmod \Theta_{k}, \quad|I|=k-1 .
$$


We have observed that a gauge transformation preserves the filtration (4) and preserves the complement of $\Theta$ in $\Omega^{1}(R)$ given by the horizontal 1-forms for the flat connection in $R \rightarrow N$. Suppose now that we consider the original PDE system as an exterior differential system $\mathscr{I}_{1}$ on a manifold $X_{1}$. Of course, $R_{1}$ and $X_{1}$ are diffeomorphic as manifolds but the symmetry group of $X_{1}$ may be strictly larger than that of $R_{1}$, hence we use the different notations. As noted above, the manifold $R_{k}$ underlying the $k$-th prolongation of $R_{1}$ as a PDE system is diffeomorphic to the manifold $X_{k}$ underlying the $k$-th prolongation $\mathscr{I}_{k}$ of the exterior differential system $\mathscr{I}_{1}$. We have pointed out that the exterior differential system corresponding to $R_{k}$ is just $\mathscr{F}_{k}$ on $X_{k}$, but again as before the symmetry group of the latter may be larger. Passing to jets of infinite order, an automorphism of the prolongation tower $\left\{R_{k}\right\}$ of $R_{1}$ will induce an automorphism of the prolongation tower $\left\{X_{k}\right\}$, and hence an automorphism of $X$ preserving $\mathscr{F}$. But an automorphism $T$ of $\mathscr{I}$ need not preserve the complement of $\Theta$ in $\Omega^{1}(X)$; i.e., in coordinates we will only have

$$
\begin{aligned}
T^{*} d x^{i} & \equiv A_{j}^{i} d x^{j} \bmod \Theta \\
T^{*} \theta_{I}^{\alpha} & \equiv 0 \bmod \Theta_{k} \quad \text { for } \quad|I| \leqq k-1 .
\end{aligned}
$$

Nevertheless, the relations (4)-(6) are formulated in a manner invariant under such transformations, hence under $\operatorname{Aut}(\mathscr{F})$, and are therefore valid on $X$.

In summary, the relations (4)-(6) are not only gauge invariant, but are invariant under the full symmetry group of the exterior differential system and hence have meaning on $(X, \mathscr{I})$.

2.2. The symbol relations. In the preceding section we arrived at the structure equations

$$
\left\{\begin{array}{l}
\text { (i) } d \Theta_{k} \equiv 0 \bmod \left\{\Theta_{k+1}\right\}, \\
\text { (ii) } \frac{\partial F^{\lambda}}{\partial p_{i}^{\alpha}} \theta_{I i}^{\alpha} \equiv 0 \bmod \Theta_{k+1}, \quad|I|=k
\end{array}\right.
$$

of the prolongation tower. The first equations are formulated in a coordinatefree manner that is invariant under the full symmetry group of the exterior differential system. In this section we will give a similar intrinsic formulation of (ii).

For this we recall the construction of $G_{n, \infty}\left(X_{0}\right)=\lim G_{n, k}\left(X_{0}\right)$, whose points are jets of infinite order of $n$-dimensional submanifolds of $X_{0}$. For all $k$ including $k=\infty$ there are fibrations

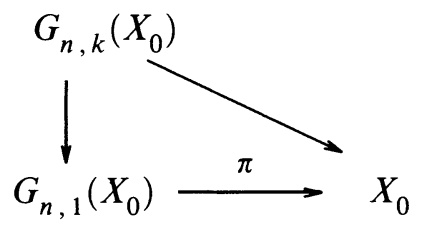


and over $G_{n, 1}\left(X_{0}\right)=G_{n}\left(T X_{0}\right)$ there are defined the tautological vector bundles

$$
\left\{\begin{array}{l}
\underline{E} \subset \pi^{*}\left(T X_{0}\right), \\
\underline{W}=\pi^{*}\left(T X_{0}\right) / \underline{E} .
\end{array}\right.
$$

In terms of the local coordinates used in Section 1.3, $\underline{E}$ has a framing $\partial / \partial x^{1}, \ldots, \partial / \partial x^{n}$ and $\underline{W}$ has a framing $\partial / \partial u^{1}, \ldots, \partial / \partial u^{s}$. We shall continue to denote by $\underline{E}, \underline{W}$ the pullbacks of these bundles to any $G_{n, k}\left(X_{0}\right)$ or to $G_{n, \infty}\left(X_{0}\right)$.

The first basic observation is that over $G_{n, k}\left(X_{0}\right)$ we have exact sequences

$$
0 \rightarrow \underline{W} \otimes S^{k} \underline{E}^{*} \rightarrow T G_{n, k}\left(X_{0}\right) \rightarrow T G_{n, k-1}\left(X_{0}\right) \rightarrow 0
$$

and dually

$$
0 \rightarrow T^{*} G_{n, k-1}\left(X_{0}\right) \rightarrow T^{*} G_{n, k}\left(X_{0}\right) \rightarrow \underline{W^{*}} \otimes S^{k} \underline{E} \rightarrow 0
$$

where $S^{k}$ denotes the $k$-th symmetric product and as usual we are omitting the pullback notation. We shall always lift these sequences up to $G_{n, \infty}\left(X_{0}\right)$, and when we do so and use the local coordinates introduced above, the 1-forms $\theta_{I}^{\alpha},|I|=k$, give a framing for $T^{*} G_{n, k}\left(X_{0}\right) / T^{*} G_{n, k-1}\left(X_{0}\right) \cong \underline{W}^{*} \otimes S^{k} \underline{E}$. For a function $G\left(x^{i}, u^{\alpha}, \ldots, p_{I}^{\alpha}\right),|I| \leqq k$, on $G_{n, k}\left(X_{0}\right)$ the differential $d G$ is an element of $T^{*} G_{n, k}\left(X_{0}\right)$ and the map $T^{*} G_{n, k}\left(X_{0}\right) \rightarrow \underline{W^{*}} \otimes S^{k} \underline{E} \rightarrow 0$ is given by

$$
d G \rightarrow \sum_{|I|=k} \frac{\partial G}{\partial p_{I}^{\alpha}} \theta_{I}^{\alpha} .
$$

The existence of the above sequences is just a notationally complicated way of saying that the highest order derivatives are tensors. The one slight subtlety is that we shall use $\theta_{I}^{\alpha}$ and not $d p_{I}^{\alpha}$ as a coframing of $T^{*} G_{n, k}\left(X_{0}\right) / T^{*} G_{n, k-1}\left(X_{0}\right)$; i.e., we choose our coframing to lie in the contact ideal.

It is when we restrict the second sequence above to the prolongation tower of $\mathscr{F}_{0}$ that the symbol relations appear. More precisely, the pulling back and restriction of differentials associated to the diagram

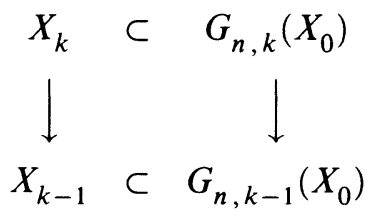

induces on the infinite prolongation $X$ a diagram

$$
\begin{array}{ccccc}
T^{*} G_{n, k}\left(X_{0}\right) / T^{*} G_{n, k-1}\left(X_{0}\right) & \rightarrow & T^{*} X_{k} / T^{*} X_{k-1} & \rightarrow & 0 \\
\mid \approx & & & \mid \approx \\
& \underline{W}^{*} \otimes S^{k} \underline{E} & \rightarrow & \underline{M}_{k} & \rightarrow
\end{array}
$$

where $\underline{B}_{k-1}, \underline{M}_{k}$ are notations. The vector bundle $\underline{M}_{k}$ is spanned by the restrictions of the $\theta_{I}^{\alpha},|I|=k$, to $X$, and the fact that these forms are subject 
to the symbol relations given by equations (ii) in (1) is expressed by saying that $\underline{B}_{k-1} \subset \underline{W}^{*} \otimes S^{k} \underline{E}$ is spanned by the forms

$$
\left(\frac{\partial F^{\lambda}}{\partial p_{i}^{\alpha}}\right) \theta_{J i}^{\alpha}, \quad|J|=k-1
$$

2.3. Frobenius extensions. Most of the calculations in the theory of exterior differential systems have an algebraic, almost algorithmic character; the fact that we are working on a manifold is not the central point, and it may even be distracting. Moreover, many essential points of the theory such as a prolongation and involution are most clearly formulated algebraically, even though they are motivated geometrically. In this and the next section we shall isolate in a purely algebraic formulation what we feel are the essential general aspects of the study of characteristic cohomology of an exterior differential system.

To motivate this discussion, we observe that localizing (i.e., sheafifying) our construction at a point of $X$, we arrive at the data

$$
\left\{\Omega^{*}, \mathscr{J}, \Omega_{k}, d\right\}
$$

where

(i) $\left\{\Omega^{*}, d\right\}$ is a differential graded algebra (DGA),

(ii) $\mathscr{I}$ is a Pfaffian differential ideal,

(iii) $\Omega_{k}^{*}$ is an increasing filtration of $\Omega^{*}$ by subalgebras such that each $\mathscr{J}_{k}=\Omega_{k}^{*} \cap \mathscr{I}$ is a Pfaffian differential ideal.

There are a number of axioms that the data (1) will need to satisfy, of which the essential one is this: Setting $\Theta_{k}=\mathscr{J}_{k}^{1}$ and denoting by $\left\{\Theta_{k+1}\right\}$ the algebraic ideal in $\Omega_{k+1}^{*}$ generated by $\Theta_{k+1}$, we have

Intuitively, the extensions of DGA's

$$
d \Theta_{k} \equiv 0 \bmod \left\{\Theta_{k+1}\right\} \text {. }
$$

$$
\cdots \subset \Omega_{k} \subset \Omega_{k+1} \subset \cdots
$$

are obtained by adjoining 1 -forms such that $\mathscr{I}_{k}$ satisfies the Frobenius integrability condition relative to $\mathscr{I}_{k+1}$.

We formalize this concept with the following

Definition. We consider the data $\left\{\Lambda^{*}, \mathcal{J}, d\right\}$ where $\Lambda^{*}$ is a differential graded algebra and $\mathscr{J} \subset \Lambda^{*}$ is a Pfaffian differential ideal.

(a) The above data is said to be regular in case $\Lambda^{0}$ is a regular local ring and $\Lambda^{*}, \mathscr{I}$ are free modules over $\Lambda^{0}$.

(b) Given an inclusion of regular data

$$
\left\{\Lambda^{*}, \mathscr{J}, d\right\} \subset\left\{\tilde{\Lambda}^{*}, \widetilde{J}, d\right\},
$$

such that $\Lambda^{0}$ and $\bar{\Lambda}^{0}$ have the same residue class field, we say that (2) is a Frobenius extension if

$$
\begin{cases}\text { (i) } & \tilde{\Lambda}^{*} \text { is generated algebraically by } \Lambda^{*} \text { and } \widetilde{\mathcal{J}} \\ \text { (ii) } & \mathscr{J}=\Lambda^{*} \cap \widetilde{\mathcal{J}} \\ \text { (iii) } & d\left(\mathscr{J}^{1}\right) \equiv 0 \bmod \left\{\widetilde{J}^{1}\right\}\end{cases}
$$


(c) The data (1) above where each inclusion $\left\{\Omega_{k}^{*}, \mathscr{I}_{k}, d\right\} \subset\left\{\Omega_{k+1}^{*}, \mathscr{I}_{k+1}, d\right\}$ is a Frobenius extension and where $\Omega^{*}=\bigcup_{k \geqq 0} \Omega_{k}^{*}, \mathscr{I}=\bigcup_{k \geqq 0} \mathscr{I}_{k}$ will be called a Frobenius tower.

As remarked above, intuitively the extension (2) is Frobenius if $\tilde{\Lambda}^{*}$ is obtained from $\Lambda^{*}$ by adjoining 1 -forms (condition (i) above) such that in so doing $\mathscr{J}$ is "closed up" relative to the Frobenius integrability condition ((iii) above), and finally if this extension is non-trivial in the sense that we do not obtain $\widetilde{\mathcal{J}}$ by adding 1 -forms already in $\Lambda^{*}$ (condition (ii)). We may also speak of other properties, such as minimal and universal Frobenius extensions. We shall not enter into such a discussion, but rather we shall explain two conditions on $\{\Lambda, \mathscr{J}, d\}$ that will guarantee that a Frobenius extension exists-this construction will clearly exhibit properties of minimality and universality. These two conditions are motivated by circumstances prevailing in the construction of the prolongation tower of an exterior differential system, which we shall refer to as the geometric situation.

Construction of Frobenius extensions. One may ask when regular data $\left\{\Omega_{1}^{*}, \mathscr{I}_{1}, d\right\}$ admits a Frobenius extension that is in some sense minimal and universal. We are using the notation $\Omega_{1}^{*}, \mathscr{I}_{1}$ rather than $\Lambda^{*}, \mathscr{J}$ to suggest the initial step in the construction of a Frobenius tower. We shall give two conditions on this data which mirror the geometric situation and which guarantee the existence of such a Frobenius extension $\left\{\Omega_{2}^{*}, \mathscr{I}_{2}, d\right\}$. Attempting to continue the process, the first of these conditions will automatically be satisfied for the Frobenius extension $\left\{\Omega_{2}^{*}, \mathscr{I}_{2}, d\right\}$. The second condition, which mirrors the surjectivity of $X_{2} \rightarrow X_{1}$, will have to be assumed at each stage in the construction of the Frobenius tower. However, the first condition will allow us to define what it means for $\left\{\Omega_{1}^{*}, \mathscr{I}_{1}, d\right\}$ to be involutive, and then in the involutive case the second condition will also be satisfied at each stage in the construction of the Frobenius tower.

We set $\mathscr{F}_{1}=\Omega_{1}^{0}$ with residue field $\mathbb{F}$ and $\Theta_{1}=\mathscr{I}_{1}^{1}$. The first condition on the regular data $\left\{\Omega_{1}^{*}, \mathscr{I}_{1}, d\right\}$ is: There exists a subspace $\Omega_{0}^{1} \subset \Omega_{1}^{1}$ such that

$$
d \Theta_{1} \equiv\left(\Omega_{1}^{1} / \Omega_{0}^{1}\right) \wedge \Omega_{0}^{1} \bmod \left\{\Theta_{1}\right\}
$$

In the geometric situation we may take $\Omega_{0}^{1}=\pi^{*} \Omega^{1}\left(X_{0}\right)$ where $\pi: X_{1} \rightarrow X_{0}$ is the projection; the condition (4) follows from the structure equations discussed in Section 2.1 above.

With this assumption there exist free $\mathscr{F}_{1}$-module generators $\theta^{\alpha}$ for $\Theta_{1}$ and $\omega^{i}$ for $\Omega_{0}^{1}$ such that

$$
d \theta^{\alpha} \equiv-\pi_{i}^{\alpha} \wedge \omega^{i} \bmod \left\{\boldsymbol{\Theta}_{1}\right\}
$$

for some elements $\pi_{i}^{\alpha} \in \Omega_{1}^{1}$. These $\pi_{i}^{\alpha}$ are only well defined modulo the $\mathscr{F}_{1}$ span of $\Omega_{0}^{1}$ and $\Theta_{1}$. Moreover, they are not linearly independent but are subject to a minimal set of symbol relations

$$
b_{\alpha}^{\lambda i} \pi_{i}^{\alpha} \equiv 0 \bmod \left\{\Omega_{0}^{1}, \boldsymbol{\Theta}_{1}\right\}
$$


where $b_{\alpha}^{\lambda i} \in \mathscr{F}_{1}$. The non-uniqueness of the $\pi_{i}^{\alpha}$ is expressed by saying that they are well defined modulo $\Theta_{1}$ up to a substitution

$$
\pi_{i}^{\alpha} \rightarrow \pi_{i}^{\alpha}+p_{i j}^{\alpha} \omega^{j}, \quad p_{i j}^{\alpha}=p_{j i}^{\alpha} \in \mathscr{F}_{1} .
$$

The symmetry condition is necessary in order that the equations (5) remain valid. Our second condition is that: There exists a substitution (6) such that the refined symbol relations

$$
b_{\alpha}^{\lambda i} \pi_{i}^{\alpha} \equiv 0 \bmod \Theta_{1}
$$

are satisfied.

As explained in [2], Chapter IV, in the geometric case this condition is equivalent to the assumption that $X_{2} \rightarrow X_{1}$ be surjective. Of course, it can be formulated intrinsically in terms of the data $\left\{\Omega_{1}^{*}, \Omega_{0}^{1}, \mathscr{I}_{1}, d\right\}$ but that would take us too far afield here.

Once we impose the refined symbol relations, the $\pi_{i}^{\alpha}$ are unique up to a substitution (6) where

$$
b_{\alpha}^{\lambda i} p_{i j}^{\alpha}=0, \quad p_{i j}^{\alpha}=p_{j i}^{\alpha} \text {. }
$$

We then define

$$
\mathscr{F}_{2}=\mathscr{F}_{1}\left[p_{i j}^{\alpha}\right]
$$

where the $p_{i j}^{\alpha}$ are indeterminates over $\mathbb{F}$ subject to (7), and we then define $\Omega_{2}^{*}$ to be the DGA generated by $\Omega_{1}^{*}$ and the $p_{i j}^{\alpha}$ and their differentials $d p_{i j}^{\alpha}$, subject of course to the relations obtained by differentiating (7). Finally, we define $\mathscr{I}_{2} \subset \Omega_{2}^{*}$ to be the Pfaffian differential ideal generated as a differential ideal by $\mathscr{I}_{1}$ and the 1 -forms

$$
\theta_{i}^{\alpha}=\pi_{i}^{\alpha}+p_{i j}^{\alpha} \omega^{j} \in \Omega_{2}^{1}
$$

The equation (5) now reads

$$
d \theta^{\alpha} \equiv-\theta_{i}^{\alpha} \wedge \omega^{i} \bmod \left\{\Theta_{1}\right\}
$$

which is the Frobenius extension condition (iii). The other conditions (i) and (ii) are clearly satisfied, and thus $\left\{\Omega_{2}^{*}, \mathscr{J}_{2}, d\right\}$ is a Frobenius extension of $\left\{\Omega_{1}^{*}, \mathscr{I}_{1}, d\right\}$.

We now want to show that condition (4) is inherited by $\left\{\Omega_{2}^{*}, \mathscr{I}_{2}, d\right\}$. Exterior differentiation of the preceding equation gives

$$
0 \equiv-d \theta_{i}^{\alpha} \wedge \omega^{i} \bmod \left\{\boldsymbol{\Theta}_{2}\right\}
$$

Applying the Cartan lemma, we obtain

$$
d \theta_{i}^{\alpha} \equiv-\pi_{i j}^{\alpha} \wedge \omega^{j} \bmod \left\{\Theta_{2}\right\}
$$

where

$$
\pi_{i j}^{\alpha} \equiv \pi_{j i}^{\alpha} \bmod \Theta_{2} .
$$

As before, we may refine this last relation to

$$
\pi_{i j}^{\alpha}=\pi_{j i}^{\alpha} .
$$


Equation (8) is just the first condition (4) for $\left\{\Omega_{2}^{*}, \mathscr{I}_{2}, d\right\}$, where we keep the same subspace $\Omega_{0}^{1}$.

We now investigate the second condition for $\left\{\Omega_{2}^{*}, \mathscr{I}_{2}, d\right\}$. Exterior differentiation of

$$
b_{\alpha}^{\lambda i} \theta_{i}^{\alpha} \equiv 0 \bmod \Theta_{1}
$$

yields, after some fiddling and application of the Cartan lemma, the symbol relations

$$
b_{\alpha}^{\lambda i} \pi_{i j}^{\alpha} \equiv 0 \bmod \Omega_{0}^{1}, \Theta_{2} .
$$

The $\pi_{i j}^{\alpha}$ satisfying (8) and the symmetry condition are uniquely defined up to a substitution

$$
\pi_{i j}^{\alpha} \rightarrow \pi_{i j}^{\alpha}+p_{i j k}^{\alpha} \omega^{k}
$$

where $p_{i j k}^{\alpha} \in \mathscr{F}_{2}$ and is symmetric in $i, j, k$. We must then assume that we may determine $p_{i j k}^{\alpha}$ so as to have the refined symbol relations

$$
b_{\alpha}^{\lambda i} \pi_{i j}^{\alpha} \equiv 0 \bmod \Theta_{2}
$$

In the geometric case this assumption is equivalent to $X_{3} \rightarrow X_{2}$ being surjective. Once it is satisfied, the $\pi_{i j}^{\alpha}$ satisfying (8) and the refined symbol relations are uniquely determined up to a substitution (9) where

$$
b_{\alpha}^{\lambda i} p_{i j k}^{\alpha}=0 \text {. }
$$

We set $\mathscr{F}_{3}=\mathscr{F}_{2}\left[p_{i j k}^{\alpha}\right]$ where the $p_{i j k}^{\alpha}$ are indeterminates over $\mathbb{F}$ subject to these linear equations plus the symmetry in $i, j, k$, and proceed as before to define $\left\{\Omega_{3}^{*}, \mathscr{I}_{3}, d\right\}$ where $\mathscr{I}_{3}$ is the Pfaffian differential ideal generated by

$$
\theta_{i j}^{\alpha}=\pi_{i j}^{\alpha}+p_{i j k}^{\alpha} \omega^{k}
$$

We may continue the argument, constructing a Frobenius tower under the assumptions that the initial data satisfies the condition (4) and that, at each stage, we may refine the symbol relations

$$
b_{\alpha}^{\lambda i} \theta_{I i}^{\alpha} \equiv 0 \bmod \Omega_{0}^{1}, \Theta_{k}, \quad|I|=k,
$$

to

$$
b_{\alpha}^{\lambda i} \theta_{I i}^{\alpha} \equiv 0 \bmod \Theta_{k}, \quad|I|=k .
$$

In so doing the only technical tool required for the inductive step is the CartanPoincaré lemma on page 321 in [2].

Comparing this discussion with the discussion of the structure equations in Section 2.1 and symbol relations in Section 2.2 we see that (4) is satisfied in the geometric case and that moreover the refined symbol relations are satisfied under the assumption that the projections $X_{k+1} \rightarrow X_{k}$ are all submersive.

It is important to remark that there is a condition on the symbol matrices

$$
B(\xi)=\left\|b_{\alpha}^{\lambda i} \xi_{i}\right\|
$$

that will guarantee that the refined symbol relations are satisfied for all $k \geqq 2$, provided only that they are satisfied for $k=1$. This is the condition that the 
symbol be 2-acyclic as defined in [2], Chapter VIII. In the geometric case, the involutivity of the symbol matrices $\left\|\partial F^{\lambda} / \partial p_{i}^{\alpha} \xi_{i}\right\|$ will guarantee that the maps $X_{k+1} \rightarrow X_{k}$ are submersive for all $k \geqq 2$ provided that this is the case for $k=1$. We will then say that the data $\left\{\Omega_{1}^{*}, \mathscr{J}_{1}, d\right\}$ is involutive. As explained in Chapter VI of [2], at least in theory we may always reduce to the involutive case.

In summary, if we begin with a regular DGA $\left\{\Omega_{1}^{*}, \mathscr{I}_{1}, d\right\}$ which satisfies condition (4) above, for which the refined symbol relations hold and for which the symbol is involutive, then we may construct a Frobenius tower $\left\{\Omega^{*}, \Omega_{k}^{*}, \mathscr{I}, d\right\}$. In the next section we will investigate the behavior of this construction under automorphisms (which need not preserve $\Omega_{0}^{1} \subset \Omega_{1}^{1}$ ), and shall show that the Frobenius tower construction leads to a weight filtration on the associated graded to the filtration of $\Omega^{*}$ defined by the powers of $\mathscr{I}$. This weight filtration will have a remarkable property which in practice allows us to get a first approximation to our object of interest $\bar{H}^{*}=H\left\{\bar{\Omega}^{*}, \bar{d}\right\}$ purely from properties of the symbol.

2.4. The weight filtration. Associated to the data $\left\{\Omega^{*}, \mathscr{I}, d\right\}$ we have defined the filtration

$$
F^{p} \Omega^{*}=\text { image of }\{\underbrace{\mathscr{I} \wedge \ldots \wedge \mathscr{I}}_{p} \wedge \Omega^{*} \rightarrow \Omega^{*}\},
$$

to which there is the associated graded complex

$$
F^{p} \Omega^{*} / F^{p+1} \Omega^{*}=: \bar{\Omega}^{p, *}=\bigoplus_{q} \bar{\Omega}^{p, q} .
$$

Suppose now that we have a Frobenius tower $\left\{\Omega^{*}, \mathscr{I}, \Omega_{k}^{*}, d\right\}$. We will use the increasing filtration of $\Omega^{*}$ given by the $\Omega_{k}^{*}$ to define an intrinsic weight filtration on each of the complexes $\left\{\overline{\boldsymbol{\Omega}}^{p, *}, \bar{d}\right\}$, one of which has the remarkable property that on its associated graded the induced differential is linear over the functions, a property traceable to the Frobenius extension condition.

Definition/Proposition. We consider all functions

$$
w: \Omega^{*} \rightarrow \mathbb{Z}
$$

which satisfy

(i) $w(f \varphi)=w(\varphi)$ for $f \in \mathscr{F}, f \neq 0$,

(ii) $w(\varphi) \leqq k$ for $\varphi \in \Theta_{k+1}$, and if equality holds, then $\varphi \notin \Theta_{k}$,

(iii) $w(\varphi \wedge \eta) \leqq w(\varphi)+w(\eta)$, and

(iv) $w(\varphi+\eta) \leqq \max (w(\varphi), w(\eta))$.

We set

$$
\text { wt } \varphi=\max _{w} w(\varphi)
$$

where the maximum is over all functions $w$ satisfying (i)-(iv) above. Then, for $p>0$ "wt" is well defined on the associated graded complexes $\overline{\boldsymbol{\Omega}}^{p, *}$, and if we 
set

$$
\begin{gathered}
\bar{F}_{k} \bar{\Omega}^{p, *}=\left\{\varphi \in \bar{\Omega}^{p, *}: \operatorname{wt}(\varphi) \leqq k\right\}, \\
\bar{\Omega}_{k}^{p, *}=\bar{F}_{k} \bar{\Omega}^{p, *} / \bar{F}_{k-1} \bar{\Omega}^{p, *}=\bigoplus_{q} \bar{\Omega}_{k}^{p, q}
\end{gathered}
$$

we obtain a weight filtration $\left\{\bar{F}_{k}\right\}$ that is stable under $\bar{d}$ and has the property that the induced differential:

$$
\delta: \bar{\Omega}_{k}^{p, q} \rightarrow \bar{\Omega}_{k}^{p, q+1} \text { is } \mathscr{F} \text {-linear } \quad(p>0) .
$$

We shall give the actual construction of the weight filtration under the assumption that $\left\{\Omega_{1}^{*}, \mathscr{I}_{1}, d\right\}$ satisfies (4) in the preceding section and that the refined symbol relations hold at each step. As noted above, these assumptions are both satisfied in the involutive geometric case. Before giving the construction we remark that the crucial property is (1). By means of this property the groups $E_{1}^{p, *}$ (for $p>0$ ) will themselves be the abutment of a spectral sequence, the one coming from the weight filtration whose $E_{1}$-term $\bar{E}_{1}^{k, *}$ will be calculated purely algebraically. It is here that the symbol and Spencer cohomology of the exterior differential system enter the picture, in a more or less standard setting for $p=1$ but in a new and algebraically rather subtle way for $p \geqq 2$.

Construction of the weight filtration. We will follow the notations in the preceding section, especially those used in the construction of Frobenius extensions. Accordingly, we may find a spanning set of forms

$$
\omega^{i}, \theta_{I}^{\alpha}
$$

for $\Omega^{1}$ with the properties that

(a) the $\omega^{i} \in \Omega^{1} \backslash \Theta$,

(b) $\theta_{I}^{\alpha} \in \Theta_{k+1}$ for $|I| \leqq k$,

(c) $b_{\alpha}^{\lambda i} \theta_{I i}^{\alpha} \equiv 0 \bmod \Theta_{k}$ for $|I|=k$.

The relations (iii) are a defining set of relations among the forms $\omega^{i}, \theta_{K}^{\alpha}$.

Now properties (a)-(c) imply that

$$
\left\{\begin{array}{l}
\text { wt } \omega^{i}=-1, \\
\text { wt } \theta_{I}^{\alpha}=|I| \quad\left(\text { provided that } \theta_{I}^{\alpha} \not \equiv 0 \bmod \Theta_{|I|}\right) .
\end{array}\right.
$$

These properties, together with (iii), give

$$
\operatorname{wt}\left(\theta_{I_{1}}^{\alpha_{1}} \wedge \cdots \wedge \theta_{I_{p}}^{\alpha_{p}} \wedge \omega^{j_{1}} \wedge \cdots \wedge \omega^{j_{q}}\right)=\sum_{\nu}\left|I_{\nu}\right|-q
$$

provided of course that the expression on the left is not zero. Finally, (iv) allows us to define wt $\varphi$ for a general form (repeated indices are summed)

$$
\varphi=f_{\alpha_{1} \ldots \alpha_{p} J}^{I_{1} \ldots I_{p}} \theta_{I_{1}}^{\alpha_{1}} \wedge \cdots \wedge \theta_{I_{p}}^{\alpha_{p}} \wedge \omega^{J} .
$$

We must check how "wt" behaves when we make an admissible change of a spanning set of forms (2), and then we must check how "wt" interacts with 
exterior differentiation. For the former, the $\omega^{i}$ are determined up to a transformation

$$
\omega^{i} \rightarrow A_{j}^{i} \omega^{j}+(\text { terms in } \Theta), \operatorname{det}\left\|A_{j}^{i}\right\| \neq 0,
$$

and recalling that $\theta_{I}^{\alpha} \in \Theta_{|I|+1}$ the $\theta_{I}^{\alpha}$ are determined up to a transformation

$$
\theta_{I}^{\alpha} \rightarrow \sum_{|J|=|I|} A_{I \beta}^{\alpha J} \theta_{J}^{\beta}+\left(\text { terms in } \Theta_{|I|}\right), \operatorname{det}\left\|A_{I \beta}^{\alpha I}\right\| \neq 0 .
$$

It follows that (3) is well defined provided that we consider $\theta_{I_{1}}^{\alpha_{1}} \wedge \cdots \wedge \theta_{I_{p}}^{\alpha_{p}} \wedge$ $\omega^{j_{1}} \wedge \cdots \wedge \omega^{j_{q}}$ as an element in $F^{p} \Omega^{*} / F^{p+1} \Omega^{*}$. Put another way, if we assume that the matrices $\left\|A_{j}^{i}\right\|$ and $\left\|A_{I \beta}^{\alpha J}\right\|$ are each the identity, then under the above transformation we have

$$
\begin{gathered}
\theta_{I_{1}}^{\alpha_{1}} \wedge \wedge \wedge \theta_{I_{p}}^{\alpha_{p}} \wedge \omega^{J} \rightarrow \theta_{I_{1}}^{\alpha_{1}} \wedge \cdots \wedge \theta_{I_{p}}^{\alpha_{p}} \wedge \omega^{J}+(\text { terms in } \underbrace{\mathcal{I} \wedge \cdots \wedge \mathcal{I}}_{p+1} \wedge \Omega^{*}) \\
+\left(\text { terms spanned by forms } \theta_{\tilde{I}_{1}}^{\tilde{\alpha}_{1}} \wedge \wedge \wedge \theta_{\tilde{I}_{p}}^{\tilde{\alpha}_{p}} \wedge \omega^{\tilde{J}}\right. \text { where all } \\
\left.\quad\left|\tilde{I}_{\nu}\right| \leqq\left|I_{\nu}\right|,|J|=|\tilde{J}| \text { and some }\left|\tilde{I}_{\nu}\right|<\left|I_{\nu}\right|\right) .
\end{gathered}
$$

The conclusion is that "wt" is well defined on $\bar{\Omega}^{p, *}=F^{p} \Omega^{*} / F^{p+1} \Omega^{*}$.

We note that in $\bar{\Omega}_{k}^{1, *}$ we have the defining relations

$$
b_{\alpha}^{\lambda i} \theta_{I i}^{\alpha}=0,|I|=k-1 .
$$

More generally, in $\bar{\Omega}_{k}^{p, *}$ the relations among the spanning set of forms

$$
\theta_{I_{1}}^{\alpha_{1}} \wedge \cdots \wedge \theta_{I_{p}}^{\alpha_{p}} \wedge \omega^{J}, \quad \sum_{\nu}\left|I_{\nu}\right|-|J|=k,
$$

are linearly generated by the relations (4).

Finally, it remains to investigate how $\bar{d}=d \bmod \mathscr{I}$ interacts with the weight filtration on $\bar{\Omega}^{p, *}$. For this we recall the structure equations for the spanning set of forms (1)

$$
\left\{\begin{array}{l}
d \omega^{i} \equiv \frac{1}{2} c_{j k}^{i} \omega^{j} \wedge \omega^{i} \bmod \{\boldsymbol{\Theta}\}, \\
d \theta_{I}^{\alpha} \equiv-\theta_{I i}^{\alpha} \wedge \omega^{i} \bmod \left\{\boldsymbol{\Theta}_{k}\right\}, k=|I|+1 .
\end{array}\right.
$$

From these it follows that

$$
\left\{\begin{array}{l}
\bar{d} \omega^{i} \equiv 0 \text { modulo }(\mathscr{I}+\text { terms of lower weight }), \\
\bar{d} \theta_{I}^{\alpha} \equiv-\theta_{I i}^{\alpha} \wedge \omega^{i} \text { modulo (terms of lower weight) }
\end{array}\right.
$$

Moreover, for a function $f$

$$
\begin{aligned}
d f= & \sum f_{i} \omega^{i}+(\text { terms in } \Theta) \\
& \Longrightarrow \quad \bar{d} f=\sum f_{i} \omega^{i} \text { has weight }-1
\end{aligned}
$$


Combining this with (5) we see that in the associated graded spaces to the weight filtration $\bar{\Omega}_{k}^{p, *}$,

$$
\begin{aligned}
& \delta\left(f_{\alpha_{1} \ldots \alpha_{p} J}^{I_{1} \ldots I_{p}} \theta_{I_{1}}^{\alpha_{1}} \wedge \cdots \wedge \theta_{I_{p}}^{\alpha_{p}} \wedge \omega^{J}\right) \\
& \quad=(-1)^{p} \sum_{\nu, i} f_{\alpha_{1} \ldots \alpha_{p} J}^{I_{1} \ldots I_{p}} \theta_{I_{1}}^{\alpha_{1}} \wedge \cdots \wedge \theta_{I_{\nu} i}^{\alpha_{\nu}} \wedge \cdots \wedge \theta_{I_{p}}^{\alpha_{p}} \wedge \omega^{i} \wedge \omega^{J} .
\end{aligned}
$$

This establishes the property (1), and moreover (6) gives us a formula for $\delta$ on $\bar{\Omega}_{k}^{p, *}$ that will be used below.

We note in closing the trivial but important point that there is no alternation of signs with the index $\nu$ on the right-hand side of (6). If we let $S=\mathbb{F}\left[e_{1}, \ldots, e_{n}\right]$, define an $S$-module action by

$$
e_{i} \cdot \theta_{I}^{\alpha}=\theta_{I i}^{\alpha} \otimes \omega^{i}
$$

and extend this to products $\theta_{I_{1}}^{\alpha_{1}} \wedge \cdots \wedge \theta_{I_{p}}^{\alpha_{p}} \otimes \omega^{J}$ to act by the Leibnitz rule (with all plus signs) on $\theta_{I_{1}}^{\alpha_{1}} \wedge \cdots \wedge \theta_{I_{p}}^{\alpha_{p}}$, then the right-hand side of (6) is essentially a Koszul boundary operator for this module structure. We shall explore this in detail in the following sections.

2.5. A variant of Spencer cohomology. We continue with our consideration of a Frobenius tower $\left\{\Omega^{*}, \mathscr{I}, \Omega_{k}^{*}, d\right\}$ of Pfaffian differential ideals. We shall assume that this tower arises by an inductive construction, starting with $\left\{\Omega_{1}^{*}, \mathscr{J}_{1}, d\right\}$, where at each step the two conditions consisting of (3) and the refined symbol relations discussed in Section 2.3 are satisfied. As explained in the preceding section, we may construct the weight filtration on the associated graded complexes $\bar{\Omega}^{p, *}$ for $p>0$, and we may then consider the induced differential

$$
\delta: \bar{\Omega}_{k}^{p, q} \rightarrow \bar{\Omega}_{k}^{p, q+1}
$$

on the associated graded complexes to the weight filtration. From equations (1) and (6) in the preceding section we see that $\delta$ treats the functions $\mathscr{F}=\Omega^{0}$ as constants and the forms in $\Omega_{0}^{1}$ as being closed. Thus, the cohomology of the complex $\left\{\bar{\Omega}_{k}^{p, *}, \delta\right\}$ is for $p>0$ "purely algebraic", and as we shall now see it is a variant of Spencer cohomology. Actually, it will turn out to be a variant in two ways. The first essentially trivial one is that a Poincaré-type duality will intervene. More significantly, for $p=1$ we will find the usual Spencer or Koszul cohomology associated to a module $M$, whereas for $p \geqq 2$ we will obtain Spencer cohomology associated to $\Lambda_{\Delta}^{p} M$ where " $\Lambda_{\Delta}^{p}$ " is an apparently new and to us quite interesting multilinear algebra construction.

Review of Spencer-Koszul cohomology. Let $E$ be a vector space over a field $\mathbb{F}$ of characteristic zero, and set

$$
\begin{aligned}
& S^{k} E=\operatorname{Sym}^{k} E=k \text {-th symmetric product of } E, \\
& S=S E=\bigoplus_{k \geqq 0} S^{k} E=\text { polynomial algebra on } E .
\end{aligned}
$$


Let $N$ be a graded (but not necessarily finitely generated) graded $S$-module and recall the standard definition of Koszul homology: Setting

$$
C_{p}(N)=N \otimes_{\mathbb{F}} \Lambda^{p} E
$$

with action by $S$ given by $f(n \otimes \omega)=f n \otimes \omega$ where $f \in S, n \in N$ and $\omega \in \Lambda^{p} E$, one defines the $S$-linear mapping

$$
\partial: C_{p}(N) \rightarrow C_{p-1}(N)
$$

by the usual formula

$$
\partial\left(n \otimes e_{1} \wedge \cdots \wedge e_{p}\right)=\sum_{i}(-1)^{i} e_{i} \cdot n \otimes e_{1} \wedge \cdots \wedge \widehat{e}_{i} \wedge \cdots \wedge e_{p}
$$

where $n \in N$ and $e_{i} \in E$. Then $\partial^{2}=0$ and the resulting Koszul homology will be denoted by $H_{p}(N)$. Clearly $H_{*}(N)=\bigoplus H_{p}(N)$ is again a graded $S$-module.

Dually we set

$$
C^{q}(N)=N \otimes \Lambda^{q} E^{*}
$$

and define

$$
\delta: C^{q}(N) \rightarrow C^{q+1}(N)
$$

by

$$
\delta \omega\left(e_{1}, \ldots, e_{q+1}\right)=\sum_{i}(-1)^{i} e_{i} \cdot \omega\left(e_{1}, \ldots, \widehat{e}_{i}, \ldots, e_{q+1}\right)
$$

where $\omega \in N \otimes \Lambda^{q} E^{*} \cong N \otimes\left(\Lambda^{q} E\right)^{*}$ and the $e_{i} \in E$. Alternatively, we let Id $\in E \otimes E^{*} \cong \operatorname{Hom}(E, E)$ be the identity and define

$$
\text { Id: } N \otimes \Lambda^{q} E^{*} \rightarrow N \otimes \Lambda^{q+1} E^{*}
$$

by

$$
\operatorname{Id}\left(n \otimes e_{i_{1}}^{*} \wedge \cdots \wedge e_{i_{1}}^{*}\right)=\sum e_{i} n \otimes e_{i}^{*} \wedge e_{i_{1}}^{*} \wedge \cdots \wedge e_{i_{1}}^{*}
$$

where $n \in N$ and $e_{i} \in E$ is a basis with dual basis $e_{i}^{*} \in E^{*}$-thus Id = $\sum_{i} e_{i} \otimes e_{i}^{*}$. Then it is easy to verify that

$$
\delta \omega=-\operatorname{Id} \omega
$$

where $\omega \in C^{q}(N)$. The reason for the minus sign will appear below.

To relate these two constructions, we suppose that $\operatorname{dim} E=n$ and choose a non-zero volume from $\Omega \in \Lambda^{n} E$. Contraction with $\Omega$ gives an isomorphism

$$
i(\Omega) \cdot \Lambda^{q} E^{*} \rightarrow \Lambda^{n-q} E
$$

and a commutative diagram

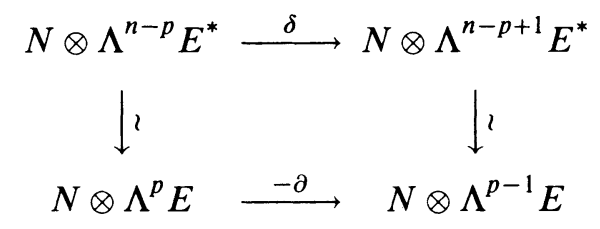

leading to an isomorphism

$$
i(\Omega): H^{n-p}(N) \stackrel{\sim}{\rightarrow} H_{p}(N) .
$$


A different choice of $\Omega$ alters this isomorphism by a non-zero element in $\mathbb{F}$.

We now want to relate this construction to the formula (6) from the preceding section when $p=1$. For this we use the notation

$$
\mathscr{U}_{\bullet}=\mathscr{U} \otimes_{\mathbb{F}} S
$$

for the free $S$-module corresponding to a vector space $\mathscr{U}$. Below we shall take $\mathscr{U}, E$ to be the fibers $W^{*}, E$ of the vector bundles $\underline{W}^{*}, \underline{E}$ introduced in Section 2.2 above. For the moment we assume given vector spaces $\mathscr{U}_{1}, \mathscr{U}_{0}=\mathscr{U}$ and a linear mapping

$$
b: \mathscr{U}_{1} \rightarrow \mathscr{U}_{0} \otimes E,
$$

which we shall refer to as a symbol mapping. In practice, the vector spaces $\mathscr{U}_{1}$, $\mathscr{U}_{0}, E$ will have bases $u^{\lambda}, u^{\alpha}, e_{i}$ such that

$$
b\left(u^{\lambda}\right)=b_{\alpha}^{\lambda i} u^{\alpha} \otimes e_{i}
$$

where the $b_{\alpha}^{\lambda i}$ are the quantities introduced in the preceding section. Associated to (2) is an $S$-linear mapping of free modules

$$
\mathscr{U}_{1} \bullet \stackrel{b}{\longrightarrow} \mathscr{U}_{0} \text { • }
$$

where

$$
b\left(u^{\lambda} e_{I}\right)=b_{\alpha}^{\lambda i} u^{\alpha} e_{i} e_{I}
$$

(we shall omit the $\otimes$ symbol), and we denote by $M$ the cokernel so that we have

$$
\mathscr{U}_{1} \stackrel{b}{\longrightarrow} \mathscr{U}_{0} \bullet \rightarrow M \rightarrow 0 .
$$

The $\mathbb{F}$-basis $u^{\alpha} e_{I}$ of $\mathscr{U}_{0}$. projects to an $\mathbb{F}$-spanning set of vectors $\left[u^{\alpha} e_{I}\right] \in M$ subject to the $\mathbb{F}$-spanning set of relations

$$
b_{\alpha}^{\lambda i}\left[u^{\alpha} e_{i} e_{I}\right]=0 .
$$

$M$ is usually called the graded $S$-module associated to the symbol $b$ and $b\left(\mathscr{U}_{1, .}\right) \subset \mathscr{U}_{0, \text {. }}$ simply the symbol module.

We now denote by $d x^{i} \in E^{*}$ the basis dual to $e_{i}$ and consider the spanning vectors

$$
\left[u^{\alpha} e_{I}\right] \otimes d x^{J} \in C^{q}(M), \quad|J|=q .
$$

Then by our formula (1) above

$$
\delta\left[u^{\alpha} e_{I}\right] \otimes d x^{J}=-\left[u^{\alpha} e_{i} e_{I}\right] \otimes d x^{i} \wedge d x^{J} .
$$

Comparing with the preceding section, we find that the relations (4) are exactly those imposed on the $\theta_{I}^{\alpha}$ considered in the associated graded to the weight filtration, and (5) is exactly the formula (6) in that section for $\delta\left(\theta_{I}^{\alpha} \wedge \omega^{J}\right)$ under the correspondence

$$
\left\{\begin{aligned}
{\left[u^{\alpha} e_{I}\right] } & \longleftrightarrow \theta_{I}^{\alpha}, \\
\omega^{i} & \longleftrightarrow d x^{i} .
\end{aligned}\right.
$$


To be able to reduce the cohomology of $\left\{\bar{\Omega}_{k}^{1, *}, \delta\right\}$ to Spencer cohomology we need to make two further remarks. The first is that for $p>0$ we may consider the cohomology of $\left\{\bar{\Omega}_{k}^{p, *}, \delta\right\}$ as a module over the local ring $\mathscr{F}_{k}=\Omega_{k}^{0}$; this is because $\delta$ is linear over the functions. Denoting by $\mathbf{m}_{\mathbf{k}} \subset \mathscr{F}_{k}$ the maximal ideal, there is a common residue field $\mathbb{F}=\mathscr{F}_{k} / \mathbf{m}_{\mathbf{k}}$ for all $k$. It is the reduction modulo $\mathbf{m}_{\mathbf{k}}$ of the cohomology of $\left\{\overline{\boldsymbol{\Omega}}_{k}^{p, *}, \delta\right\}$ that will be a Spencer-type cohomology, as described in, say, Chapter 8 of [2]. By Nakayama's lemma we will be able to use results about this cohomology to infer information about the cohomology of $\left\{\bar{\Omega}_{k}^{p, *}, \delta\right\}$.

The other remark is that even after we reduce modulo $\mathbf{m}_{\mathbf{k}}$ the $\theta_{I}^{\alpha}$ do not have values in the $k$-th graded piece $M_{k}$ of $M$, but rather have values in $M_{k} \otimes T^{*}$ for some vector space $T$, reflecting the fact that the $\theta_{I}^{\alpha}$ are differential forms. (In the geometric case, $T^{*}=T_{p}^{*} X_{k} / T_{p}^{*} X_{k-1}$.) With these two points understood we may summarize our discussion as follows:

For $p=1$, we consider the reduction modulo $\mathbf{m}_{\mathbf{k}}$ of the complex

$\left\{\bar{\Omega}_{k}^{1, *}, \delta\right\}$. This is then isomorphic to the $k$-th graded piece of $\left\{C^{*}(M) \otimes T^{*}, \delta\right\}$ where $M$ is the graded module associated to the symbol of $\left\{\Omega^{*}, \Omega_{k}^{*}, \mathscr{I}, d\right\}$.

For the sake of explicitness, let us note that we are implicitly using the bigrading

$$
C^{k, q}(M)=\left\{u^{\alpha} e_{I} \otimes d x^{J}:|J|=q,|I|=k+q\right\} .
$$

For $p \geqq 2$ we shall see that a similar conclusion holds with $M$ replaced by $\Lambda_{\Delta}^{p} M$ where " $\Lambda_{\Delta}^{p}$ " is part of the $\Delta$-multilinear algebra formalism to be discussed in the next section.

\section{3. $\Delta$-MULTILINEAR AND COMMUTATIVE ALGEBRA}

The considerations of the preceding section have reduced the computation of the first approximation to the characteristic cohomology to a purely algebraic matter involving a certain type of cohomology constructed from the symbol of the exterior differential system. For $p=1$ we find ordinary Spencer cohomology, but for $p \geqq 2$ a new type of construction appears and it is to this that we now turn, following some preliminary remarks.

3.1. Poincaré polynomials and Spencer cohomology. We keep our previous notation $S=\bigoplus_{k \geqq 0} S^{k} E$ for the symmetric algebra on an $\mathbb{F}$-vector space $E$. We will consider graded $S$-modules $M=\bigoplus_{k \in \mathbb{Z}} M^{k}$ with the properties

(i) $M^{k}=0$ for $k<k_{0}$,

(ii) $\operatorname{dim}_{\mathrm{F}} M^{k}<\infty$ for each $k$.

Such modules will be called quasi-finitely generated-abbreviated QFG. The modules we shall encounter in practice will generally not be finitely generated. Intuitively we may think of a QFG module as being essentially of the form

$$
M=M_{1} \oplus M_{2} \oplus \cdots
$$


where $M_{k}$ is finitely generated in degree $d_{k}$ with $d_{1}<d_{2}<\cdots$. Many of the usual definitions and properties of finitely generated modules carry over to the QFG case.

For example, for a QFG module $M$ we define the Poincaré series of $M$ by the formula

$$
P_{M}(t)=\sum_{i}\left(\operatorname{dim}_{\mathbb{F}} M^{i}\right) t^{i}
$$

it is a Laurent series with a finite tail. If $E$ has dimension $n$, so that upon choosing a basis $e_{1}, \ldots, e_{n}$ we have

$$
S \cong \mathbb{F}\left[e_{1}, \ldots, e_{n}\right],
$$

then

$$
P_{S}(t)=\sum_{i}\left(\begin{array}{c}
n+i-1 \\
i
\end{array}\right) t^{i}=\frac{1}{(1-t)^{n}} .
$$

It follows that for any free quasi-finitely generated $S$-module $F$

$$
(1-t)^{n} P_{F}(t)=\sum_{i} f_{i} t^{i}
$$

where $f_{i}$ is the number of free generators of degree $i$ in any set of free generators of $F$.

If we have an exact sequence of QFG $S$-modules

$$
0 \rightarrow M_{\ell} \stackrel{\varphi_{\ell}}{\longrightarrow} M_{\ell-1} \stackrel{\varphi_{\ell-1}}{\longrightarrow} \cdots \longrightarrow M_{1} \stackrel{\varphi_{1}}{\longrightarrow} M_{0} \rightarrow 0
$$

where $\varphi_{k}$ has degree $\delta_{k}$, then one has the relation

$$
\sum_{k=0}^{\ell}(-1)^{k} t^{\sigma_{k}} P_{M_{k}}(t)=0
$$

where $\sigma_{0}=0$ and $\sigma_{k}=\delta_{1}+\cdots+\delta_{k}$ for $k>0$. More generally, if (1) is a complex with homology modules $H_{i}=\operatorname{ker} \varphi_{i} / \varphi_{i+1}\left(M_{i+1}\right)$, then the $H_{i}$ are quasi-finitely generated $S$-modules and the Euler-Poincaré formula

$$
\sum(-1)^{k} t^{\sigma_{k}} P_{H_{k}}(t)=\sum(-1)^{k} t^{\sigma_{k}} P_{M_{k}}(t)
$$

is valid.

We want to apply this relation to the Spencer cohomology $H^{*}(M)$ of a QFG $S$-module $M$. Recall from the preceding section that Spencer cohomology is constructed from the complex of QFG $S$-modules

$$
\cdots \rightarrow M \otimes_{\mathbb{F}} \Lambda^{q} E^{*} \stackrel{\delta}{\longrightarrow} M \otimes_{\mathbb{F}} \Lambda^{q+1} E^{*} \rightarrow \cdots,
$$

and remark that a number of properties from the usual finitely generated case carry over. For example, if $S^{+}=\bigoplus_{k>0} S^{k}$ denotes the maximal ideal of $S$, then

$$
H^{n}(M) \cong M / S^{+} M
$$

which follows immediately from

$$
\delta\left(\sum_{i}(-1)^{i-1} m_{i} \otimes d x^{1} \wedge \cdots \wedge \widehat{d x^{i}} \wedge \cdots \wedge d x^{n}\right)=\sum_{i}\left(e_{i} m_{i}\right) \otimes d x^{1} \wedge \cdots \wedge d x^{n} .
$$


More generally, the maximal ideal acts trivially on all of $H^{*}(M)$, as follows from the homotopy formula

$$
\mu_{i}=\delta \nu_{i}+\nu_{i} \delta
$$

where $\mu_{i}$ is multiplication by $e_{i}$ and $\left.\nu_{i}(m \otimes \varphi)=m \otimes e_{i}\right\lrcorner \varphi$. In particular, the $H^{q}(M)$ are QFG $\mathbb{F}$-modules-i.e., they are graded $\mathbb{F}$-vector spaces of the form $\bigoplus_{i \geqq i_{0}} V_{i}$ where $V_{i}$ has grade $i$ and $\operatorname{dim}_{\mathbb{F}} V_{i}<\infty$.

If $F$ is a QFG free $S$-module, then it is well known and easy to prove that

$$
H^{q}(F)=0, \quad q<n .
$$

The converse is also true; i.e., if $M$ is QFG and all $H^{q}(M)=0$ for $q<n$, then $M$ is free. This may be seen as follows: Setting $F=S \otimes_{\mathbb{F}} H^{n}(M)$ we have an exact sequence of QFG modules

$$
0 \rightarrow N \rightarrow F \rightarrow M \rightarrow 0
$$

with $H^{n}(F) \stackrel{\sim}{\rightarrow} H^{n}(M)$ by construction. It follows from the assumption (3) and long exact cohomology sequence that $H^{n}(N)=0$, which by our above observation (2) gives that $N=0$ and consequently $F \cong M$.

Generalizing this argument gives, as in the usual case, the Hilbert syzygy theorem for $Q F G$ S-modules: Given a $Q F G$ module $M$ there is a minimal resolution by free $Q F G$ modules

$$
0 \rightarrow F_{\ell} \stackrel{\varphi_{\ell}}{\longrightarrow} F_{\ell-1} \stackrel{\varphi_{\ell-1}}{\longrightarrow} \cdots \longrightarrow F_{1} \stackrel{\varphi_{1}}{\longrightarrow} F_{0} \stackrel{\varphi_{0}}{\longrightarrow} M \rightarrow 0,
$$

where $\varphi_{0}$ has graded degree zero and all the other $\varphi_{i}$ for $i>0$ have positive graded degree, and where

$$
F_{i} \cong S \otimes_{\mathbb{F}} H^{n-i}(M) .
$$

We observe that the resolution length $\ell$ satisfies $\ell \leqq n$.

In particular, for the Poincare polynomials one has the formula

$$
(1-t)^{n} P_{M}(t)=\sum_{q}(-1)^{q} t^{q} P_{H^{n-q}(M)}(t) .
$$

3.2. $\Delta$-tensor and exterior products. Our basic construction is the $\Delta$-tensor product, denoted $M \otimes_{\Delta} N$, of QFG graded $S$-modules $M$ and $N$. The defining properties are

$$
\begin{aligned}
& \left(M \otimes_{\Delta} N\right)^{q}=\bigoplus_{i+j=q} M^{i} \otimes_{\mathbb{F}} N^{j}, \\
& e \cdot(m \otimes n)=e m \otimes n+m \otimes e n, \quad e \in E .
\end{aligned}
$$

It is easy to see that

$$
e^{\prime} \cdot(e \cdot(m \otimes n))=e \cdot\left(e^{\prime} \cdot(m \otimes n)\right)
$$

so that the action of $E$ on $M \otimes_{\mathbb{F}} N$ extends to an action of $S$ on $M \otimes_{\mathbb{F}} N$, thereby defining the $S$-module $M \otimes_{\Delta} N$. The "Leibnitz rule" (ii) is of course what gives interest to this construction. The properties

$$
\begin{aligned}
& M \otimes_{\Delta} N \cong N \otimes_{\Delta} M, \\
&(M \oplus N) \otimes_{\Delta} P \cong\left(M \otimes_{\Delta} P\right) \oplus\left(N \otimes_{\Delta} P\right), \\
&\left(M \otimes_{\Delta} N\right) \otimes_{\Delta} P \cong M \otimes_{\Delta}\left(N \otimes_{\Delta} P\right)
\end{aligned}
$$


hold for the $\Delta$-tensor product of $S$-modules as for ordinary tensor products.

It is not true that the $\Delta$-tensor product of finitely generated modules is again finitely generated. For example, suppose that $S=\mathbb{F}[x]$. We will show that

$$
S \otimes_{\Delta} S \text { has } \quad\left\{x^{k} \otimes 1 ; k \geqq 0\right\} \quad \text { as free generators. }
$$

Proof. Let $M$ be the $S$-submodule generated by the $x^{k} \otimes 1$. Then

$$
x \cdot\left(x^{k} \otimes x^{\ell}\right)-x^{k+1} \otimes x^{\ell}=x^{k} \otimes x^{\ell+1},
$$

so that $x^{k} \otimes x^{\ell+1} \in M$ if $x^{k+1} \otimes x^{\ell}$ and $x^{k} \otimes x^{\ell} \in M$. It follows inductively that $M$ generates $S \otimes_{\Delta} S$. Suppose now that we have a relation

$$
\sum_{k} P_{k}(x) \cdot\left(x^{k} \otimes 1\right)=0
$$

in $S \otimes_{\Delta} S$. Writing $P_{k}(x)=\sum_{\ell} P_{k \ell} x^{\ell}$ we look inductively at the terms of total degree zero, one, two, etc. to obtain

$$
\begin{gathered}
P_{00} 1 \otimes 1=0 \Longrightarrow P_{00}=0, \\
P_{01}(x \otimes 1+1 \otimes x)+P_{10} x \otimes 1=0 \Longrightarrow P_{01}=0, P_{10}=0, \\
P_{02}\left(x^{2} \otimes 1+2 x \otimes x+1 \otimes x^{2}\right)+P_{11}\left(x^{2} \otimes x+x \otimes x\right)+P_{20} x^{2} \otimes 1=0 \\
\Longrightarrow P_{02}=0, P_{11}=0, P_{20}=0 .
\end{gathered}
$$

Continuing in this way we find that all $P_{k}(x)=0$. Q.E.D.

Remark. We may think of (1) as saying that: "By integration by parts in $\int f^{(k)}(x) g^{(\ell)}(x) d x$ we may move all the derivatives to $f$, and no further reductions are possible." This is of course obvious. As we shall see below, what is more interesting is what happens when $f$ and $g$ anti-commute.

A generalization of (1) is given by

Proposition 1. For $S \cong \mathbb{F}\left[e_{1}, \ldots, e_{n}\right]$ the $\Delta$-tensor product $S \otimes_{\Delta} S$ is a free $Q F G$ $S$-module, and in fact is freely generated by the $\mathbb{F}$-subspace $S \otimes_{\Delta} 1 \subset S \otimes_{\Delta} S$.

Proof. We have the relation of Poincare polynomials

$$
P_{S \otimes_{\Delta} S}(t)=P_{S}(t) P_{S}(t)=\frac{1}{(1-t)^{2 n}} .
$$

From

$$
e_{i} m \otimes n \equiv-m \otimes e_{i} n \text { modulo } E \cdot\left(S \otimes_{\Delta} S\right)
$$

it follows that $S \otimes_{\Delta} 1$ generates the entire $S$-module $S \otimes_{\Delta} S$. In particular, the $S$-module mapping

$$
S \otimes_{\mathbb{F}}\left(S \otimes_{\Delta} 1\right) \rightarrow S \otimes_{\Delta} S
$$

given by $m \otimes(n \otimes 1) \rightarrow m \cdot(n \otimes 1)$ is surjective. It also preserves natural gradings, and since by the above Poincare series formula both graded vector spaces have the same dimension in each grade the mapping must be injective as well. Q.E.D. 
Corollary. The $\Delta$-tensor product of any number of free $Q F G S$-modules is also free and $Q F G$.

Consider now the $q$-fold $\Delta$-tensor power of a QFG $S$-module $M$, which is denoted by $\otimes_{\Delta}^{q} M$. The symmetric group on $q$ letters, $S_{q}$, acts on the QFG $S$-module $\otimes_{\Delta}^{q} M$ in the obvious way. Since $\mathbb{F}$ has characteristic zero, there is a direct sum decomposition of $\otimes_{\Delta}^{q} M$ into submodules invariant under the action of $S_{q}$. These submodules are free in case $M$ is free. One of these summands will be of particular interest to us, namely the $q$-th $\Delta$-exterior power $\Lambda_{\Delta}^{q} M \subset \otimes_{\Delta}^{q} M$.

To get some feeling for this construction suppose that $S=\mathbb{F}[x]$. We set

$$
m \wedge n=m \otimes n-n \otimes m \in \Lambda_{\Delta}^{q} S .
$$

Thus by (1) the elements $x^{k} \wedge 1$ generate the free module $\Lambda_{\Delta}^{2} S$. But these are not free generators, since we have the relations

$$
\begin{array}{llrl}
x^{0} \wedge 1 & =0, & k & =0, \\
x^{2} \wedge 1 & =x \cdot(x \wedge 1), & k & =2, \\
x^{4} \wedge 1 & =-x^{3} \cdot(x \wedge 1)+2 x \cdot\left(x^{3} \wedge 1\right), & k & =4 .
\end{array}
$$

We shall see below that this pattern continues, so that we will have the result:

Proposition 2. The elements $x^{2 k+1} \wedge 1, k \geqq 0$, freely generate $\Lambda_{\Delta}^{2} \mathbb{F}[x]$. More generally, for $S=\mathbb{F}\left[e_{1}, \ldots, e_{n}\right]$ the elements

$$
e_{I} \wedge 1, \quad|I|=2 k+1,
$$

where $I=\left(i_{1}, \ldots, i_{2 k+1}\right)$ and $e_{I}=e_{i_{1}} \cdots e_{i_{2 k+1}}$ freely generate $\Lambda_{\Delta}^{2} S$.

Proof. For any $e \in E$ and $m, n \in S$ the defining property

$$
e(m \wedge n)=e m \wedge n+n \wedge e n
$$

implies that $(e m) \wedge n \equiv-m \wedge(e n)$, and so $m \wedge 1 \equiv(-1)^{\operatorname{deg} m} 1 \wedge m$ where $\equiv$ denotes congruence modulo $E \cdot \Lambda_{\Delta}^{2} S$. Thus

$$
e_{I} \wedge 1 \equiv(-1)^{|I|+1} e_{I} \wedge 1
$$

and so $e_{I^{\wedge}} 1 \equiv 0$ for $|I|$ even. It follows from Proposition 1 that the elements $e_{I^{\wedge}} \wedge 1,|I|=2 k+1$, generate $\Lambda_{\Delta}^{2} S$.

We will first show that these are free generators in the case $n=1$ when $S \cong \mathbb{F}[x]$.

Let $V$ be the graded $\mathbb{F}$-vector subspace of $\mathbb{F}[x]$ spanned by the $x^{2 k+1} \wedge 1$ for $k \geqq 0$. There is a surjective $\mathbb{F}[x]$-module mapping

$$
\mathbb{F}[x] \otimes_{\mathbb{F}} V \rightarrow \Lambda_{\Delta}^{2} \mathbb{F}[x]
$$

given by $f \otimes v \rightarrow f \cdot v$. It will suffice to show that the Poincare polynomials of these two modules coincide, and inspection shows that each is given by $\sum_{\ell \geqq 0}\left[\frac{\ell+1}{2}\right] t^{\ell}$ (in each case, we add one new dimension each time the grade goes up by two). 
In general, any relation among the $e_{I} \wedge 1,|I|=2 k+1$, would induce a relation on a line-i.e., when we set $e_{i}=\alpha_{i} x$ where the $\alpha_{i}$ are fixed. If the relation were non-trivial, this would still be the case on a general line in contradiction to the case when $S=\mathbb{F}[x]$ above. Q.E.D.

An alternative argument not reducing to the case $n=1$ arises by computing the Poincaré polynomial of $\Lambda_{\Delta}^{q} M$ in terms of the Poincaré polynomial of $M$, to which we now turn.

Since the $\Delta$-tensor product of two $S$-modules, when regarded as a vector space, is merely the graded tensor product over $\mathbb{F}$ of the underlying graded vector spaces, it easily follows that the following standard formula is valid:

$$
\Lambda_{\Delta}^{q}(M \oplus N) \simeq \bigoplus_{i+j=q}\left(\Lambda_{\Delta}^{i} M \otimes_{\mathbb{F}} \Lambda_{\Delta}^{j} N\right) .
$$

In particular, if the modules $M$ and $N$ are QFG, this implies the following relation on Poincaré series:

$$
P_{\Lambda_{\Delta}^{q}(M \oplus N)}(t)=\sum_{i+j=q} P_{\Lambda_{\Delta}^{i}(M)}(t) P_{\Lambda_{\Delta}^{j}(N)}(t) .
$$

The above observation can be used to develop a formula for the Poincare series of $\Lambda_{\Delta}^{q} M$ in terms of the Poincare series of $M$.

First, a few observations about symmetric polynomials will be useful. Let $y_{1}, y_{2}, \ldots, y_{m}$ be any set of variables and let $s_{i}$ denote the $i$-th elementary symmetric function of the variables $y_{a}$. The power functions of the $y_{a}$ are defined as

$$
p_{i}=\left(y_{1}\right)^{i}+\left(y_{2}\right)^{i}+\cdots+\left(y_{m}\right)^{i} \text {. }
$$

It is well known that the functions $p_{i}$ for $i \leqq m$ freely generate the ring of symmetric functions in the $y_{a}$ and hence that there exist unique polynomial functions $E_{i}\left(p_{1}, p_{2}, \ldots, p_{i}\right)$ so that, independent of $m$, one has

$$
s_{i}=E_{i}\left(p_{1}, p_{2}, \ldots, p_{i}\right) \text {. }
$$

For example, the first four such polynomials are

$$
\begin{aligned}
& E_{1}=p_{1}, \\
& E_{2}=\frac{1}{2}\left(\left(p_{1}\right)^{2}-p_{2}\right), \\
& E_{3}=\frac{1}{6}\left(\left(p_{1}\right)^{3}-3 p_{1} p_{2}+2 p_{3}\right), \\
& E_{4}=\frac{1}{24}\left(\left(p_{1}\right)^{4}-6\left(p_{1}\right)^{2}+8 p_{1} p_{3}+3\left(p_{2}\right)^{2}-6 p_{4}\right) .
\end{aligned}
$$

Now, if $y_{0}$ is another variable, and $s_{i}^{\prime}$ is the $i$-th elementary symmetric function of the variables $y_{0}, y_{1}, y_{2}, \ldots, y_{m}$, then clearly $s_{i}^{\prime}=s_{i}+y_{0} s_{i-1}$. It follows that the polynomial functions $E_{q}$ satisfy the following functional identities for any $y$ :

$E_{q}\left(y+p_{1}, y^{2}+p_{2}, \ldots, y^{q}+p_{q}\right)=E_{q}\left(p_{1}, p_{2}, \ldots, p_{q}\right)+y E_{q-1}\left(p_{1}, p_{2}, \ldots, p_{q-1}\right)$.

Proposition 3. For any $Q F G S$-module $M$, the Poincaré series of its $q$-th $\Delta$ exterior power $\Lambda_{\Delta}^{q} M$ is given by

$$
P_{\Lambda_{\Delta}^{q} M}(t)=E_{q}\left(P_{M}(t), P_{M}\left(t^{2}\right), \ldots, P_{M}\left(t^{q}\right)\right) .
$$


Proof. As has been already remarked, this is really a result about exterior powers of graded vector spaces which are bounded below. Moreover, since the polynomial $E_{q}$ is weighted homogeneous of degree $q$ when the variable $p_{i}$ is assigned the weight $i$, one can clearly reduce to the case where $M^{i}=0$ for all $i<0$ by an elementary shift-of-index argument. Thus, we assume this from now on.

Since $M^{i}=0$ for all $i<0$, it is clear that the $i$-th graded piece of $\Lambda_{\Delta}^{q} M$ is completely determined by the vector spaces $M^{j}$ for $j \leqq i$. It follows that it suffices to prove the above formula in the case that $M$ is a finite-dimensional graded vector space.

Thus, assume that $M$ is finite dimensional. We will now complete the proof by double induction on $q$ and the dimension of $M$. If $q=1$ or the dimension of $M$ is zero, then the formula is clearly true. If $M=N \oplus \mathbb{F} u$ where $u$ is an element of degree $k$, then $P_{M}(t)=P_{N}(t)+t^{k}$. Moreover, by the above formula for exterior powers of sums, we see that $\Lambda_{\Delta}^{q} M \simeq \Lambda_{\Delta}^{q} N \oplus\left(\mathbb{F} u \otimes \Lambda_{\Delta}^{q-1} N\right)$, so

$$
P_{\Lambda_{\Delta}^{q} M}(t)=P_{\Lambda_{\Delta}^{q} N}(t)+t^{k} P_{\Lambda_{\Delta}^{q-1} N}(t)
$$

This formula, coupled with the functional identity on the polynomials $E_{q}$, clearly implies the desired result. Q.E.D.

In particular, consider the case of Proposition 2 above when $S=$ $\mathbb{F}\left[e_{1}, \ldots, e_{n}\right]$. Then

$$
\begin{aligned}
P_{\Lambda_{\Delta}^{2} S}(t) & =E_{2}\left(P_{S}(t), P_{S}\left(t^{2}\right)\right) \\
& =\frac{1}{2}\left(\frac{1}{(1-t)^{2 n}}-\frac{1}{\left(1-t^{2}\right)^{n}}\right) .
\end{aligned}
$$

In particular, we have

$$
\begin{aligned}
(1-t)^{n} P_{\Lambda_{\Delta}^{2} S}(t) & =\frac{1}{2}\left(\frac{1}{(1-t)^{n}}-\frac{1}{(1+t)^{n}}\right) \\
& =\sum_{i \in 2 \mathbb{Z}^{+}+1}\left(\begin{array}{c}
n+i-1 \\
i
\end{array}\right) t^{i}
\end{aligned}
$$

The last term is the Poincare series of any graded $\mathbb{F}$-subspace of $\Lambda_{\Delta}^{2} S$ which freely generates $\Lambda_{\Delta}^{2} S$. It follows again that the monomials $m \wedge 1$ where $m$ has odd degree must be a freely generating set.

Finally, we consider the general free module case where

$$
M \cong W^{*} \otimes_{\mathbb{F}} \mathbb{F}\left[e_{1}, \ldots, e_{n}\right]
$$

for some vector space $W^{*}$ with basis $w^{1}, \ldots, w^{s}$. We then have relations generalizing the one given above,

$$
e_{I} w^{\alpha} \wedge w^{\beta} \equiv(-1)^{|I|+1} e_{I} w^{\beta} \wedge w^{\alpha}
$$


from which we may infer that

$$
\begin{aligned}
& \text { Free generators of } \Lambda_{\Delta}^{2} M \text { are given by } \\
& e_{I} w^{\alpha} \wedge w^{\beta} \text { where } \begin{cases}\alpha \leqq \beta & \text { if }|I| \text { is odd } \\
\alpha<\beta & \text { if }|I| \text { is even; }\end{cases}
\end{aligned}
$$

here it is understood that $e_{I} w^{\alpha} \wedge w^{\beta}=\left(e_{I} w^{\alpha}\right) \wedge w^{\beta}$.

In cohomological terms, if $S^{+}$denotes the maximal ideal of $S$, then

$$
\begin{aligned}
H^{n}\left(\Lambda_{\Delta}^{2} M\right) & \cong \Lambda_{\Delta}^{2} M / S^{+} \Lambda_{\Delta}^{2} M \\
& \cong\left\{\varphi=\varphi_{\alpha \beta}^{I} e_{I} w^{\alpha} \wedge w^{\beta}: \varphi+(-1)^{|I| t} \varphi=0\right\}
\end{aligned}
$$

where ${ }^{t} \varphi=\varphi_{\beta \alpha}^{I} e_{I} w^{\alpha} \wedge w^{\beta}$. A similar result may be proved for the $\Delta$-symmetric product $\operatorname{Sym}_{\Delta}^{2} M$, giving that

$$
H^{n}\left(\operatorname{Sym}_{\Delta}^{2} M\right) \cong\left\{\varphi=\varphi_{\alpha \beta}^{I} e_{I} w^{\alpha} \wedge w^{\beta}: \varphi-(-1)^{|I| t} \varphi=0\right\} .
$$

We now establish contact between the $\Delta$-exterior product construction and the induced differential on the associated graded to the weight filtration. Namely, comparing formula (6) in Section 2.4 with the formula (1) in that section for the coboundary map in Spencer cohomology using the correspondence given by (6) in Section 2.5, we infer that (7) then extends to the general case, as follows:

We consider the reduction modulo $\mathbf{m}_{\mathbf{k}}$ of the complex $\left\{\bar{\Omega}_{k}^{p, *}, \delta\right\}$. This is then isomorphic as a complex to the $k$-th graded piece of $\left\{C^{*}\left(\Lambda_{\Delta}^{p} M\right) \otimes T^{*}, \delta\right\}$, where $M$ is the graded module associated to the symbol of the exterior differential system.

\section{VANISHING OF THE CHARACTERISTIC COHOMOLOGY}

4.1. Characteristic cohomology in the unconstrained case. Recall that the unconstrained case refers to the empty exterior differential system $\mathscr{J}_{0}$ on $X_{0}$; i.e., geometrically we are considering all immersions

$$
f: N \rightarrow X_{0}
$$

of an $n$-manifold $N$ into $X_{0}$. In this case the prolongation tower is described, in the notation of Section 1.3, by

$$
X_{k}=G_{n, k}\left(X_{0}\right)=\left\{\begin{array}{l}
\text { all } k \text {-jets of immersions of } \\
\text { an } n \text {-dimensional manifold into } X_{0}
\end{array}\right\} .
$$

We shall also consider the local case; i.e., we work in the inverse image in $X=G_{n, \infty}\left(X_{0}\right)$ of a particular $n$-plane $E_{0} \in G_{n, 1}\left(X_{0}\right)$. The basic result here is due, in its general form, to Vinogradov [13].

Theorem. In the unconstrained local case

$$
\bar{H}^{q}=0 \text { for } 0<q<n .
$$


Proof. We shall see that the result is a direct consequence of the facts, noted in Sections 3.1 and 3.2 above, that

(i) For $F$ a free QFG $S$-module the Spencer cohomology

$$
H^{q}(F)=0, \quad q<n .
$$

(ii) If $M$ is a free QFG $S$-module, then so are the exterior powers $\Lambda_{\Delta}^{p} M$. Thus by (i)

$$
H^{q}\left(\Lambda_{\Delta}^{p} M\right)=0, \quad q<n .
$$

The first part of the following discussion will apply to the general constrained case. Referring to Section 1.4 , we recall that the filtration of $\Omega^{*}(X)$ by the exterior powers of $\mathscr{I}$ induces a filtration $F^{p}$ on $\Omega^{*}(X)$ such that the resulting spectral sequence has

$$
\begin{aligned}
E_{1}^{0, q} & =\bar{H}^{q}, \\
E_{\infty} & \Rightarrow H_{\mathrm{DR}}^{*}(X) .
\end{aligned}
$$

Since we are working in the local case, $X$ is contractible and thus $E_{\infty}^{p, q}=0$ for $p+q>0$. It will therefore suffice to prove that

$$
E_{1}^{p, q}=0 \text { for } p>0, q<n .
$$

(An important point to take note of here is that we are studying the characteristic cohomology indirectly, i.e., from the knowledge of the $E_{1}^{p, q}$ for $p>0$ and of $E_{\infty}^{p, q}$.)

Set $\bar{\Omega}^{p, *}=F^{p} \Omega^{*} / F^{p+1} \Omega^{*}$ and from Section 2.4 recall the weight filtration defined on $\bar{\Omega}^{p, *}$ for all $p$ but non-trivial only for $p>0$. Fixing $p>0$, there is the weight spectral sequence $\bar{E}_{r}^{k, q}$ which abuts to $E_{1}^{p, q}$ and has

$$
\bar{E}_{1}^{k, *}=H\left(\bar{\Omega}_{k}^{p, *}\right)
$$

where $\bar{\Omega}_{k}^{p, *}$ is the associated graded complex to the weight filtration. Now the main point is that, from (1) in Section $2.4, H\left(\bar{\Omega}_{k}^{p, *}\right)$ is given by sections of a vector bundle over $X$, and from Section 2.5 we see that

The fiber at $\xi \in X$ of $H\left(\bar{\Omega}_{k}^{p, *}\right)$ is given by

$$
H\left(\bar{\Omega}_{k}^{p, *}\right)_{\xi}=H^{k, *}\left(\Lambda_{\Delta}^{p} M\right)
$$

where $M$ is the graded $S$-module associated to the symbol of the exterior differential system.

This result is valid for any exterior differential system, and it constitutes the essential step in relating the characteristic cohomology to the symbol of the exterior differential system.

In the unconstrained case the symbol is trivial, and thus as noted above $M$ and the $\Delta$-exterior products are free QFG $S$-modules. This gives $\bar{E}_{1}^{k, q}=0$ for $q<n$, hence $E_{1}^{p, q}=0$ for $p>0, q<n$, and from this the desired result. Q.E.D. 
Corollary. In the local unconstrained case the sequence

$$
0 \rightarrow \bar{H}^{n} \stackrel{d_{1}}{\longrightarrow} E_{1}^{1, n} \stackrel{d_{1}}{\longrightarrow} E_{1}^{2, n} \stackrel{d_{1}}{\longrightarrow} \cdots
$$

is exact.

The groups $E_{1}^{p, n}$ are for $p>0$ the sections of a vector bundle whose fiber at $\xi \in X$ is given by

$$
E_{1, \xi}^{p, n} \cong \Lambda_{\Delta}^{p} M / S^{+} \Lambda_{\Delta}^{p} M
$$

Thus the fiber has as basis any set of free generators of $\Lambda_{\Delta}^{p} M$.

Remark. This corollary may be compared with the contact manifold example in Section 1. The (elementary) argument given there dealt with the unprolonged, unconstrained case with one dependent variable (which is equivalent to $\operatorname{rank}(M)=1$ in the present setting). In that case it identified $\bar{H}_{0}^{n}$ with the range of a second-order linear differential operator. By prolonging, that operator becomes first-order linear on a suitable jet bundle-it is just the Euler-Lagrange operator $d_{1}: \bar{H}^{n} \rightarrow E_{1}^{1, n}$ in the present setting. Moreover, the sequence in the corollary gives an exact complex with the Euler-Lagrange operator as the initial term.

The above theorem, together with the corollary, contains completely the classical formalism of Euler-Lagrange operators, Helmholtz operators, and so forth. Moreover, it also contains an "explanation" of the various complicated classical formulae as simply reflecting choices of generators for certain free QFG modules and then expressing other elements in terms of these generators. We shall now explain this. In order to keep the notation as simple as possible, we shall do this in the case of one independent variable $x$ and one dependent variable $u$.

We thus set $u_{0}=u$ and let

$$
\left(x, u_{0}, u_{1}, u_{2}, \ldots\right)
$$

denote the standard coordinates in $J^{\infty}(\mathbb{R}, \mathbb{R})$, with the contact system having generators

$$
\begin{aligned}
\theta=\theta_{0} & =d u_{0}-u_{1} d x, \\
\theta_{1} & =d u_{1}-u_{2} d x,
\end{aligned}
$$

and structure equations

$$
d \theta_{k}=-\theta_{k+1} \wedge d x .
$$

In this case $M \cong \mathbb{F}[x]$ and the statements that we have free generators

for $\mathbb{F}[x]$, for $\Lambda_{\Delta}^{2} \mathbb{F}[x]$,

translate into the statements that the closed differential forms 
(o) $d x$,

(i) $\theta_{0} \wedge d x$,

(ii) $\theta_{2 k+1} \wedge \theta_{0} \wedge d x$,

give a framing for $\bar{H}^{1}, E_{1}^{1,1}, E_{1}^{2,1}, \ldots$.

A class $[\lambda] \in \bar{H}^{1}$ is represented by a 1 -form

$$
\lambda=f\left(x, u, u_{1}, \ldots, u_{k}\right) d x
$$

and corresponds to a functional

$$
I_{\lambda}(u)=\int f\left(x, u(x), u^{\prime}(x), \ldots, u^{(k)}(x)\right) d x
$$

which, for $u$ with compact support, depends only on the class [ $\lambda]$ in $\bar{H}^{1}$. As explained in Section 1.4, the Euler-Lagrange equations of this functional are

$$
d_{1}[\lambda]=0 \text {. }
$$

By what we have said above, we will have

$$
[d \lambda]=\left[d_{V} \lambda\right]=\left[E(\lambda) \theta_{0} \wedge d x\right]
$$

for some unique operator $E(\lambda)$, which is of course the Euler-Lagrange operator. To determine $E(\lambda)$ we let $\equiv$ denote congruence modulo exact forms, and using the notations and structure equations from Section 2.1, we have

$$
\begin{aligned}
d_{1}[\lambda]= & {\left[d_{V} \lambda\right] } \\
d_{V} \lambda= & \left(\frac{\partial f}{\partial u_{0}} \theta_{0}+\frac{\partial f}{\partial u_{1}} \theta_{1}+\frac{\partial f}{\partial u_{2}} \theta_{2}+\cdots\right) \wedge d x \\
= & \frac{\partial f}{\partial u_{0}} \theta_{0} \wedge d x-\frac{\partial f}{\partial u_{1}} d \theta_{0}-\frac{\partial f}{\partial u_{2}} d \theta_{1}-\cdots \\
\equiv & \frac{\partial f}{\partial u_{0}} \theta_{0} \wedge d x-D\left(\frac{\partial f}{\partial u_{1}}\right) \theta_{0} \wedge d x-D\left(\frac{\partial f}{\partial u_{2}}\right) \theta_{1} \wedge d x-\cdots \\
= & \left(\frac{\partial f}{\partial u_{0}}-D\left(\frac{\partial f}{\partial u_{1}}\right)\right) \theta_{0} \wedge d x+D\left(\frac{\partial f}{\partial u_{2}}\right) d \theta_{0}+\cdots \\
\equiv & \left(\frac{\partial f}{\partial u_{0}}-D\left(\frac{\partial f}{\partial u_{1}}\right)+D^{2}\left(\frac{\partial f}{\partial u_{2}}\right)\right) \theta_{0} \wedge d x-\cdots \\
& \vdots \\
\equiv & {\left[\frac{\partial f}{\partial u_{0}}-D\left(\frac{\partial f}{\partial u_{1}}\right)+D^{2}\left(\frac{\partial f}{\partial u_{2}}\right)+\cdots+(-1)^{k} D^{k}\left(\frac{\partial f}{\partial u_{k}}\right)\right] \theta_{0} \wedge d x }
\end{aligned}
$$

where $f=f\left(x, u, u_{1}, \ldots, u_{k}\right)$. The operator in the brackets is the classical Euler-Lagrange operator $E(\lambda)$. The formula for $E(\lambda)$ is simply a reflection of how one expresses $x^{k}, k>0$, in terms of the generator $1 \in \mathbb{F}[x]$. 
We note that if $k$ is the largest integer such that $\frac{\partial f}{\partial u_{k}} \neq 0$, then $D^{k}\left(\frac{\partial f}{\partial u_{k}}\right)=\left(\frac{\partial^{2} f}{\partial u_{k}^{2}}\right) u_{2 k}+$ (terms depending on $\left.\left(x, u_{0}, u_{1}, \ldots, u_{2 k-1}\right)\right)$.

Thus, unless $f$ is linear in $u_{k}$, the Euler-Lagrange operator is of even order $2 k$.

We now consider a class $\left[E \theta_{0} \wedge d x\right] \in E_{1}^{1,1}$ where $E=E\left(x, u, u_{1}, \ldots, u_{k}\right)$ and ask when $E$ is of the form $E(\lambda)$ for some $\lambda=f d x$. Again this depends only on the class in $E_{1}^{1,1}$ of $E \theta_{0} \wedge d x$, and by the above corollary the condition is $d_{1}\left[E \theta_{0} \wedge d x\right]=0$.

By what was said above we will have

$$
\left[d_{V}\left(E \theta_{0} \wedge d x\right)\right]=\left[\sum_{k \geqq 0} H_{2 k+1}(E) \theta_{2 k+1} \wedge \theta_{0} \wedge d x\right]
$$

for unique operators $H_{2 k+1}(E)$. The fact that only odd orders appear is a reflection of the fact noted above that in general the Euler-Lagrange operators are of even order. Thus, if $E=E\left(x, u_{0}, u_{1}, \ldots, u_{2 k+1}\right)$ with $\partial E / \partial u_{2 k+1} \neq$ 0 , then $d_{V}\left(E \theta_{0} \wedge d x\right) \equiv \partial E / \partial u_{2 k+1} \theta_{2 k+1} \wedge \theta_{0^{\wedge}} d x \bmod \left\{\theta_{2 k-1} \wedge \theta_{0}, \ldots, \theta_{1} \wedge \theta_{0}\right\}$, so $d_{1}\left[E \theta_{0} \wedge d x\right] \neq 0$ in this case. These operators will express the Helmholtz conditions corresponding to our particular choice of free generators $x^{2 k+1} \wedge 1$ for $\Lambda_{\Delta}^{2} \mathbb{F}[x]$, and determining them explicitly will simply reflect how one expresses $x^{2 k} \wedge 1$ in terms of these generators. Explicitly, we have

$$
\begin{aligned}
d_{V}\left(E \theta_{0} \wedge d x\right) & =\left(\frac{\partial E}{\partial u_{1}} \theta_{1}+\frac{\partial E}{\partial u_{2}} \theta_{2}+\frac{\partial E}{\partial u_{3}} \theta_{3}+\frac{\partial E}{\partial u_{4}} \theta_{4}+\cdots\right) \wedge \theta_{0} \wedge d x \\
\frac{\partial E}{\partial u_{2}} \theta_{2} \wedge \theta_{0} \wedge d x & =\frac{\partial E}{\partial u_{2}} d\left(\theta_{1} \wedge \theta_{0}\right) \equiv-D\left(\frac{\partial E}{\partial u_{2}}\right) \theta_{1} \wedge \theta_{0} \wedge d x \\
\frac{\partial E}{\partial u_{4}} \theta_{4} \wedge \theta_{0} \wedge d x & =\frac{\partial E}{\partial u_{4}} d\left(\theta_{3} \wedge \theta_{0}\right)-\frac{\partial E}{\partial u_{4}} \theta_{3} \wedge \theta_{1} \wedge d x \\
& \equiv-D\left(\frac{\partial E}{\partial u_{4}}\right) \theta_{3} \wedge \theta_{0} \wedge d x-\left(\frac{\partial E}{\partial u_{4}}\right) d\left(\theta_{2} \wedge \theta_{1}\right) \\
& \equiv-D\left(\frac{\partial E}{\partial u_{4}}\right) \theta_{3} \wedge \theta_{0} \wedge d x+D\left(\frac{\partial E}{\partial u_{4}}\right) \theta_{2} \wedge \theta_{1} \wedge d x \\
& \equiv-2 D\left(\frac{\partial E}{\partial u_{4}}\right) \theta_{3} \wedge \theta_{0} \wedge d x+D\left(\frac{\partial E}{\partial u_{4}}\right) d\left(\theta_{2} \wedge \theta_{0}\right) \\
& \equiv-2 D\left(\frac{\partial E}{\partial u_{4}}\right) \theta_{3} \wedge \theta_{0} \wedge d x-D^{2}\left(\frac{\partial E}{\partial u_{4}}\right) \theta_{2} \wedge \theta_{0} \wedge d x \\
& \equiv-2 D\left(\frac{\partial E}{\partial u_{4}}\right) \theta_{3} \wedge \theta_{0} \wedge d x-D^{2}\left(\frac{\partial E}{\partial u_{4}}\right) d\left(\theta_{1} \wedge \theta_{0}\right) \\
& \equiv-2 D\left(\frac{\partial E}{\partial u_{4}}\right) \theta_{3} \wedge \theta_{0} \wedge d x+D^{3}\left(\frac{\partial E}{\partial u_{4}}\right) \theta_{1} \wedge \theta_{0} \wedge d x
\end{aligned}
$$


In general, if we write

$$
x^{2 k} \wedge 1=\sum_{\ell \geqq 0} C_{\ell}^{k} x^{2 k-2 \ell-1} \cdot\left(x^{2 \ell+1} \wedge 1\right),
$$

then

$$
H_{2 \ell+1}(E)=\frac{\partial E}{\partial u_{2 \ell+1}}-\sum_{k} C_{\ell}^{k} D^{2 k-2 \ell-1}\left(\frac{\partial E}{\partial u_{2 k}}\right) .
$$

This shows, in the most direct manner, how the $\Delta$-exterior algebra results directly translate into classical formulae, in this case, the Helmholtz conditions $H_{2 \ell+1}(E)=0$ which are the necessary and sufficient conditions that an expression $E$ be of the form $E=E(\lambda)$ for some functional $I_{\lambda}$. A different choice of generators for the module $\Lambda_{\Delta}^{2} \mathbb{F}[x]$ would yield a different, albeit equivalent, set of equations.

4.2. Involutivity and $\Delta$-exterior powers. Let $M$ be a QFG graded $S$-module with minimal resolution

$$
0 \rightarrow F_{\ell} \stackrel{\varphi_{\ell}}{\longrightarrow} F_{\ell-1} \stackrel{\varphi_{\ell-1}}{\longrightarrow} \cdots \rightarrow F_{1} \stackrel{\varphi_{1}}{\longrightarrow} F_{0} \stackrel{\varphi_{0}}{\longrightarrow} M \rightarrow 0
$$

by QFG free graded modules $F_{\nu}$. Recall that $\ell \leqq n$ and that

$$
\begin{aligned}
& \varphi_{0}: F_{0}^{k} \rightarrow M^{k}, \\
& \varphi_{\nu}: F_{\nu}^{k} \rightarrow \bigoplus_{\ell \geqq k+1} F_{\nu-1}^{\ell}, \quad \nu \geqq 1 .
\end{aligned}
$$

The first of these simply states that $\varphi_{0}$ maps free generators of $F_{0}$ onto a set of generators of $M$, while the second says that we have chosen a minimal set of free generators of $F_{0}$ with the property, that we then choose a minimal set of generators for the relations among the generators of $M$, and so forth.

Definition. (i) $M$ is involutive if each $\varphi_{\nu}, \nu \geqq 1$, has graded degree exactly equal to one.

(ii) The integer $\ell$ giving the length of the resolution (1) will be called the characteristic number of the module $M$.

Intuitively, $M$ is involutive if the relations among a minimal set of generators of $M$ are themselves minimally generated by relations in degree one, if the relations among the relations are themselves minimally generated in degree one, and so forth. If $M$ is generated in degree zero, then involutivity is equivalent to

$$
H^{+, q}(M)=0
$$

where

$$
H^{q}(M)=\bigoplus_{k} H^{k, q}(M)
$$

is the graded vector space of Spencer cohomology. Whether or not $M$ is generated in degree zero, the characteristic number $\ell(M)$ is defined by

$$
\ell(M)=\min \left\{\ell \mid H^{q}(M)=0 \text { for } 0 \leqq q<n-\ell\right\} .
$$


Below we shall recall the definition of the Cartan characters $s_{0}, s_{1}, \ldots, s_{n}$ of the first prolongation of an exterior differential system. These integers satisfy

$$
s_{0} \geqq s_{1} \geqq \cdots \geqq s_{n}
$$

and are easily computed in practice. Setting

$$
\sigma_{i}=s_{i}-s_{i+1}, \quad i=0,1, \ldots, n-1,
$$

and $\sigma_{n}=s_{n}$, the characteristic number $\ell \geq 0$ is also uniquely characterized as the smallest integer so that

$$
\sigma_{n-\ell} \neq 0
$$

and this is how it is computed in geometric examples. We remark that although the Cartan characters are not invariant under prolongation, the characteristic number defined by (2) is invariant.

Our main algebraic result is given by the

Theorem 1. If $M$ is involutive, then so are the $\Delta$-exterior powers $\Lambda_{\Delta}^{p} M$. Moreover,

$$
\ell\left(\Lambda_{\Delta}^{p} M\right) \leqq \ell(M) .
$$

Actually, as our proof will show, the inequality $\ell\left(\Lambda_{\Delta}^{p} M\right) \leqq \ell(M)$ holds without the assumption of involutivity and, once formulated and set up, this result by itself is relatively easy.

It is more substantial to understand the minimal resolution of $\Lambda_{\Delta}^{p} M$ in terms of that of $M$. As we will see below, if there are "sufficiently many" variables and if the symbol is generic among involutive symbols, then $\Lambda_{\Delta}^{2} M$ will be freely generated in low degrees but will have relations in higher degrees. It is perhaps for this reason that our proof of the theorem stated above is not intrinsic.

A geometric application results from the fact that

$$
H^{q}\left(\Lambda_{\Delta}^{p} M\right)=0, \quad 0 \leqq q<n-\ell .
$$

Before proving Theorem 1, we shall make a few preliminary remarks. For these we will let $\mathscr{F}_{0}$ be an exterior differential system on a manifold $X_{0}$ and $E_{0} \subset T_{p} X_{0}$ an $n$-dimensional integral element of $\mathscr{I}_{0}$. The concept of what it means for $E_{0}$ to be ordinary may be defined (see pages 73-ff in [2]), and $\mathscr{F}_{0}$ will be said to be involutive in a neighborhood of an ordinary integral element (if such exists). If $E_{0}$ is ordinary, then the symbol is involutive in a neighborhood $U$ of $E_{0}$ in $G_{n}\left(\mathscr{I}_{0}\right)$, and moreover the prolongation tower will satisfy the regularity assumption of Section 1.3 over $U$. Here, it will be convenient to set $X_{0}=U$ and begin the construction of the prolongation tower with $X_{1}$, and when this is done we will say that we are in the local, involutive case.

Finally, we remark that in practice there is a very effective criterion called Cartan's test that allows us to determine when an integral element $E_{0}$ is ordinary in terms of the Cartan characters $s_{1}, s_{2}, \ldots, s_{n}$ mentioned above. We shall review this at an appropriate time. The upshot is that in practice both the issue of involutivity and the characteristic number may be effectively determined from the characters $s_{i}$.

The following application of the theorem follows immediately from the same argument as in the preceding section. 
Theorem 2. In the local, involutive case the characteristic cohomology satisfies

$$
\bar{H}^{q}=0 \text { for } 0<q<n-\ell
$$

where $\ell$ is the characteristic number of the symbol of the exterior differential system.

In the context of fibered manifolds what essentially amounts to the case $\ell=1$ of this result is due to Vinogradov [13]; he refers to it as the two-line theorem. An alternative proof of Vinogradov's result is proposed in [11], but we are unable to follow the argument. Generalizations of Vinogradov's theorem are also given in [1] and [12]. All of these works deal with the situation of fibered manifolds and as so formulated do not imply the above result.

In light of the above result, attention is naturally focussed on the first possibly non-vanishing cohomology groups.

Definition. In the local, involutive case we set $\mathscr{C}=\bar{H}^{n-\ell}$ and shall call $\mathscr{C}$ the group of conservation laws of the exterior differential system.

Much of our subsequent work will be devoted to studying the group of conservation laws in classes of examples and to geometric applications of those results. From the characteristic spectral sequence we have

$$
\mathscr{C} \cong \operatorname{ker} d_{1}: E_{1}^{1, n-\ell} \rightarrow E_{1}^{2, n-\ell} .
$$

This rather abstract looking result will in practice turn out to be extremely important. In effect, this will allow us to systematically study the conservation laws in terms of the symbol, then the subprincipal symbol and so forth. Moreover, when written out, (4) will give the conservation laws as the kernel of a certain canonical linear differential operator and not as a quotient spaceone may think in effect of having an intrinsic harmonic operator for ordinary deRham cohomology. This puts in a general context the phenomena noted in the examples in Section 1.1.

4.3. The tableau and its normal form. We will begin the proof of Theorem 1 from the preceding section in some special cases. As mentioned there, our proof is not intrinsic but will give more than just the statement of the theorem. However, we can explain why the estimate $\ell\left(\Lambda_{\Delta}^{p} M\right) \leqq \ell(M)$ should be true, as follows: "The characteristic number of $M$ is the smallest integer $\ell$ such that if we restrict to a generic $n-\ell$ plane, then $M$ is free. By Proposition 2 in Section 3.2, the restriction of $\Lambda_{\Delta}^{p} M$ to this $n-\ell$ plane will also be free, hence the result." This reasoning is not correct, but it is also not entirely incorrect and captures the essence of the result.

We now recall the device known as a "tableau" introduced by Cartan and used by him to put a symbol in a sort of normal form (further details may be found in pages $141 \mathrm{ff}$ in [2]). Let $W$ and $E$ be vector spaces, $B \subset W^{*} \otimes E$ a subspace, and $B \subset W^{*} \otimes S E$ the submodule generated by $B$. Then $B$ is a symbol and $M=\dot{W}^{*} \otimes S E / B$ is the graded $S$-module associated to $B$.

We may think of $B$ as corresponding to a linear, homogeneous constant coefficient PDE system whose formal power series solutions are given by the graded subspace

$$
A^{\bullet} \subset W \otimes S E^{*}
$$


annihilated by $B$. Thus $A^{\cdot}=\bigoplus_{k \geqq 0} A^{k}$ where $A^{k} \subset W \otimes S^{k} E^{*}$ are the $W$ valued homogeneous polynomials $\overline{\bar{P}}$ of degree $k$ that satisfy

$$
B \cdot P=0
$$

where $B \cdot P \in S^{k-1} E^{*}$. We shall call $A=A^{1}$ the tableau associated to $B$ and shall think of elements $L \in A$ as $W$-valued linear functions on the vector space $E$. Choosing a generic linear coordinate system $x^{1}, \ldots, x^{n}$ we may then ask how many elements $L \in A$ are uniquely determined by their restrictions to linear subspaces $x^{k+1}=\cdots=x^{n}=0$ and whether the restriction of $L$ to this subspace may be arbitrarily prescribed.

To visualize this we let $w^{1}, \ldots, w^{s} \in W^{*}$ be a basis, to be adjusted during the following construction, and $e_{1}, \ldots, e_{n} \in E$ the basis dual to the generic linear coordinate system $x^{1}, \ldots, x^{n}$, which is fixed once and for all. We denote by

$$
b^{\lambda}=b_{\alpha}^{\lambda i} w^{\alpha} \otimes e_{i} \in W^{*} \otimes E
$$

a basis for $B$ and introduce the spanning elements $\theta_{i}^{\alpha}$ for the dual space $A^{*}$ given by projecting $w^{\alpha} \otimes e_{i}$ to $M^{1}=A^{*}$. Then the $\theta_{i}^{\alpha}$ are subject to the defining relations

$$
b_{\alpha}^{\lambda i} \theta_{i}^{\alpha}=0
$$

We then introduce the tableau matrix

$$
\boldsymbol{\theta}=\left\|\begin{array}{cccc}
\theta_{1}^{1} & \theta_{2}^{1} & \cdots & \theta_{n}^{1} \\
\vdots & \vdots & & \vdots \\
\theta_{1}^{s} & \theta_{1}^{s} & \cdots & \theta_{n}^{s}
\end{array}\right\| .
$$

By definition, the Cartan characters $s_{0}, s_{1}, s_{2}, \ldots, s_{n}$ are given by $s_{0}=s$ and

$$
s_{1}+\cdots+s_{k}=\left\{\begin{array}{l}
\text { number of independent } 1 \text {-forms } \\
\theta_{i}^{\alpha} \text { in the first } k \text { columns of } \boldsymbol{\theta}
\end{array}\right\} .
$$

We first choose our basis $w^{\alpha}$ for $W^{*}$ so that $\theta_{1}^{1}, \ldots, \theta_{1}^{s_{1}}$ are linearly independent, we then refine that choice so that $\theta_{1}^{1}, \ldots, \theta_{1}^{s_{1}}, \theta_{2}^{1}, \ldots, \theta_{2}^{s_{2}}$ are linearly independent, and so forth (see page 141 of [2] for further discussion). Then $s_{1} \geqq s_{2} \geqq \cdots \geqq s_{n}$ and $s_{1}+\cdots+s_{n}=\operatorname{dim} A$. We may picture the normalized tableau matrix as looking like

$$
\left\|\begin{array}{cccc}
\theta_{1}^{1} & \theta_{2}^{1} & & \theta_{n}^{1} \\
\vdots & \vdots & \cdots & \theta_{n}^{s_{n}} \\
& & & * \\
& \theta_{2}^{s_{2}} & & * \\
\theta_{1}^{s_{1}} & * & & * \\
* & * & & *
\end{array}\right\|
$$

where for $\beta>s_{k}$ the form $\theta_{k}^{\beta}$ is a linear combination of the other $\theta_{i}^{\alpha}$ where $i \leqq k$ and $\alpha \leqq s_{i}$, i.e., the $*$ forms are linear combinations of the other $\theta$ 's. 
Actually, for the purposes of the argument we shall be giving, it will be advantageous to organize the normalized tableau matrix into blocks

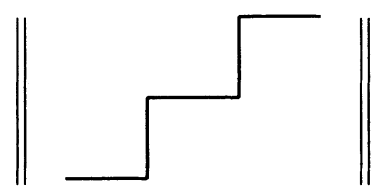

where the $s_{i}$ are constant in each block. We will develop the proof of our result through examination of a number of special cases. Before turning to this we will introduce some notation.

- $d x^{i}$ and $\theta_{I}^{\alpha}$ are linear functions on two vector spaces, the $d x^{i}$ being linearly independent and the $\theta_{I}^{\alpha}$ subject to the relations

$$
b_{\alpha}^{\lambda i} \theta_{I i}^{\alpha}=0 \text {. }
$$

- $\Omega^{p, q}$ is spanned by the forms

$$
\theta_{I_{1}}^{\alpha_{1}} \wedge \cdots \wedge \theta_{I_{p}}^{\alpha_{p}} \wedge d x^{j_{1}} \wedge \cdots \wedge d x^{j_{q}}
$$

and $\Omega^{*}=\bigoplus \Omega^{p, q}$.

- The differential

is defined by $d\left(d x^{i}\right)=0$ and

$$
d: \Omega^{p, q} \rightarrow \Omega^{p, q+1}
$$

$$
d \theta_{I}^{\alpha}=-\theta_{I i}^{\alpha} \wedge d x^{i},
$$

together with the usual properties of being a derivation of the exterior algebra.

- We will denote by $\Lambda_{m}^{*}=\bigoplus \Lambda_{m}^{p, q}$ a complex analogous to $\Omega^{p, q}$ but where the number of $d x^{i}$ 's is $m$ and where there are no relations on the $\theta_{I}^{\alpha}$-this "free" case models the unconstrained geometric situation in which the number of independent variables is $m$, and we have seen that

$$
H^{k}\left(\Lambda_{m}^{*}\right)=0, \quad k<m
$$

4.4. The determined case. By definition, the determined case is an exterior differential system whose Cartan characters are the same as for a determined PDE system

$$
\partial u / \partial x^{n}=\left\{\begin{array}{l}
\text { constant linear combination } \\
\text { of } \partial u / \partial x^{1}, \ldots, \partial u / \partial x^{n-1}
\end{array}\right.
$$

where $u={ }^{t}\left(u^{1}, \ldots, u^{s}\right)$. The Cartan characters are given by

$$
s_{1}=\cdots=s_{n-1}=s, s_{n}=0 .
$$

In this case we may solve for the last column $\underline{\theta}_{n}$ of the tableau matrix in terms of the first $n-1$ columns $\underline{\theta}_{\rho}, 1 \leqq \rho \leqq n-1$. Thus we have relations

$$
\underline{\boldsymbol{\theta}}_{n}=b^{\rho} \underline{\boldsymbol{\theta}}_{\rho}
$$


where each $b^{\rho}$ is an $s \times s$ matrix. These relations induce relations

$$
\underline{\boldsymbol{\theta}}_{I n}=b^{\rho} \underline{\boldsymbol{\theta}}_{I_{\rho}}
$$

on the vector-valued forms $\underline{\boldsymbol{\theta}}_{J}={ }^{t}\left(\ldots, \theta_{J}^{\alpha}, \ldots\right)$. When written out fully, these relations are

$$
\begin{gathered}
\theta_{n}^{\alpha}=b_{\beta}^{\alpha \rho} \theta_{\rho}^{\beta}, \\
\theta_{I n}^{\alpha}=b_{\beta}^{\alpha \rho} \theta_{I \rho}^{\beta} .
\end{gathered}
$$

We shall refer to the case $b^{\rho}=0$ as the split case; on the symbol level it looks like the PDE system $\partial u / \partial x^{n}=0$. The opposite extreme is when the $b^{\rho}$ are generic.

It is well known and easy to prove that any determined exterior differential system is involutive. is

The minimal free resolution of the graded $S$-module associated to the symbol

$$
0 \rightarrow W_{1, .}^{*} \stackrel{b}{\longrightarrow} W^{*} \rightarrow M \rightarrow 0
$$

where $W^{*}, W_{1}^{*}$ are $s$-dimensional vector spaces with respective bases $w^{\alpha}, w_{1}^{\alpha}$ and where

$$
b\left(w_{1}^{\alpha}\right)=e_{n} w^{\alpha}-b_{\beta}^{\alpha \rho} e_{\rho} \cdot w^{\beta}
$$

corresponding to (1). We want to infer properties of the minimal free resolution of the $\Lambda_{\Delta}^{p} M_{s}$. For this we will use the standard shift notation $P[\ell]$ for a graded object $P$-thus $P=\bigoplus_{k} P^{k}$ and $P[\ell]=\bigoplus_{k} P[\ell]^{k}$ where $P[\ell]^{k}=P^{k+\ell}$. We introduce the complexes

$$
\begin{aligned}
\Omega^{p, q} & =d x^{n} \wedge \Omega^{\prime p, q-1} \cong \Lambda_{n-1}^{p, q-1}[1], \\
\Omega^{\prime p, q} & =\Omega^{p, q} / \Omega^{p, q} \cong \Lambda_{n-1}^{p, q} .
\end{aligned}
$$

The first line means that $\Omega^{\prime *}$ is a subcomplex of $\Omega^{*}$ which is isomorphic as a complex to $\Lambda_{n-1}^{*}[1]$, this being the case since there are no relations among the $\theta_{\rho_{1} \ldots \rho_{k}}^{\alpha}$. In the second line, $\Omega^{\prime \prime *}$ is in effect obtained by setting $d x^{n}=0$, and it is again isomorphic as a complex to $\Lambda_{n-1}^{*}$. From the exact cohomology sequence of

$$
0 \rightarrow \Omega^{\prime *} \rightarrow \Omega^{*} \rightarrow \Omega^{\prime *} \rightarrow 0
$$

together with (1) in the preceding section, we obtain

$$
H^{p, q}\left(\Omega^{*}\right)=0, \quad q \leqq n-2,
$$

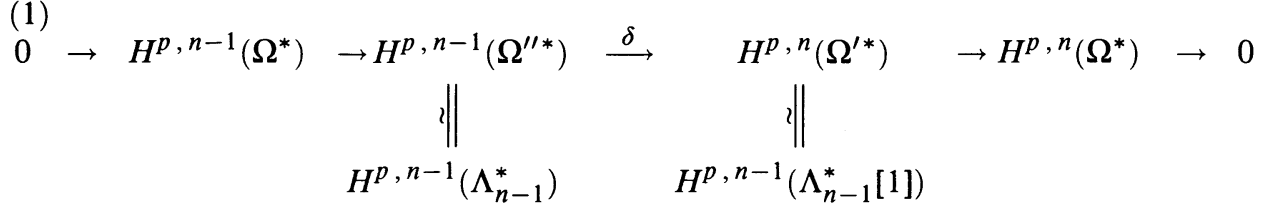

The first result implies (3) in Theorem 1 in Section 4.2, which as one sees is relatively elementary once one sets the problem up and has the statement that the $\Lambda_{\Delta}^{p} M$ are free in case $M$ is. 
The exact sequence (1) will now be examined, and we shall see a great difference between the cases $p=1$ and $p \geqq 2$. In the former we will see that $\delta=0$, just as if we were in the split case; in the latter almost the opposite will hold.

We first compute $\delta$ in the case $p=1$. Setting $d x_{i}=(-1)^{i-1} d x^{1} \wedge \cdots \wedge$ $\widehat{d x^{i}} \wedge \cdots \wedge d x^{n}, d x^{\prime}=d x_{n}, d x_{\rho}^{\prime}=(-1)^{\rho} d x^{1} \wedge \cdots \wedge \widehat{d x^{\rho}} \wedge \cdots \wedge d x^{n-1}$ and $d x=$ $d x^{1} \wedge \cdots \wedge d x^{n}$ we have bases

$$
\begin{array}{cll}
\theta^{\alpha} \wedge d x^{\prime} & \text { for } & H^{1, n-1}\left(\Omega^{\prime * *}\right), \\
\theta^{\alpha} \wedge d x & \text { for } & H^{1, n}\left(\Omega^{\prime *}\right) \quad\left(d x=d x^{n} \wedge d x^{\prime}\right) .
\end{array}
$$

Now

$$
\begin{aligned}
\delta\left(\theta^{\alpha} \wedge d x^{\prime}\right) & =\text { class of } d\left(\theta^{\alpha} \wedge d x^{\prime}\right) \\
& =\text { class of }-\theta_{n}^{\alpha} \wedge d x \\
& =\text { class of } d\left(b_{\beta}^{\alpha \rho} \theta^{\beta} \wedge d x_{\rho}\right), \quad 1 \leqq \rho \leqq n-1, \\
& =\text { class of }(-1)^{n-1} d x^{n} \wedge d\left(b_{\beta}^{\alpha \rho} \theta^{\beta} \wedge d x_{\rho}^{\prime}\right) \\
& =0 .
\end{aligned}
$$

Thus when $p=1$ there is no difference between the split and the general case.

We next turn to the case $p=2$. Let $E^{\prime}$ be the subspace $d x^{n}=0$ of $E$ with basis $e_{1}, \ldots, e_{n-1}, S^{\prime}=S E^{\prime}$ the symmetric algebra of $E^{\prime}$, and $M^{\prime}=$ $W^{\prime *}=W^{*} \otimes_{\mathbb{F}} S^{\prime}$. Then as we have seen at the end of Section 3.2, $\Lambda_{\Delta}^{2} M^{\prime}$ has generators

$$
e_{I} w^{\alpha} \cdot \wedge w^{\beta}
$$

where $I=\left(\rho_{1}, \ldots, \rho_{k}\right)$ and relations

$$
e_{I} w^{\alpha} \wedge w^{\beta}+(-1)^{k} e_{I} w^{\beta} \wedge w^{\alpha} \equiv 0
$$

among the generators, where $\equiv$ is congruence modulo the action of the maximal ideal $S^{\prime+}$ of $S E^{\prime}$. Thus we shall write elements of $V^{\prime}=: \Lambda_{\Delta}^{2} M^{\prime} / S^{+} \cdot \Lambda_{\Delta}^{2} M^{\prime}$ as

$$
\varphi=\varphi_{\alpha \beta}^{I} e_{I} w^{\alpha} \wedge w^{\beta}, \quad \varphi+(-1)^{|I| t} \varphi=0
$$

where $\left({ }^{t} \varphi\right)_{\alpha \beta}^{I}=\varphi_{\beta \alpha}^{I}$. Now each of $H^{2, n-1}\left(\Omega^{\prime \prime *}\right)$ and $H^{2, n}\left(\Omega^{\prime *}\right)$ is isomorphic to $V^{\prime}$, and making this identification we shall compute $\delta: V^{\prime} \rightarrow V^{\prime}$. For this we introduce indeterminates $\xi_{1}, \ldots, \xi_{n-1}$ and set

$$
\begin{aligned}
& \varphi(\xi)=\left\|\varphi_{\alpha \beta}(\xi)\right\|=\left\|\varphi_{\alpha \beta}^{I} \xi_{I}\right\|, \\
& b(\xi)=\left\|b_{\beta}^{\alpha \rho} \xi_{\rho}\right\| .
\end{aligned}
$$

Proposition. With the above notations

Proof. We set

$$
(\delta \varphi)(\xi)=[\varphi(\xi), b(\xi)] \quad\left(={ }^{t} b(\xi) \varphi(\xi)-\varphi(\xi) b(\xi)\right) .
$$

$$
\underline{\varphi}=\varphi_{\alpha \beta}^{I} \theta_{I}^{\alpha} \wedge \theta^{\beta} \wedge d x^{\prime} \in H^{2, n-1}\left(\Omega^{\prime \prime *}\right) \cong V^{\prime}
$$


and must compute

$$
\delta(\underline{\varphi})=\text { class of } d \varphi \in H^{2, n}\left(\Omega^{\prime *}\right) \cong V^{\prime} .
$$

Now we have (note the signs)

$$
\begin{aligned}
d\left(\theta_{I}^{\alpha} \wedge \theta^{\beta} \wedge d x^{\prime}\right) & =\theta_{I n}^{\alpha} \wedge \theta^{\beta} \wedge d x+\theta_{I}^{\alpha} \wedge \theta_{n}^{\beta} \wedge d x \\
& =\left(b_{\gamma}^{\alpha \rho} \theta_{I \rho}^{\gamma} \wedge \theta^{\beta}+b_{\gamma}^{\beta \rho} \theta_{I}^{\alpha} \wedge \theta_{\rho}^{\gamma}\right) \wedge d x \\
& \equiv\left(b_{\gamma}^{\alpha \rho} \theta_{I \rho}^{\gamma} \wedge \theta^{\beta}-b_{\gamma}^{\beta \rho} \theta_{I \rho}^{\alpha} \wedge \theta^{\gamma}\right) \wedge d x
\end{aligned}
$$

where $\equiv$ is congruence modulo exact forms and we have used

$$
d\left(\theta_{I}^{\alpha} \wedge \theta^{\gamma} \wedge d x_{\rho}\right)=\left(\theta_{I \rho}^{\alpha} \wedge \theta^{\gamma}+\theta_{I}^{\alpha} \wedge \theta_{\rho}^{\gamma}\right) \wedge d x .
$$

The proposition follows by using the symmetry conditions (2) on $\varphi$. Q.E.D.

Discussion. $M$ has a two-step minimal free resolution with minimal generators and minimal generating relations given respectively by

$$
\begin{aligned}
& w^{\alpha}, \\
& e_{n} w^{\alpha}-b_{\beta}^{\alpha \rho} e_{\rho} \cdot w^{\beta}=0 .
\end{aligned}
$$

Using the proposition, we will show that $\Lambda_{\Delta}^{2} M$ has a minimal free resolution

$$
0 \rightarrow F_{1} \stackrel{\lambda}{\rightarrow} F_{0} \rightarrow \Lambda_{\Delta}^{2} M \rightarrow 0
$$

where $F_{1} \neq 0$ and $\lambda$ has degree one. It follows that:

$$
\Lambda_{\Delta}^{2} \text { is involutive and } \ell\left(\Lambda_{\Delta}^{2} M\right)=\ell(M)
$$

We will also see that in the split case $F_{1}$ has generators in all degrees where $F_{0}$ does, but in the generic case generators of $F_{1}$ appear only in degrees that are high relative to $s$ and $n$; i.e., in low degrees the generators of $\Lambda_{\Delta}^{2} M$ generate free summands.

In order to best understand the situation, we will work through the first few degrees, and from these draw general conclusions based on the evident patterns that will emerge.

Degree zero. Minimal generators and relations among the generators are

$$
\begin{aligned}
& w^{\alpha} \wedge w^{\beta}, \quad \alpha<\beta, \\
& e_{n}\left(\varphi_{\alpha \beta} w^{\alpha} \wedge w^{\beta}\right)-e_{\rho} \cdot\left(\varphi_{\alpha \gamma} b_{\beta}^{\gamma \rho} w^{\alpha} \wedge w^{\beta}\right)=0 \text { where } \\
& \varphi+{ }^{t} \varphi=0,\left[\varphi, b^{\rho}\right]=0 \text { for } \rho=1, \ldots, n-1
\end{aligned}
$$

Thus, when we try to "lift" the relations (ii) from $M$ to $\Lambda_{\Delta}^{2} M$ we are only able to lift certain linear combinations (ii) 0 , namely those which satisfy the equations (ii) ${ }_{0}^{\prime}$.

Degree one. Minimal generators are a linearly independent set of elements

$$
\psi=\psi_{\alpha \beta}^{\rho} e_{\rho} \cdot w^{\alpha} \wedge w^{\beta}, \quad \psi-{ }^{t} \psi=0
$$


taken modulo those of the form
(i) ${ }_{1}^{\prime}$
$\psi^{\rho}=\left[\varphi, b^{\rho}\right]$,
$\varphi+{ }^{t} \varphi=0$.

Relations among these generators are given by

$$
e_{n} \cdot\left(\psi_{\alpha \beta}^{\rho} e_{\rho} \cdot w^{\alpha} \wedge w^{\beta}\right)-e_{\sigma} \cdot\left(\psi_{\alpha \gamma}^{\rho} b_{\beta}^{\gamma \sigma} e_{\rho} \cdot w^{\alpha} \wedge w^{\beta}\right)=0
$$

where

(ii) ${ }_{1}^{\prime}$

$$
\left[b^{\sigma}, \psi^{\rho}\right] e_{\rho} e_{\sigma}=0 .
$$

Thus we find that the "expected" generators (i) ${ }_{1}$ must be corrected by "moding out" by the range (i) ${ }_{1}^{\prime}$ of the operator $\varphi \rightarrow\left[\varphi, b^{\rho}\right]$ whose kernel gave (ii) ${ }_{0}^{\prime}$, and moreover that trying to lift the relations (ii) as in (ii) ${ }_{1}$ requires that the condition (ii) ${ }_{1}^{\prime}$ be satisfied.

Degree two. Minimal generators are a linearly independent set of elements

$$
\psi=\psi_{\alpha \beta}^{\rho \sigma} e_{\rho} e_{\sigma} w^{\alpha} \wedge w^{\beta}, \quad \psi+{ }^{t} \psi=0
$$

taken modulo those of the form

(i) ${ }_{2}^{\prime}$

$$
\psi^{\rho \sigma}=\left[b^{\rho}, \varphi^{\sigma}\right]+\left[b^{\rho}, \varphi^{\sigma}\right], \quad \varphi-{ }^{t} \varphi=0 .
$$

Relations among these generators are

$$
e_{n} \cdot\left(\psi_{\alpha \beta}^{\rho \sigma} e_{\rho} e_{\sigma} w^{\alpha} \wedge w^{\beta}\right)-e_{\tau} \cdot\left(\psi_{\alpha \gamma}^{\rho \sigma} b_{\beta}^{\gamma \tau} e_{\rho} e_{\sigma} w^{\alpha} \wedge w^{\beta}\right)=0
$$

where

(ii) ${ }_{2}^{\prime}$

$$
\left[b^{\rho}, \psi^{\sigma \tau}\right] e_{\rho} e_{\sigma} e_{\tau}=0 .
$$

From these first few terms the general pattern is clear. The statements $(i)_{i}$, (i) ${ }_{i}^{\prime}$, (ii) ${ }_{i}$, (ii) ${ }_{i}^{\prime}$ are interpretations of the exact cohomology sequence with the various identifications being made. As far as the involutivity goes, from (1) it follows that for all $p$ there is a two-step minimal free resolution

$$
0 \rightarrow F_{1, p} \rightarrow F_{0, p} \rightarrow \Lambda_{\Delta}^{p} M \rightarrow 0
$$

where as graded vector spaces

$$
\left\{\begin{array}{l}
F_{0, p} / S^{+} F_{0, p} \cong \operatorname{coker} \delta, \\
F_{1, p} / S^{+} F_{1, p} \cong \operatorname{ker} \delta \\
\operatorname{deg} \delta=1 .
\end{array}\right.
$$

The mapping $\delta$ probes when a linear combination

$$
\varphi=\varphi_{\alpha_{1} \ldots \alpha_{p}}^{I_{1} \ldots I_{p-1}} \theta_{I_{1}}^{\alpha_{1}} \wedge \cdots \wedge \theta_{I_{p-1}}^{\alpha_{p-1}} \wedge \theta^{\alpha_{p}} \wedge d x_{n}, \quad\left|I_{1}\right|+\cdots+\left|I_{p-1}\right|+1=k,
$$

of elements of $H^{p, n-1}\left(\Lambda_{n-1}^{*}\right)$ can be lifted to a closed form

$$
\psi=\varphi+\eta_{\alpha_{1} \ldots \alpha_{p}}^{I_{1} \ldots I_{p-1, \rho}} \theta_{I_{1}}^{\alpha_{1}} \wedge \cdots \wedge \theta_{I_{p-1}}^{\alpha_{p-1}} \wedge \theta^{\alpha_{p}} \wedge d x_{\rho},\left|I_{1}\right|+\cdots+\left|I_{p-1}\right|+1=k,
$$


of the same weight $k-n+1$ as $\varphi$. Writing

$$
0=d \psi=E d x
$$

we see that the formula $\varphi \rightarrow E$ has degree one, i.e., the relations among the generators of $\Lambda_{\Delta}^{p} M$ are themselves generated in degree one.

Finally, again in the case $p=2$ we will make some observations about the operator

$$
\varphi(\xi) \rightarrow[\varphi(\xi), b(\xi)] .
$$

If, say, degree $\varphi(\xi)=2 k-1$ is odd, then this is an operator from a vector space of dimension $\left(\begin{array}{c}n-2+2 k \\ 2 k-1\end{array}\right) \frac{s(s+1)}{2}$ to a vector space of dimension $\left(\begin{array}{c}n-1+2 k \\ 2 k\end{array}\right) \frac{s(s-1)}{2}$. The inequality

$$
\left(\begin{array}{c}
n-1+2 k \\
2 k
\end{array}\right) \frac{s(s-1)}{2} \geqq\left(\begin{array}{c}
n-2+2 k \\
2 k-1
\end{array}\right) \frac{s(s+1)}{2}
$$

is for $s \geqq 2$ equivalent to

$$
1+\frac{n-1}{2 k} \geqq 1+\frac{2}{s-1} .
$$

For fixed $s$ and $n$ this inequality is satisfied for

$$
k \leqq(s-1)(n-1) / 4
$$

but not for $k>(s-1)(n-1) / 4$. Thus we may expect that for a generic symbol the operator (4) will be injective for small $k$ but not for large $k$. This means that in the minimal free resolution (3) the minimal generators of $\Lambda_{\Delta}^{2} M$ in low degree will be free-i.e., $\Lambda_{\Delta}^{2} M$ will have free summands generated in the lower degrees-but that there will definitely be degree one relations among the high degree minimal generators of $\Lambda_{\Delta}^{2} M$. In conclusion, we may see that $\Lambda_{\Delta}^{2} M$ is involutive and in general will be a mix of free modules generated in low degree together with involutive modules of resolution length one generated in all higher degrees.

4.5. The unmixed, overdetermined case. By definition this is an involutive exterior differential system whose Cartan characters are the same as for an overdetermined PDE system which is not a non-trivial iteration of several systems; it is expressed by the condition

$$
s_{1}=\cdots=s_{n-\ell}=s, \quad s_{n-\ell+1}=\cdots=s_{n}=0,
$$

which is equivalent to the tableau having the block form

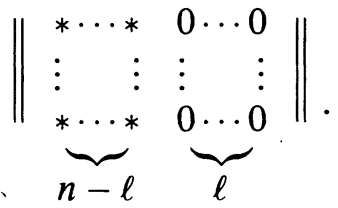

In this case we may solve for the last $\ell$ columns of the tableau matrix in terms of the first $n-\ell$, expressed by the relations

$$
\underline{\boldsymbol{\theta}}_{\lambda}=b_{\lambda}^{\rho} \underline{\boldsymbol{\theta}}_{\rho}, \quad 1 \leqq \rho, \sigma \leqq n-\ell, n-\ell+1 \leqq \lambda, \mu \leqq n .
$$


Written out fully these relations are

$$
\theta_{\lambda}^{\alpha}=b_{\lambda \beta}^{\rho \alpha} \theta_{\rho}^{\beta}
$$

Setting $B_{\lambda}(\xi)=b_{\lambda}^{\rho} \xi_{\rho}$, the involutivity of the symbol is equivalent to the commutation relations

$$
\left[B_{\lambda}(\xi), B_{\mu}(\xi)\right]=0 .
$$

The minimal free resolution of the graded $S$-module associated to the symbol is

$$
0 \rightarrow W_{\ell, .}^{*} \stackrel{\varphi_{\ell}}{\longrightarrow} \cdots \rightarrow W_{1, .}^{*} \stackrel{\varphi_{1}}{\longrightarrow} W^{*} \rightarrow M \rightarrow 0 .
$$

As will now be explained, this is essentially the Koszul complex constructed from the commuting "variables" $B_{\lambda}(\xi)$. For this we introduce a vector space $V$ of dimension $\ell$ and having a basis $v_{\lambda}$ where $n-\ell+1 \leqq \lambda \leqq n$. We set

$$
W_{k}^{*}=W^{*} \otimes_{\mathbb{F}} \Lambda^{k} V
$$

and define the mapping

$$
W_{1, .}^{*} \stackrel{\varphi_{1}}{\longrightarrow} W^{*}
$$

to be that induced by the assignment of generators

$$
\varphi_{1}\left(w^{\alpha} \otimes v_{\lambda}\right)=e_{\lambda} w^{\alpha}-b_{\lambda \beta}^{\rho \alpha} e_{\rho} w^{\beta}
$$

based on (2). Due to the commutation relations (3), this mapping induces

$$
W^{*} \otimes S V \rightarrow W^{*}
$$

and allows us to define a degree one mapping

$$
W^{*} \otimes \Lambda^{k} V \rightarrow W^{*} \otimes \Lambda^{k-1} V
$$

given by

$$
e_{I} w^{\alpha} \otimes v_{\lambda_{1}} \wedge \cdots \wedge v_{\lambda_{k}} \rightarrow \sum_{\nu}(-1)^{\nu-1} e_{I} \varphi_{1}\left(w^{\alpha} \otimes v_{\lambda_{\nu}}\right) v_{\lambda_{1}} \wedge \cdots \wedge \widehat{v_{\lambda_{\nu}}} \wedge \cdots \wedge v_{\lambda_{k}}
$$

where $\varphi_{1}$ is (4). This defines the above complex, which can be seen to be exact.

We will now show that

$$
H^{q}\left(\Lambda_{\Delta}^{p} M\right)=0, \quad 0 \leqq q<n-\ell .
$$

For this we retain the notations used in Sections 2.4 and 2.5, and we filter the complex $\Omega^{*}$ as follows: Set

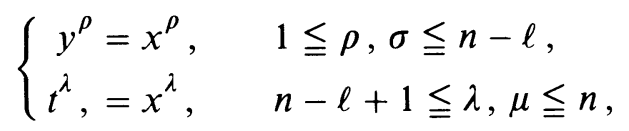

and write a general $\varphi \in \Omega^{*}$ as

$$
\varphi=\varphi_{\alpha_{1} \ldots \alpha_{p} R K}^{I_{1} \ldots I_{p}} \theta_{I_{1}}^{\alpha_{1}} \wedge \cdots \wedge \theta_{I_{p}}^{\alpha_{p}} \wedge d y^{R} \wedge d t^{K}, \quad\left|I_{\nu}\right| \subset\{1, \ldots, n-\ell\}
$$

and then define

$$
\overline{\bar{F}}^{a} \Omega^{*}=\{\varphi:|K| \geqq a\}
$$


That is, $\overline{\bar{F}}^{a} \Omega^{*}$ consists of forms that have at least $a$ differentials selected from the last $n-\ell$ coordinates. Clearly

$$
\begin{aligned}
\overline{\bar{F}}^{a+1} \Omega^{*} & \subset \overline{\bar{F}}^{a} \Omega^{*}, \\
d\left(\overline{\bar{F}}^{a} \Omega^{*}\right) & \subset \overline{\bar{F}}^{a} \Omega^{*}
\end{aligned}
$$

while the symbol relations (2) imply that

$$
\overline{\bar{F}}^{a} \Omega^{*} / \overline{\bar{F}}^{a+1} \Omega^{*} \cong \bigoplus \Lambda_{n-\ell}^{*-a} .
$$

More precisely, we identify $\Lambda_{n-\ell}^{*}$ with the complex of forms

$$
\psi=\psi_{\alpha_{1} \ldots \alpha_{p} R}^{I_{1} \ldots I_{p}} \theta_{I_{1}}^{\alpha_{1}} \wedge \cdots \wedge \theta_{I_{p}}^{\alpha_{p}} \wedge d y^{R}, \quad I_{\nu} \subset\{1, \ldots, n-\ell\} .
$$

Then for each multi-index $K$ with $|K|=a$, we have that $d t^{K} \wedge \psi \in \overline{\bar{F}}^{a} \Omega^{*}$ and this induces the isomorphism of vector spaces

$$
\overline{\bar{F}}^{a} \Omega^{*} / \overline{\bar{F}}^{a+1} \Omega^{*} \cong \bigoplus_{|K|=a} d t^{K} \wedge \Lambda_{n-\ell}^{*-a}
$$

inducing the isomorphism of complexes (6). The spectral sequence of the above filtered complex has

$$
\begin{aligned}
& \overline{\bar{E}}_{\infty}^{a, b} \Longrightarrow H^{a+b}\left(\Omega^{*}\right) \\
& \overline{\bar{E}}_{1}^{a, b}=0 \text { for } 0 \leqq b<n-\ell+a
\end{aligned}
$$

and this implies (5).

Remark. The proof shows that the vanishing result (5) does not require the involutivity of the symbol; it holds whenever the Cartan characters satisfy (1). This will be a completely general phenomenon-the inequality $\ell\left(\Lambda_{\Delta}^{p} M\right) \leqq \ell(M)$ does not require that the symbol be involutive. The much more subtle question of relating the minimal resolution of $\Lambda_{\Delta}^{p} M$ to that of $M$ will now be discussed in the first non-trivial overdetermined case $\ell=2$.

For this we write the relations (2) as

$$
\begin{aligned}
\theta_{n}^{\alpha} & =b_{\beta}^{\alpha \rho} \theta_{\rho}^{\beta}, \\
\theta_{n-1}^{\alpha} & =c_{\beta}^{\alpha \rho} \theta_{\rho}^{\beta} .
\end{aligned}
$$

The filtration introduced above has

$$
\begin{aligned}
& \bar{F}^{2}=\left\{\varphi_{\alpha_{1} \ldots \alpha_{p} K}^{I_{1} \ldots I_{p}} \theta_{I_{1}}^{\alpha_{1}} \wedge \cdots \wedge \theta_{I_{p}}^{\alpha_{p}} \wedge d y^{K} \wedge d t^{n-1} \wedge d t^{n}\right\}, \\
& \overline{\bar{F}}^{1}=\left\{\varphi_{\alpha_{1} \ldots \alpha_{p} K}^{\prime I_{1} \ldots I_{p}} \theta_{I_{1}}^{\alpha_{1}} \wedge \cdots \wedge \theta_{I_{p}}^{\alpha_{p}} \wedge d y^{K} \wedge d t^{n-1}\right. \\
& \left.+\varphi_{\alpha_{1} \ldots \alpha_{p} K}^{\prime I I_{1} \ldots I_{p}} \theta_{I_{1}}^{\alpha_{1}} \wedge \cdots \wedge \theta_{I_{p}}^{\alpha_{p}} \wedge d y^{K} \wedge d t^{n}\right\} \\
& \overline{\bar{F}}^{0}=\{\text { all forms } \varphi\} .
\end{aligned}
$$

Then we have

$$
\begin{cases}H^{q}\left(\bar{F}^{2}\right)=0, & q \neq n, \\ H^{q}\left(\bar{F}^{1} / \bar{F}^{2}\right)=0, & q \neq n-1, \\ H^{q}\left(\bar{F}^{0} / \bar{F}^{1}\right)=0, & q \neq n-2,\end{cases}
$$


and the $\bar{E}$ spectral sequence reduces to the commutative diagram

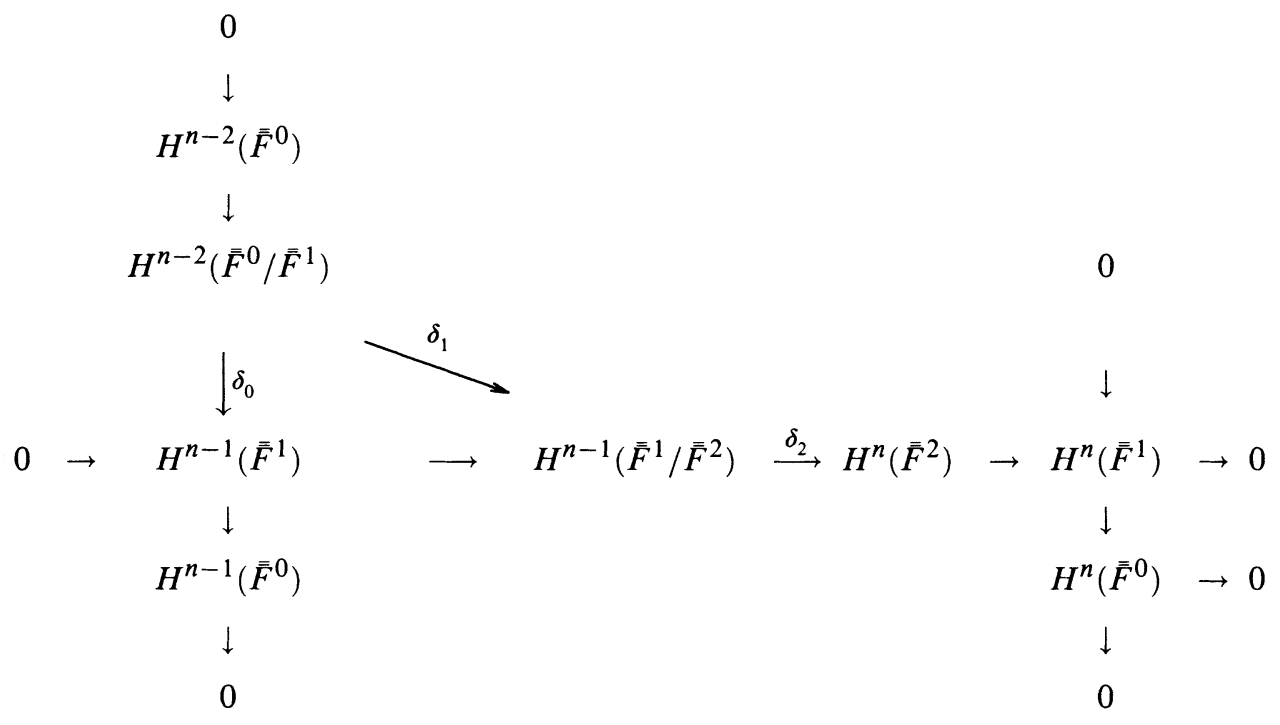

In the split case $\delta_{0}=\delta_{1}=\delta_{2}=0$. This has the interpretation that a resolution of $M$ lifts to one of $\Lambda_{\Delta}^{p} M$. Thus

$$
M \text { has }\left\{\begin{array}{l}
\text { generators } w^{\alpha}, \\
\text { generating relations } r_{n}: e_{n} w^{\alpha}=0, r_{n-1}: e_{n-1} w^{\alpha}=0, \\
\text { generating relation among the relations: } e_{n-1} r_{n}-e_{n} r_{n-1}=0
\end{array}\right.
$$

and when $p=2$

$$
\Lambda_{\Delta}^{2} M \text { has }\left\{\begin{array}{c}
\text { generators } \varphi=\varphi_{\alpha \beta}^{I} w^{\alpha} \wedge w^{\beta}, \varphi+(-1)^{|I| t} \varphi=0, \\
\text { generating relations } r_{n} \text { given by } e_{n} \varphi=0, \\
r_{n-1} \text { given by } e_{n-1} \varphi=0, \\
\text { generating relations among the relations } \\
\text { given by } e_{n-1} r_{n}-e_{n} r_{n-1}=0
\end{array}\right.
$$

corresponding to $H^{n}\left(\bar{F}^{2}\right), H^{n-1}\left(\bar{F}^{1} / \bar{F}^{2}\right), H^{n-2}\left(\bar{F}^{0} / \bar{F}^{1}\right)$ respectively in the above diagram.

In the non-split case the groups $H^{n}\left(\bar{F}^{2}\right), H^{n-1}\left(\bar{F}^{1} / \bar{F}^{2}\right), H^{n-2}\left(\overline{\bar{F}}^{0} / \bar{F}^{1}\right)$ are the same and represent what we may call "postulated" generators, relations, and relations among the relations. The actual generators are obtained by reducing the postulated generators modulo image $\delta_{2}$. The postulated relations are affected in two ways. First, they may not lift to actual relations-only those in kernel $\delta_{2}$ will do so. Secondly, those that do must be reduced by image $\delta_{1}$. Finally, the postulated relations among the relations $H^{n-2}\left(\bar{F}^{0} / \bar{F}^{1}\right)$ may not lift-only $\operatorname{ker} \delta_{1}=\operatorname{ker} \delta_{0}$ will do so. In all of this, however, we see that the minimal presentation of $\Lambda_{\Delta}^{2} M$, and indeed of a general $\Lambda_{\Delta}^{p} M$, has mappings of the same degree as in the split case. Thus $\Lambda_{\Delta}^{p} M$ is involutive. 
4.6. The mixed case. We shall now illustrate how the general case goes by considering a tableau of the form:

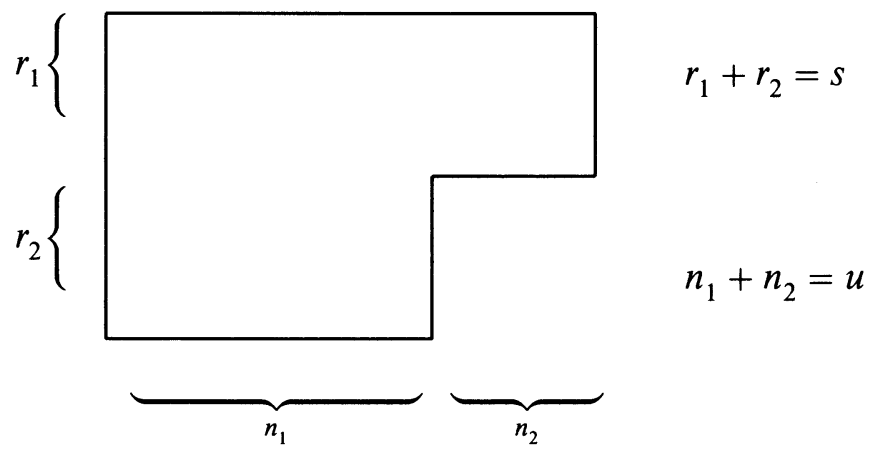

It is to be proved that

$$
H^{q}\left(\Lambda_{\Delta}^{p} M\right)=0, \quad 0 \leqq q<n_{1} .
$$

In the preceding section we separated the independent variables (horizontal axis) by setting

$$
\begin{aligned}
y^{\rho} & =x^{\rho}, & & 1 \leqq \rho, \sigma \leqq n_{1}, \\
t^{\lambda} & =x^{\lambda}, & & n_{1}+1 \leqq \lambda, \mu \leqq n,
\end{aligned}
$$

and filtering $\Omega^{*}$ by the images $\bar{F}^{a}$ of

$$
\Omega^{*} \otimes \Lambda^{a}\left\{d t^{n_{1}+1}, \ldots, d t^{n}\right\} \longrightarrow \Omega^{*+a} .
$$

In this case we shall also separate the dependent variables (vertical columns) as indicated by the blocks in the tableau and shall separate the effect of differentiation with respect to the two sets of dependent variables. This method will work in general, and, as in the previous sections, the vanishing result does not require involutivity of the tableau, this being needed only to insure the involutivity of the $\Lambda_{\Delta}^{p} M$. The main difficulty lies in organizing the notation.

Proceeding to the proof, we shall use the above ranges of indices and in addition shall let

$$
\left\{\begin{aligned}
& I, J, K \subset\left[1, \ldots, n_{1}\right], \\
& \quad R, S \subset\left[n_{1}+1, \ldots, n\right] .
\end{aligned}\right.
$$

The symbol relations are

$$
\theta_{I R}^{\alpha} \equiv 0 \bmod \left\{\theta_{J}^{\alpha}, \theta_{K S}^{\epsilon}\right\} \text { for }|R|>0, \alpha>r_{1},
$$

corresponding to the lower right-hand block $\alpha>r_{1}, i>n_{1}$ in the tableau. The forms $\theta_{I}^{\alpha}, \theta_{J R}^{\epsilon}$ are independent and we look at a typical spanning exterior monomial

$$
\theta=\theta_{I_{1}}^{\alpha_{1}} \wedge \cdots \wedge \theta_{I_{k}}^{\alpha_{k}} \wedge \theta_{J_{1} R_{1}}^{\epsilon_{1}} \wedge \cdots \wedge \theta_{J_{\ell} R_{\ell}}^{\epsilon_{\ell}} \wedge d y^{K} \wedge d t^{S}
$$

where all $\left|R_{\nu}\right|>0$. We set

$$
\begin{gathered}
\overline{\bar{w}}(\theta)=|S|, \\
\bar{F}^{a}=\{\text { forms } \varphi \text { with } \overline{\bar{w}}(\varphi) \geqq \varphi\},
\end{gathered}
$$


where this means that each monomial in the expression of $\varphi$ has at least $a$ $d t^{\lambda}$ 's. Then we have a $d$-stable filtration

$$
\bar{F}^{n_{2}} \subset \bar{F}^{n_{2}-1} \subset \cdots \subset \bar{F}^{1} \subset \bar{F}^{0}=\Omega^{*}
$$

and from

$$
\begin{aligned}
d \theta_{I}^{\alpha} & \equiv-\theta_{I \rho}^{\alpha} \wedge d y^{\rho} \bmod \left\{d t^{n_{1}+1}, \ldots, d t^{n}\right\}, \\
d \theta_{J R}^{\epsilon} & =-\theta_{J \rho R}^{\epsilon} \wedge d y^{\rho}-\theta_{J R \lambda}^{\epsilon} \wedge d t^{\lambda}
\end{aligned}
$$

we see that on the successive quotients $\overline{\bar{F}}^{a} / \bar{F}^{a+1}$ the exterior derivative only "sees" $d y^{1}, \ldots, d y^{n_{1}}$. More precisely, we let

$$
\begin{array}{r}
\overline{\bar{\Omega}}_{\underline{R}, S}^{k, \ell}=\{\text { span of the forms }(2) \text { where } S \text { with }|S|=a \text { and } \\
\left.\underline{R}=\left(R_{1}, \ldots, R_{\ell}\right) \text { is fixed }\right\} .
\end{array}
$$

Then

$$
\overline{\bar{F}}^{a} / \overline{\bar{F}}^{a+1} \cong \bigoplus_{\substack{k+\ell=p \\ \underline{R}, S}} \overline{\bar{\Omega}}_{\underline{R}, S}^{k, \ell} .
$$

If on $\bar{\Omega}_{\underline{R}, S}^{k, \ell}$ we set

$$
\begin{aligned}
& d^{\prime} \theta=d_{y}\left(\theta_{I_{1}}^{\alpha_{1}} \wedge \cdots \wedge \theta_{I_{k}}^{\alpha_{k}}\right) \wedge \theta_{J_{1} R_{1}}^{\epsilon_{1}} \wedge \cdots \wedge \theta_{J_{\ell} R_{\ell}}^{\epsilon_{\ell}} \wedge d y^{K} \wedge d t^{S} \\
& d^{\prime \prime} \theta=(-1)^{k} \theta_{I_{1}}^{\alpha_{1}} \wedge \cdots \wedge \theta_{I_{k}}^{\alpha_{k}} \wedge d_{y}\left(\theta_{J_{1} R_{1}}^{\epsilon_{1}} \wedge \cdots \wedge \theta_{J_{\ell} R_{\ell}}^{\epsilon_{\ell}}\right) \wedge d y^{K} \wedge d t^{S}
\end{aligned}
$$

where

$$
\begin{gathered}
d_{y} \theta_{I}^{\alpha}=-\theta_{I \rho}^{\alpha} \wedge d y^{\rho}, \\
d_{y} \theta_{J R}^{\epsilon}=-\theta_{J \rho R}^{\epsilon} \wedge d y^{\rho},
\end{gathered}
$$

then the induced differential $\overline{\bar{d}}$ on $\overline{\bar{F}}^{a} / \overline{\bar{F}}^{a+1}$ is

$$
\overline{\bar{d}}=d^{\prime}+d^{\prime \prime} \text {. }
$$

Moreover,

$$
\begin{gathered}
d^{\prime}: \overline{\bar{\Omega}}_{\underline{R}, S}^{k, \ell} \rightarrow \overline{\bar{\Omega}}_{\underline{R}, S}^{k+1, \ell}, \\
d^{\prime \prime}: \overline{\bar{\Omega}}_{\underline{R}}^{k, \ell} \rightarrow \overline{\bar{\Omega}}_{\underline{R}, \ell}^{k, \ell+1}, \\
d^{\prime 2}=d^{\prime \prime 2}=d^{\prime} d^{\prime \prime}+d^{\prime \prime} d^{\prime}=0 .
\end{gathered}
$$

The point is that both the $d^{\prime}$-cohomology and the $d^{\prime \prime}$-cohomology look like the unconstrained case, and consequently

$$
H^{q}\left(\overline{\bar{F}}^{a} / \overline{\bar{F}}^{a+1}\right)=0, \quad q<n_{1}+a .
$$

This implies that

$$
H^{q}\left(\Lambda_{\Delta}^{p} M\right)=0, \quad q<n_{1},
$$

as desired. 
The general case will be clear from a three-step tableau:

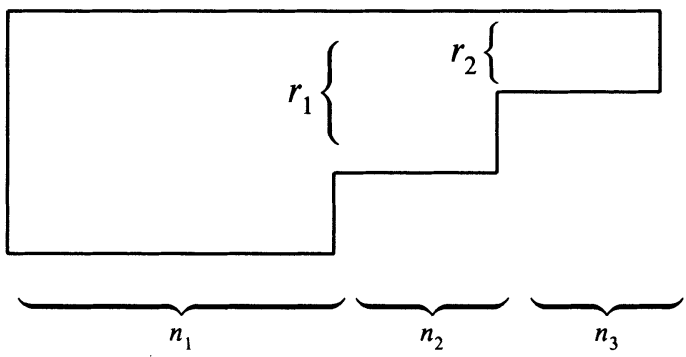

We keep the same splitting of independent variables (1) and filtration $\overline{\bar{F}}^{a}$ on $\Omega^{*}$ by the number of $d t^{\lambda}$ 's. We shall use the ranges of indices

$$
\left\{\begin{array}{l}
1 \leqq \epsilon \leqq r_{1}, 1 \leqq \eta \leqq r_{2} \\
1 \leqq i, j \leqq n_{1}, n_{1}+1 \leqq \rho, \sigma \leqq n_{1}+n_{2}, n_{1}+n_{2}+1 \leqq \lambda, \mu \leqq n
\end{array}\right.
$$

and corresponding blocks

$$
I=\left(i_{1}, \ldots, i_{k}\right), R, S=\left(\rho_{1}, \ldots, \rho_{\ell}\right), M=\left(\lambda_{1}, \ldots, \lambda_{m}\right) .
$$

The following are a basis for the 1 -forms in the ideal

$$
\theta_{I}^{\alpha}, \theta_{I R}^{\epsilon} \text { where }|R|>0, \quad \theta_{I R M}^{\eta} \text { where }|M|>0
$$

and forms in $\overline{\bar{F}}^{a}\left(F^{p} / F^{p+1}\right)$ are sums of monomials:

$$
\underbrace{\theta_{I_{1}}^{\alpha_{1}} \wedge \cdots \wedge \theta_{I_{k}}^{\alpha_{k}}} \wedge \underbrace{\theta_{I_{1} R_{1}}^{\epsilon_{1}} \wedge \wedge \theta_{I_{\ell} R_{\ell}}^{\epsilon_{\ell}}} \wedge \underbrace{\theta_{I_{1} S_{1} M_{1}}^{\eta_{1}} \wedge \wedge \theta_{I_{m} S_{m} M_{m}}^{\eta_{m}}} \wedge d y^{J} \wedge d t^{S}
$$

where $k+\ell+m=p,|S|+|J| \geqq a$. As above we may split the induced exterior differentiation on $\overline{\bar{F}}^{R} / \overline{\bar{F}}^{R+1}$ into

$$
\overline{\bar{d}}=d^{\prime}+d^{\prime \prime}+d^{\prime \prime \prime}
$$

where $d^{\prime}, d^{\prime \prime}, d^{\prime \prime \prime}$ are mutually graded-commuting and correspond to these vertical blocks in the above tableau. Each of $d^{\prime}, d^{\prime \prime}, d^{\prime \prime \prime}$ does not effect the index blocks $R_{1}, \ldots, R_{\ell}, S_{1} M_{1}, \ldots, S_{m} M_{m}$, and the individual cohomologies are all isomorphic to cohomology in the unconstrained case. Unwinding what this means yields the desired vanishing result.

\section{STRUCTURE OF THE CHARACTERISTIC COHOMOLOGY}

We have proved that $\bar{H}^{q}=0$ for $0<q<n-\ell$ where the characteristic number $\ell$ measures the degree of overdeterminedness of the exterior differential system. In this section we shall examine the structure of the remaining groups, focussing on the space $\mathscr{C}=\bar{H}^{n-\ell}$ of conservation laws for the system. This discussion will require that the exterior differential system be involutive, and it will show that $\mathscr{C}$ may be canonically identified with the kernel of a first-order linear differential operator between naturally defined vector bundles.

One may ask: Is this rather abstract theorem of practical value for computing conservation laws in specific examples? A fair question, one which we shall begin to address when we turn to the computation of examples later in this paper and, especially, in Part II. 
5.1. A variant of the Spencer complex. Let $(X, \mathscr{I})$ be the infinite prolongation of an involutive exterior differential system $\mathscr{J}_{0}$ on a manifold $X_{0}$. The exterior powers of $\mathscr{I}$ define a filtration $F^{p} \Omega^{*}(X)$ whose associated graded we have denoted by $\bar{\Omega}^{p, *}$ with induced differential $\bar{d}$. For $p \geqq 1$ there is then defined the (non-trivial) weight filtration $W_{k}\left(\bar{\Omega}^{p, *}\right)$ and we have denoted its associated graded by $\bar{\Omega}_{k}^{p, *}$ with induced differential $\delta$. The basic property is that $\delta$ is linear over the functions, and making suitable constant rank assumptions we denote by $\mathscr{H}_{k}^{p, q}$ the vector bundle over $X$ defined by the cohomology of the complex $\left\{\overline{\boldsymbol{\Omega}}_{k}^{p, *}, \delta\right\}$ :

We also set

$$
\mathscr{H}_{k}^{p, q}=H^{q}\left(\bar{\Omega}_{k}^{p, *}, \delta\right)
$$

$$
\mathscr{H}^{p, q}=\bigoplus_{k} \mathscr{H}_{k}^{p, q} .
$$

Now there is a spectral sequence $E_{r}^{p, q}$ abutting to $H^{*}(X)$ and with

$$
\begin{aligned}
& E_{1}^{p, q}=H^{q}\left(\bar{\Omega}^{p, *}, \bar{d}\right), \\
& E_{1}^{0, q}=\bar{H}^{q} \quad \text { (our primary object of study). }
\end{aligned}
$$

The weight filtration then gives a second spectral sequence $\left\{\bar{E}_{r}^{k, q}\right\}$ abutting to $E_{1}^{p, *}$ for each fixed $p>0$ and with

$$
\bar{E}_{1}^{k, q}=\mathscr{H}_{k}^{p, q}
$$

Our main result thus far has been to infer that $\bar{H}^{q}=0$ for $0<q<n-\ell$ by showing that (i) $\mathscr{H}^{p, q}=0$ for $p>0, q<n-\ell$, which implies (ii) $E_{1}^{p, q}=0$ for $p>0, q<n-\ell$, and then this implies the above stated vanishing result for the $\bar{H}^{q}$. We will now study some aspects of the structure of the nonzero part of the characteristic cohomology. To state the result, we denote by

$$
\nabla_{k}: \mathscr{H}_{k}^{p, q} \rightarrow \mathscr{H}_{k-1}^{p, q+1}, \quad p>0,
$$

the differential in the $\bar{E}_{r}^{k, q}$ spectral sequence, and we denote by

$$
\nabla: \mathscr{H}^{p, q} \rightarrow \mathscr{H}^{p, q+1}
$$

the direct sum over $k$ of the mappings (1). Then $\nabla^{2}=0$ and the cohomology of the complex $\left\{\mathscr{H}^{p, *}, \nabla\right\}$ computes the $E_{2}$-term of the weight spectral sequence for each $p>0$. Our result is given by the

Theorem. (i) In the involutive case

$$
E_{1}^{1, q}=H^{q}\left(\mathscr{H}^{1, *}, \nabla\right)
$$

(ii) For all $p \geqq 1$,

$$
E_{1}^{p, n-\ell} \subset \mathscr{H}^{p, n-\ell}
$$

is the subspace given by the kernel of a canonical linear differential operator.

Corollary. $\mathscr{C} \cong \operatorname{ker} \nabla \cap \operatorname{ker} d_{1}$ where $\nabla: \mathscr{H}^{1, n-\ell} \rightarrow \mathscr{H}^{1, n-\ell+1}$ and $d_{1}: E_{1}^{1, n-\ell} \rightarrow$ $E_{1}^{2, n-\ell}$ are canonically defined first-order linear differential operators. 
Proof. We have seen in Section 4.1 above (see equation (1) there) that $\mathscr{H}_{k}^{p, q}$ is the vector bundle whose typical fiber at a point $\xi$ is

$$
H_{k+q}^{q}\left(\Lambda_{\Delta}^{p} M_{\xi}\right)
$$

where $M_{\xi}$ is the graded module associated to the symbol of the exterior differential system at the point $\xi$, and where $H_{m}^{q}(\cdot)$ refers to (the dual of) Koszul homology in module degree $m$. The index $k+q$ appears above and the weight filtration encodes both the module degree and the exterior degree.

When $p=1$, since $M$ is involutive and is generated in degree zero, we have that

$$
H_{+}^{q}(M)=0 \text {. }
$$

Thus the weight spectral sequence degenerates at the $E_{2}$-term, and

$$
\bar{E}_{2}=H^{*}\left(\bar{E}_{1}, \nabla\right) \text {. }
$$

Unwinding the notation gives the first result.

The remaining part of the theorem follows from general properties of spectral sequences $\left\{\bar{E}_{r}^{k, q}\right\}$ with $\bar{E}_{1}^{k, q}=0$ for $0 \leqq q<n-\ell$. Q.E.D.

Discussion. The complex $\left\{\mathscr{H}^{1, *}, \nabla\right\}$ will be called the universal Spencer complex. We shall explain this point.

Referring to the notations introduced in Section 2.1 , in any local coordinate system $\left(x^{i}, u^{\alpha}, p_{i}^{\alpha}, p_{i j}^{\alpha}, \ldots\right)$ on $G_{n, \infty}\left(X_{0}\right)$ there is a splitting

$$
d=d_{V}+d_{H}
$$

of the exterior derivative into "vertical" and "horizontal" components. Explicitly,

$$
d_{V}=\sum_{|I|=0}^{\infty} \partial / \partial p_{K}^{\alpha} \theta_{K}^{\alpha}
$$

where we have set $p_{0}^{\alpha}=u^{\alpha}$,

$$
\left\{\begin{array}{l}
d_{H}=\sum D_{i} d x^{i} \\
D_{i}=\partial / \partial x^{i}+\sum_{|I|=0}^{\infty} \partial / \partial p_{I}^{\alpha} p_{I i}^{\alpha}
\end{array}\right.
$$

For any integral manifold

$$
f: N \rightarrow G_{n, \infty}\left(X_{0}\right)
$$

of the universal Pfaffian system $\theta_{I}^{\alpha}=0,|I| \geqq 0$, on $X$, we have

$$
\begin{aligned}
& f^{*} d_{V} \theta_{I}^{\alpha}=0, \\
& f^{*} d_{H} \theta_{I}^{\alpha}=0 .
\end{aligned}
$$

In fact, if the integral manifold is given by

$$
\left(x^{i}\right) \rightarrow\left(x^{i}, u^{\alpha}(x), p_{i}^{\alpha}(x), p_{i j}^{\alpha}(x), \ldots\right),
$$


then for any function $\varphi$ on $X$

$$
f^{*}\left(D_{i} \varphi\right)=\partial / \partial x^{i}\left(f^{*} \varphi\right)
$$

so that $D_{i}$ is a "universal form" of $\partial / \partial x^{i}$.

Let now $X \subset G_{n, \infty}\left(X_{0}\right)$ be the underlying manifold of infinite prolongation $\mathscr{I}$ of an involutive exterior differential system $\mathscr{J}_{0}$ on $X_{0}$. We have seen that the equations defining $X$ are

$$
D_{I} F=0
$$

where $F\left(x^{i}, u^{\alpha}, p_{i}^{\alpha}\right)$ varies over all the functions on $G_{*}\left(T X_{0}\right)$ defined by $\theta \in$ $\mathscr{I}_{0}^{n}$, i.e.,

$$
F(E)=\left.\theta\right|_{E}
$$

for $E \in G_{n}\left(T X_{0}\right)$. It follows that the vector fields $\left.D_{i}\right|_{X}$ are tangent to $X$ and induce vector fields on $X$ that we still denote by $D_{i}$. In these local coordinates the operators

$$
\nabla: \mathscr{H}^{1, q} \rightarrow \mathscr{H}^{1, q+1}
$$

are then easily seen to be horizontal, in the sense that

$$
\nabla=\sum A^{i} D_{i}+B
$$

where $A^{i}, B \in \operatorname{Hom}\left(\mathscr{H}^{1, q}, \mathscr{H}^{1, q+1}\right)$ are bundle maps that are linear over the functions. It follows that $\left\{\mathscr{H}^{1, *}, \nabla\right\}$ induces on any integral manifold

$$
f: N \rightarrow X_{0}
$$

of $\mathscr{J}_{0}$ a complex of vector bundles and first-order linear operators, which we shall denote by $\left\{\mathscr{H}_{f}^{*}, \nabla_{f}\right\}$. In fact, it may be seen that $\left\{\mathscr{H}_{f}^{*}, \nabla_{f}\right\}$ is just the dual of (a suitable relabelling of) the Spencer complex associated to the PDE system which is defined by the exterior differential system $\mathscr{I}_{0}$ in our particular local coordinate system (cf. Chapters IX, X of [2] for a discussion of the Spencer complex and further references).

One may invert this discussion. That is, not knowing what Spencer cohomology and the Spencer complex are, one is led naturally and inevitably to them once one agrees to study the characteristic cohomology of an exterior differential system.

The first term in the Spencer complex is

$$
\nabla_{f}: \mathscr{H}_{f}^{n-\ell} \rightarrow \mathscr{H}_{f}^{n-\ell+1}
$$

and it is an interesting point that the universal conservation laws appear naturally as a subspace of $\operatorname{ker} \nabla_{f}$ in (2).

What the theorem and its corollary give is that the space of conservation laws is given by the kernel of a canonical linear differential operator, modulo nothing. We have seen a special case of this in the contact and symplectic manifold examples in Section 1. In the case of determined PDE systems, this phenomena has been formulated in coordinates and used to compute examples by Vinogradov [15] and others. As we shall see in Part II of this paper, the result itself is of some use, but most importantly it serves to guide the generally complicated computations that arise in non-trivial applications of E. Cartan's equivalence method. 
5.2. Quadratic conservation laws for constant coefficient linear equations. As a first application of the general theory in the preceding section, we shall investigate the space of conservation laws associated to a constant coefficient linear PDE system. We first consider the determined case which in suitable coordinates may be written as

$$
\frac{\partial u^{\alpha}(x)}{\partial x^{n}}=b_{\beta}^{\alpha \rho} \frac{\partial u^{\beta}(x)}{\partial x^{\rho}}, \quad 1 \leqq \rho, \sigma \leqq n-1,
$$

where the $b^{\rho}=\left\|b_{\beta}^{\alpha \rho}\right\|$ are constant matrices. Associated to this system is its symbol and we denote by $M$ the graded module associated to the symbol. Denoting as usual by $\left(x^{i}, u^{\alpha}, p_{i}^{\alpha}, p_{i j}^{\alpha}, \ldots\right)$ the natural coordinates on the space $J^{\infty}\left(\mathbb{R}^{n}, \mathbb{R}^{s}\right)$ of infinite jets of mappings from $\mathbb{R}^{n}$ to $\mathbb{R}^{s}$, we observe that the group of gauge transformations that leave invariant the property that (1) be a linear, homogeneous constant coefficient equation will in general be equal to $G L_{n} \times G L_{s}$, and it is this symmetry group that we shall take. We are interested in conservation laws that are invariant under translation in $\mathbb{R}^{n}$, and for reasons to be explained below we shall focus on those represented in $E_{1}^{1, n-1}$ by translation invariant forms whose coefficients are linear in the jet coordinates $u^{\alpha}, p_{i}^{\alpha}, p_{i j}^{\alpha}, \ldots$. The following will also be explained below:

Definition. Conservation laws represented in $E_{1}^{1, n-1}$ by translation invariant forms whose coefficients are linear in the jet coordinates will be called quadratic conservation laws for the PDE system (1).

Theorem. The space of quadratic conservation laws for (1) is naturally isomorphic to $H^{n-1}\left(\operatorname{Sym}_{\Delta}^{2} M\right)$.

Thus, the $\Delta$-multilinear algebra appears once again, in a different form, in a natural way associated to a geometric problem.

Proof. We shall use the notation in the preceding section. For a point $\xi \in X$ we shall successively compute that part of

(i) $\mathscr{H}_{\xi}^{1, n-1}$,

(ii) $\nabla: \mathscr{H}^{1, n-1} \rightarrow \mathscr{H}^{1, n}$,

(iii) $d_{1}: \operatorname{ker} \nabla \rightarrow \mathscr{H}^{2, n-1}$

corresponding to forms that are translation invariant and linear in the jet coordinates. By the corollary in the preceding section, the kernel of (iii) is identified with the space of conservation laws for (1).

Step one. We first recall notation.

- $d x^{i}$ and $\theta_{I}^{i}$ are linear functions on two vector spaces, the $d x^{i}$ being linearly independent and the $\theta_{I}^{\alpha}$ subject to the relations

$$
b_{\alpha}^{\lambda i} \theta_{I i}^{\alpha}=0 .
$$

- $\Omega^{p, q}$ is spanned by the forms

$$
\theta_{I_{1}}^{\alpha_{1}} \wedge \cdots \wedge \theta_{I_{p}}^{\alpha_{p}} \wedge d x^{j_{1}} \wedge \cdots \wedge d x^{j_{q}}
$$

and $\Omega^{*}=\bigoplus \Omega^{p, q}$. 
- The differential

$$
d: \Omega^{p, q} \rightarrow \Omega^{p, q+1}
$$

is defined by $d\left(d x^{i}\right)=0$ and

$$
d \theta_{I}^{\alpha}=-\theta_{I i}^{\alpha} \wedge d x^{i}
$$

together with the usual properties of being a derivation of the exterior algebra.

- We will denote by $\Lambda_{m}^{*}=\bigoplus \Lambda_{m}^{p, q}$ a complex analogous to $\Omega^{p, q}$ but where the number of $d x^{i}$ 's is $m$ and where there are no relations on the $\theta_{I}^{\alpha}$-this "free" case models the unconstrained geometric situation in which the number of independent variables is $m$, and we have seen that

$$
H^{k}\left(\Lambda_{m}^{*}\right)=0, \quad k<m .
$$

In order to compute the fiber

$$
\mathscr{H}_{\xi}^{1, n-1} \cong H^{1, n-1}\left(\Omega^{*}, d\right)
$$

we proceed as in Section 4.4 and set

$$
\begin{aligned}
& \Omega^{p, q}=d x^{n} \wedge \Omega^{\prime p, q-1} \cong \Lambda_{n-1}^{p, q-1}[1], \\
& \Omega^{\prime p, q}=\Omega^{p, q} / \Omega^{p, q} \cong \Lambda_{n-1}^{p, q} .
\end{aligned}
$$

In the exact cohomology sequence

$$
0 \rightarrow H^{1, n-1}\left(\Omega^{*}\right) \rightarrow H^{1, n-1}\left(\Omega^{\prime * *}\right) \stackrel{\delta}{\longrightarrow} H^{1, n}\left(\Omega^{\prime *}\right)
$$

we have $\boldsymbol{\delta}=0$ as noted in Section 4.4. In fact, given

$$
\varphi^{\prime \prime}=f_{\alpha} \theta^{\alpha} \wedge d x_{n} \in H^{1, n-1}\left(\Omega^{\prime \prime *}\right)
$$

we see that

$$
\varphi=f_{\alpha} \theta^{\alpha} \wedge d x_{n}-f_{\beta} b_{\alpha}^{\beta \rho} \theta^{\alpha} \wedge d x_{\rho}
$$

satisfies

$$
\delta \varphi=0
$$

and thus gives a class $\varphi \in H^{1, n-1}\left(\Omega^{*}\right)$ which serves to provide a lifting of the class defined by $\varphi^{\prime \prime} \in H^{1, n-1}\left(\Omega^{\prime \prime *}\right)$.

Step two. We will consider sections

$$
\varphi=f_{\alpha} \theta^{\alpha} \wedge d x_{n}-f_{\beta} b_{\alpha}^{\beta \rho} \theta^{\alpha} \wedge d x_{\rho}
$$

of $\mathscr{U}^{1, n-1}$ where $f_{\alpha}$ is a function on $X$. In fact, we shall consider functions of the form

$$
f_{\alpha}=\sum_{|I| \geq 0, \beta} f_{\alpha \beta}^{I} p_{I}^{\beta}
$$


that are linear in the fiber coordinates, and where we set $u^{\alpha}=p_{0}^{\alpha}$. As noted above, this has intrinsic meaning, and by a scaling argument in the independent variables $x^{i}$ we see that it will suffice to consider the case where

$$
f_{\alpha}=\sum_{|I|=k, \beta} f_{\alpha \beta}^{I} p_{I}^{\beta}
$$

is homogeneous of degree $-k$ in the dependent variables. We want to compute

$$
\nabla: \mathscr{H}^{1, n-1} \rightarrow \mathscr{H}^{1, n}
$$

for $\varphi \in \mathscr{H}^{1, n-1}$ of this form. By definition

$$
\begin{aligned}
\nabla \varphi & =d \varphi \bmod \mathscr{I}_{\wedge \mathcal{I}} \\
& =\left(D_{n} f_{\alpha}-D_{\rho} f_{\beta} b_{\alpha}^{\beta \rho}\right) \theta^{\alpha} \wedge d x \\
& =\left(f_{\alpha \beta}^{I} p_{I n}^{\beta}-f_{\beta \gamma}^{I} p_{I \rho}^{\gamma} b_{\alpha}^{\beta \rho}\right) \theta^{\alpha} \wedge d x \\
& =\left(f_{\alpha \beta}^{I} b_{\gamma}^{\beta \rho}-b_{\alpha}^{\beta \rho} f_{\beta \gamma}^{I}\right) p_{I \rho}^{\gamma} \theta^{\alpha} \wedge d x .
\end{aligned}
$$

If we set

$$
\begin{aligned}
& \varphi(\xi)=\left\|f_{\alpha \beta}(\xi)\right\|=\left\|f_{\alpha \beta}^{I} \xi_{I}\right\|, \\
& b(\xi)=\left\|b_{\alpha}^{\beta \rho} \xi_{\rho}\right\|,
\end{aligned}
$$

then we have

$$
\nabla \varphi=0 \Longleftrightarrow[\varphi(\xi), b(\xi)]=0 .
$$

We note the analogy with the proposition in Section 4.4, where the condition $[\varphi(\xi), b(\xi)]=0$ arose in a different context. We note there the symmetry condition

$$
\varphi+(-1)^{|I| t} \varphi=0
$$

that was imposed on $\varphi$.

Step three. We assume that

$$
\varphi=f_{\alpha \beta}^{I} p_{I}^{\beta} \theta^{\alpha} \wedge d x_{n}-f_{\beta \gamma}^{I} p_{I}^{\gamma} b_{\alpha}^{\beta \rho} \theta^{\alpha} \wedge d x_{\rho} \in E_{1}^{1, n-1}
$$

satisfies $\nabla \varphi=0$, and we want to compute the condition

$$
d_{1} \varphi=0 \text { in } E_{1}^{2, n-1} \text {. }
$$

For this we denote by $E_{1}^{\prime}, E_{1}^{\prime \prime}$ the spectral sequences associated to the bigraded complexes $\Omega^{\prime *}, \Omega^{\prime \prime *}$ respectively. Then we have a diagram

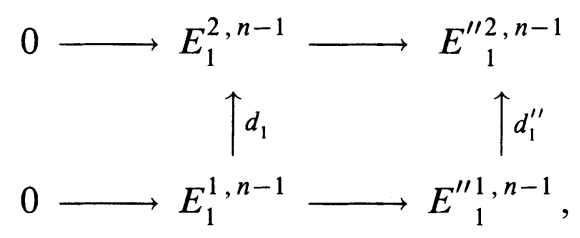

from which we see that

$$
d_{1} \varphi=0 \Longleftrightarrow d_{1}^{\prime \prime} \varphi^{\prime \prime}=0
$$


where

$$
\varphi^{\prime \prime}=f_{\alpha \beta}^{I} p_{I}^{\beta} \theta^{\alpha} \wedge d x_{n}
$$

is the image in $E_{1}^{11^{1, n-1}}$ of $\varphi \in E_{1}^{1, n-1}$.

Now

$$
d_{1}^{\prime \prime} \varphi^{\prime \prime}=f_{\alpha \beta}^{I} \theta_{I}^{\beta} \wedge \theta^{\alpha} \wedge d x_{n} .
$$

Setting $f_{\alpha \beta}^{I}=\hat{f}_{\alpha \beta}^{I}+\hat{\hat{f}}_{\alpha \beta}^{I}$ where

$$
\begin{aligned}
& \hat{f}_{\alpha \beta}^{I}=\frac{1}{2}\left(f_{\alpha \beta}^{I}+(-1)^{|I|} f_{\beta \alpha}^{I}\right), \\
& \hat{\hat{f}}_{\alpha \beta}^{I}=\frac{1}{2}\left(f_{\alpha \beta}^{I}-(-1)^{|I|} f_{\beta \alpha}^{I}\right),
\end{aligned}
$$

we see from equation (3) in Section 3.2 that

$$
d_{1}^{\prime \prime} \varphi^{\prime \prime}=0 \Longleftrightarrow \hat{f}_{\alpha \beta}^{I}=0 \text {. }
$$

Thus

$$
\left\{\nabla \varphi=d_{1} \varphi=0\right\} \Leftrightarrow\left\{\begin{array}{l}
\varphi=f_{\alpha \beta}^{I} p_{I}^{\beta} \theta^{\alpha} \wedge d x_{n}-f_{\beta \gamma}^{I} p_{I}^{\alpha} b_{\alpha}^{\beta \rho} \theta^{\alpha} \wedge d x_{\rho} \\
\text { where }[\varphi(\xi), b \xi)]=0 \text { and } \\
\varphi(\xi)-(-1)^{|I| t} \varphi(\xi)=0
\end{array}\right\} .
$$

By (3) in Section 3.2 the space of $\varphi$ satisfying the condition

$$
\varphi(\xi)-(-1)^{|I| t} \varphi(\xi)=0
$$

is isomorphic to $H^{n-1}\left(\operatorname{Sym}_{\Delta}^{2} M^{\prime}\right)$ where $M^{\prime}$ is the free module over $S^{\prime}=$ $\mathbb{F}\left[e_{1}, \ldots, e_{n-1}\right]$ with generators $w^{1}, \ldots, w^{s}$. It remains to identify those $\varphi$ satisfying both conditions on the right-hand side of (3) with $H^{n-1}\left(\operatorname{Sym}_{\Delta}^{2} M\right)$. This is done in essentially the same way as for $\Lambda_{\Delta}^{2} M^{\prime}$, and we will only sketch the argument.

In general, to compute the $H^{q}\left(\operatorname{Sym}_{\Delta}^{p} M\right)$ we consider the complex $\widetilde{\Omega}^{*}=$ $\bigoplus \widetilde{\Omega}^{p, q}$ of forms

$$
\psi=\psi_{\alpha_{1} \ldots \alpha_{\rho} J}^{I_{1} \ldots I_{p}} \theta_{I_{1}}^{\alpha_{1}} \circ \cdots \circ \theta_{I_{p}}^{\alpha_{p}} \otimes d x^{J}, \quad|J|=q,
$$

where the $\theta_{I_{\nu}}^{\alpha_{\nu}}$ are independent forms multiplied symmetrically. We define a differential $\delta$ by linearity and

$$
\begin{gathered}
\delta\left(\theta_{I_{1}}^{\alpha_{1}} \circ \cdots \circ \theta_{I_{p}}^{\alpha_{p}} d x^{J}\right)=\sum_{\nu} \theta_{I_{1}}^{\alpha_{1}} \circ \cdots \circ\left(\delta \theta_{I_{\nu}}^{\alpha_{\nu}}\right) \circ \cdots \circ \theta_{I_{p}}^{\alpha_{p}} \otimes d x^{J}, \\
\delta\left(\theta_{I}^{\alpha}\right)=\theta_{I \rho}^{\alpha} \otimes d x^{\rho}+b_{\beta}^{\alpha \rho} \theta_{I \rho}^{\beta} \otimes d x^{n}, \quad 1 \leq \rho \leq n-1,
\end{gathered}
$$

where we move multiplication by $d x^{j}$ across the $\theta_{I_{\mu}}^{\alpha_{\mu}}$ and exteriorly multiply it with $d x^{J}$. Thus, more or less by definition, the cohomology of this complex at the place with $p \quad \theta$ 's and $q d x^{j}$ 's computes $H^{q}\left(\operatorname{Sym}_{\Delta}^{p} M\right)$. 
We next set $\widetilde{\mathbf{\Omega}}^{\prime *}=d x^{n} \wedge \widetilde{\mathbf{\Omega}}^{*}$ and $\widetilde{\mathbf{\Omega}}^{\prime \prime *}=\widetilde{\mathbf{\Omega}}^{*} / \widetilde{\mathbf{\Omega}}^{\prime *}$, and note as in the case of $\Lambda_{\Delta}^{p} M$ we have

From the exact cohomology sequence

$$
\begin{cases}H^{q}\left(\widetilde{\boldsymbol{\Omega}}^{\prime *}\right)=0, & q<n, \\ H^{q}\left(\widetilde{\boldsymbol{\Omega}}^{\prime \prime *}\right)=0, & q<n-1 .\end{cases}
$$

$$
0 \rightarrow H^{n-1}\left(\widetilde{\mathbf{\Omega}}^{2, *}\right) \rightarrow H^{n-1}\left(\widetilde{\mathbf{\Omega}}^{\prime 2, *}\right) \stackrel{\tilde{\delta}}{\longrightarrow} H^{n}\left(\widetilde{\mathbf{\Omega}}^{\prime 2, *}\right)
$$

for a class in $H^{n-1}\left(\widetilde{\Omega}^{\prime \prime 2, *}\right)=H^{n-1}\left(\operatorname{Sym}_{\Delta}^{2} M^{\prime}\right)$ represented by

$$
\tilde{\varphi}^{\prime \prime}=f_{\alpha \beta}^{I} \theta_{I}^{\alpha} \circ \theta^{\beta} \otimes d x_{n}, f_{\alpha \beta}^{I}-(-1)^{|I|} f_{\beta \alpha}^{I}=0
$$

we have as in the proposition in Section 4.4 that

$$
\delta \tilde{\varphi}^{\prime \prime}=0 \Longleftrightarrow[\varphi(\xi), b(\xi)]=0 .
$$

Since $\operatorname{ker} \delta \cong H^{n-1}\left(\operatorname{Sym}_{\Delta}^{2} M\right)$, we have completed the proof of the theorem.

Discussion. The conservation laws $\mathscr{C}=E_{1}^{0, n-1}$ map injectively to $E_{1}^{1, n-1}$ by

$$
d_{1}: E_{1}^{0, n-1} \rightarrow E_{1}^{1, n-1} \text {. }
$$

The above theorem identifies the classes in $E_{1}^{1, n-1}$ that are in the image of $d_{1}$ and that are linear in the jet coordinates. But since $d_{1}$ is determined by the rules

$$
\begin{aligned}
& d_{1}\left(\theta_{I}^{\alpha}\right)=d_{1}\left(d x^{j}\right)=0, \\
& d_{1}(f)=\sum \partial f / \partial p_{I}^{\alpha} \theta_{I}^{\alpha},
\end{aligned}
$$

we see that $d_{1}$ maps exactly those classes in $E_{1}^{0, n-1}$ that are quadratic in the $p_{I}^{\alpha}$ 's to classes in $E_{1}^{1, n-1}$ that are linear in these coordinates. This explains the above definition.

Now, in the classical calculus of variations perhaps the most interesting Lagrangians are those of the form

$$
L\left(x^{i}, u^{\alpha}, p_{i}^{\alpha}, \ldots, p_{I}^{\alpha}\right) d x, \quad|I| \leqq k,
$$

which are quadratic in the highest order terms. Similarly, as illustrated by the various classical conservation laws and the $\mathrm{KdV}$, we may expect conservation laws that are quadratic in their highest order terms to be of particular interest. It is for this reason that we have focussed on the quadratic conservation laws in the above theorem. We note that finding these indirectly by studying classes in $E_{1}^{1, n-1}$ that are linear in the $p_{I}^{\alpha}$ and satisfy certain conditions appears to be considerably more efficient than directly studying the quadratic conservation laws.

\section{Global Results}

The results obtained thus far are microlocal, in a precise sense to be explained below. In this final section we shall give a global theorem in cohomology, one which follows in a more or less standard way from our local result, and then we shall discuss a more geometric dual set of questions which pertain to a very prescient theorem of Thom [10]. 
6.1. A relation between ordinary and characteristic cohomology. Let $\mathscr{I}_{0}$ be an involutive exterior differential system on a connected manifold $X_{0}$, and as above denote by $\left(X_{k}, \mathscr{I}_{k}\right)$ the $k$-th prolongation with $X=\lim X_{k}$ and $\mathscr{I}=$ $\mathscr{J}_{0} \cup_{k>0} \mathscr{I}_{k}$ (cf. Section 1.3). Since $\mathscr{I}_{0}$ may not be a quasi-linear Pfaffian differential system, we need to be a little careful about what is meant that $\mathscr{I}_{0}$ be involutive. Denote by $G_{n}\left(\mathscr{J}_{0}\right) \subset G_{n}\left(T X_{0}\right)$ the set of $n$-dimensional integral elements of $\mathscr{J}_{0}$. A priori, $G_{n}\left(\mathscr{I}_{0}\right)$ is locally the locus of common zeros of a collection of smooth functions defined in an open set of $G_{n}\left(T X_{0}\right)$ (these functions are of the special form given in Section 1.3, but that only serves to restrict somewhat the singularities of $G_{n}\left(\mathscr{J}_{0}\right)$ in the fibers of $\left.G_{n}\left(T X_{0}\right) \rightarrow X_{0}\right)$. We shall make the following regularity assumptions:

There exists a connected, locally closed submanifold $X_{1}$ of $G_{n}\left(\mathscr{J}_{0}\right)$ such that

(i) each point $E \in X_{1}$ has a neighborhood $U$ in $G_{n}\left(T X_{0}\right)$ such that $U \cap$ $X_{1}=U \cap G_{n}\left(\mathscr{J}_{0}\right)$ and such that the forms in $\mathscr{J}_{0}$ give a regular set of defining equations for $U \cap X_{1}$,

(ii) $X_{1}$ consists of ordinary integral elements, and

(iii) the projection $X_{1} \rightarrow X_{0}$ is a submersion.

The concept that $E \in G_{n}(\mathscr{F})$ be ordinary is also explained in Chapter III of [2]; in the real analytic case it implies that there is a local integral manifold of $\mathscr{F}_{0}$ that is tangent to $E$. It is also shown there that with the assumptions (i)-(iii) the prolongation tower $\left(X_{k}, \mathscr{J}_{k}\right)$ exists and satisfies the conditions of Section 1.3 above. In particular, for $k \geqq 1$ the submersions $X_{k+1} \rightarrow X_{k}$ are fibrations whose fibers are affine linear spaces, and thus the projections $X_{k} \rightarrow X_{1}$ are all homotopy equivalences. (For more detail, the reader may want to consult Chapter VI of [2] as well.)

By microlocal we shall mean in a sufficiently small neighborhood in $X_{1}$ of an ordinary integral element $E \in X_{1}$. Our main vanishing result, given by Theorem 2 in Section 4.2, states that in the appropriate range the characteristic cohomology vanishes microlocally, i.e., it vanishes in the range $0<q<n-\ell$ in open sets $\pi_{1}^{-1}(U) \subset X$ where $\pi_{1}: X \rightarrow X_{1}$ is the projection and $U \subset X_{1}$ is a sufficiently small open set. We shall now prove

Theorem 1. Let $\mathscr{I}_{0}$ be an involutive exterior differential system of character $\ell$ and with trivial infinite derived system. ${ }^{2}$ Then we have

(i) $H^{q}(X, \mathbb{R}) \cong \bar{H}^{q}(X), 0 \leqq q<n-\ell$,

(ii) $0 \rightarrow H^{n-\ell}(X, \mathbb{R}) \rightarrow \bar{H}^{n-\ell}(X) \rightarrow H^{0}\left(\mathscr{\mathscr { \ell }}^{n-\ell}\right) \rightarrow H^{n-\ell+1}(X, \mathbb{R}) \rightarrow 0$

where $\overline{\mathscr{H}}^{q}$ is the q-th characteristic cohomology sheaf on $X$.

Proof. We recall that the topology on $X$ is defined by taking the open sets to be $\pi_{k}^{-1}(U)$ where $U \subset X_{k}$ is an open set and $\pi_{k}: X \rightarrow X_{k}$ is the projection. Then we define the sheaves $\underline{\Omega}^{q}, \underline{\mathscr{I}}^{q}, \underline{\boldsymbol{\Omega}}^{q}=\underline{\Omega}^{q} / \underline{\mathscr{I}}^{q}$, by considering the appropriate

\footnotetext{
${ }^{1}$ In Chapter III of [2] there is an explanation of how forms on $X_{0}$ give functions on $G_{n}\left(T X_{0}\right)$ and what it means for a set of such functions to give a regular set of defining equations.

${ }^{2}$ This means that there are locally no non-constant functions $f$ with $d f \in \mathscr{J}_{0}$-geometrically, it means that locally there are no "inessential variables" in the system.
} 
smooth forms in the open sets $\pi_{k}^{-1}(U)$. We then have a complex of sheaves over $X$

$$
\underline{\overline{\mathbf{\Omega}}}=\left\{\underline{\overline{\mathbf{\Omega}}}^{0} \stackrel{\bar{d}}{\longrightarrow} \underline{\overline{\mathbf{\Omega}}}^{1} \stackrel{d}{\longrightarrow} \cdots \stackrel{\bar{d}}{\longrightarrow} \underline{\boldsymbol{\Omega}}^{q} \stackrel{\bar{d}}{\longrightarrow} \underline{\mathbf{\Omega}}^{q+1} \stackrel{\bar{d}}{\longrightarrow} \cdots\right\},
$$

and we denote by $\overline{\mathscr{H}}^{q}$ the cohomology sheaves. By the assumption that $\mathscr{F}_{0}$ has a trivial infinite derived system, it follows that

$$
\overline{\mathscr{H}}^{0} \cong \mathbb{R}
$$

is the constant sheaf. Our microlocal vanishing theorem gives

$$
\overline{\mathscr{H}}^{q}=0 \quad \text { for } \quad 0<q<n-\ell .
$$

The results (i), (ii) are then a standard consequence of these two facts.

In more detail, denote by $\mathbb{H}\left(\underline{\bar{\Omega}}^{*}\right)$ the hypercohomology of the complex of sheaves $\overline{\underline{\Omega}}$. Then there are two spectral sequences ' $E$ and " $E$ each abutting to $\mathbb{H}\left(\underline{\bar{\Omega}}^{\cdot}\right)$, one where

$$
{ }^{'} E^{p, q}=H_{\delta}^{p}\left(\overline{\mathscr{H}}^{q}\right)
$$

and the other where

$$
" E_{1}^{p, q}=H_{d}^{q}\left(H_{\delta}^{p}(\underline{\overline{\boldsymbol{\Omega}}})\right) .
$$

Here, $\delta$ is used to denote the Čech cohomology of the sheaf in question. But, by the usual partition of unity argument, $H_{\delta}^{p}(\underline{\underline{\Omega}})=0$ for $p>0$ and so

$$
" E_{1}^{p, q}= \begin{cases}0, & p>0 \\ \bar{H}^{q}(X), & p=0 .\end{cases}
$$

On the other hand, ' $E_{1}^{p, q}=0$ for $0<q<n-\ell$, and the "picture" of the ' $E_{1}$ is

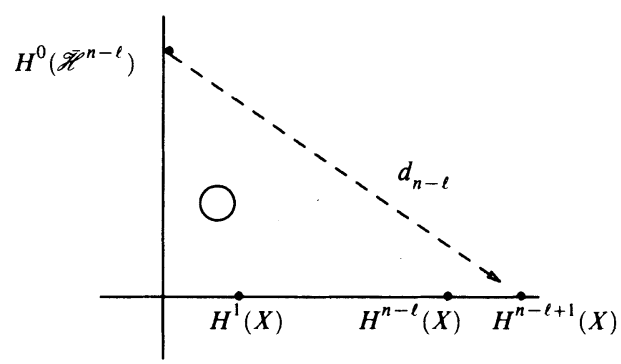

The result follows from this. Q.E.D.

6.2. $\mathcal{I}$-homology. Let $\mathscr{J}$ be an exterior differential system on a manifold $Y$. We let $\Delta^{q}$ denote a standard $q$-simplex and consider mappings

$$
f: \Delta^{q} \longrightarrow Y
$$

that are of class $C^{1}$ on $\Delta^{q}$, including on the boundary, and that satisfy

$$
f^{*} \theta=0, \quad \theta \in \mathcal{F} .
$$


We will call such a simplex a $\mathscr{J}$-simplex, and we may then define a $\mathscr{J}$-chain to be a singular chain each of whose simplices is a $\mathscr{J}$-simplex. The boundary of a $\mathscr{J}$-chain is again a $\mathscr{J}$-chain, and we denote by

$$
H_{*, \mathscr{J}}(Y)
$$

the resulting homology.

Definition. We shall call $H_{*, \mathscr{J}}(Y)$ the $\mathscr{J}$-homology of the exterior differential system $\mathscr{J}$.

This concept has been introduced by Thom [10] in the special case when $Y$ is an open set of $J^{k}\left(\mathbb{R}^{n}, \mathbb{R}^{s}\right)$ and $\mathscr{J}$ is the restriction to $Y$ of the contact system. He proved a result which is in a certain sense dual to the 1-line theorem of Vinogradov (i.e., Vinogradov's result [13] in the unconstrained case), although it neither implies nor is implied by Vinogradov's theorem. We will formulate and discuss a possible extension of Thom's result. It must be emphasized that the following discussion is highly speculative and is not intended to be anything more than suggestive. Apparently, there are great technical difficulties to be overcome before an actual proof of any of these results can be constructed. We have included this discussion here mainly because it indicates a geometric interpretation of the characteristic cohomology which we believe will have great importance for the theory.

First, remark that if we now denote the characteristic cohomology of the complex $\left(\Omega^{*}(Y) / \mathscr{J}, \bar{d}\right)$ by

$$
H_{\mathscr{J}}^{*}(Y)=H^{*}\left(\Omega^{*}(Y) / \mathcal{J}, \bar{d}\right)
$$

then there is a natural pairing

$$
H_{\mathscr{J}}^{q}(Y) \otimes H_{q, \mathscr{J}}(Y) \rightarrow \mathbb{R} .
$$

A natural question then is whether the $\mathscr{J}$-de-Rham Theorem holds. In other words, is the pairing (1) non-degenerate? We will see that, although in general the answer appears to be no, the question presents some very interesting and highly non-trivial issues.

There is always a natural mapping

$$
H_{q, \mathscr{J}}(Y) \rightarrow H_{q}(Y)
$$

(homology with $\mathbb{Z}$-coefficients), and by analogy with Theorem 1 in Section 6.1 above, we may pose the

Question. In case $\mathscr{F}_{0}$ is an involutive exterior differential system of character $\ell$ with trivial infinite derived system, do we have

$$
\begin{cases}\text { (i) } H_{q, \mathscr{J}}(X) \stackrel{\sim}{\rightarrow} H_{q}(X), & 0 \leqq q<n-\ell, \\ \text { (ii) } H_{n-\ell, \mathscr{J}}(X) \rightarrow H_{n-\ell}(X) \rightarrow 0 ? & \end{cases}
$$

Thom's result is that the answer to the same question for $Y$ an open subset of $J^{k}\left(\mathbb{R}^{n}, \mathbb{R}^{s}\right)$ is affirmative, so that we have

$$
\begin{aligned}
& H_{q, \mathscr{J}}(Y) \stackrel{\simeq}{\rightarrow} H_{q}(Y), \quad 0 \leqq q<n, \\
& H_{n, \mathscr{J}}(Y) \rightarrow H_{n}(Y) \rightarrow 0 .
\end{aligned}
$$


We will indicate how the type of arguments Thom used might be used to establish (i) and (ii) above in the case where $\mathscr{F}_{0}$ satisfies the condition that it "has no real Cartan characteristics in the range $0 \leqq q<n-\ell$ ", a condition that we shall explain below and which is satisfied for the contact systems.

A technical issue that arises concerns the level of smoothness that we require of $\mathscr{J}$-chains. Thus consider the induced mappings

$$
H_{q, \mathscr{I}_{k+1}}\left(X_{k+1}\right) \rightarrow H_{q, \mathscr{I}_{k}}\left(X_{k}\right), \quad k \geqq 1 .
$$

These mappings are all isomorphisms in ordinary homology and $H_{*}(X) \cong$ $H_{*}\left(X_{k}\right)$ for all $k \geqq 1$. If we have a piecewise- $C^{j} \mathscr{J}_{k}$-chain $\Gamma_{k}$ on $X_{k}$, then its prolongation $\Gamma_{k}^{*}$ is a piecewise $C^{j-1}$ chain on $X_{k+1}$. But unless $j \geqq 2$ we cannot say that $\Gamma_{k}^{*}$ will be a $\mathscr{I}_{k+1}$-chain. Moreover, even if $j \geqq 2$ and $\Gamma_{k}$ is an $\mathscr{I}_{k}$-cycle on $X_{k}$, it may not be the case that $\Gamma_{k}^{*}$ is an $\mathscr{J}_{k+1}$-cycle since the edges of $\Gamma_{k}$ may separate under prolongation. Although we see no real reason why (3) should fail to be isomorphisms for all $q$, we shall not pursue this matter here and shall restrict our attention to $X_{1}$. Similar arguments to those below will apply to the other $X_{k}$.

Next, we point out that the question (2) is essentially local. That is, suppose that for sufficiently small neighborhoods $U$ of each point in $X_{1}$ we have for the reduced $\mathscr{I}_{1}$-homology groups

$$
\tilde{H}_{q, \mathscr{F}_{1}}(U)=0, \quad 0 \leqq q<n-\ell .
$$

Then by standard local-to-global arguments such as those used in the preceding section, we may infer (2) with $X_{1}$ replacing $X$.

To discuss (4) it will be notationally more convenient to use cubes, denoted by $\square^{q}$ or simply $\square$ if the dimension is understood, rather than simplices. To establish (4) the following sort of result must be proved:

Let $0 \leqq q<n-\ell$ and let $\Sigma \subset \partial \square^{q+1}$ be a set of $q$-dimensional faces of the boundary of a $(q+1)$-cube. Suppose that

$$
f: \Sigma \rightarrow U
$$

is an $\mathscr{J}_{1}$-integral manifold that has an extension as a piecewise$C^{1}$ mapping to all of $\square^{q+1}$. Then $f$ may be extended to a piecewise- $C^{1} \mathscr{I}_{1}$-integral manifold. ${ }^{3}$

By arguments of the type used by Thom, the crucial case is when $\Sigma=\partial \square^{q+1}$ and this is the situation that we shall discuss.

Thus, let $0 \leqq q<n-\ell$ and let

$$
f: \partial \square^{q+1} \rightarrow U
$$

be a piecewise- $C^{1} \mathscr{I}_{1}$-integral manifold where $U$ is a sufficiently small neighborhood of a point in $X_{1}$. We shall sketch arguments to the effect that:

(i) In case (6) is in general position, $f$ may be extended to a piecewise- $C^{1}$ $\mathscr{I}_{1}$-integral manifold.

${ }^{3}$ This means that $\square$ may be subdivided and a continuous mapping $f$ exists which is a $C^{1}$ integral manifold on each of the subcubes. 
(ii) In case there are no real Cartan characteristics, we may deform $f$ to be in general position.

The essential case to understand (i) is when $s_{0}=s_{1}=s_{2}>s_{3} \geqq 0$. We shall take first the case $s_{3}=0$ (and $n=3$ ), and then indicate the modifications necessary in general.

Locally we may choose coordinates $(x, y, z, u, p, q, r)$ in $J^{1}\left(\mathbb{R}^{3}, \mathbb{R}^{s}\right)$ such that the constant system is, as usual,

$$
\theta=d u-p d x-q d y-r d z=0
$$

where $u, p, q, r$ are $\mathbb{R}^{s}$-valued, and such that $X_{1}$ is locally given by an equation

$$
r=F(x, y, z, u, p, q)
$$

where $F$ is defined in convex open set in $\mathbb{R}^{3+3 s}$. The integers $n$ and $\ell$ are 3 and 1 , respectively, and thus the relevant dimension range is $0 \leqq q<2$.

Suppose first that $q=0$ and $\partial I^{1}$ is comprised of the two points $\Upsilon_{i}=$ $\left(x_{i}, y_{i}, z_{i}, u_{i}, p_{i}, q_{i}, r_{i}\right), i=0,1$, and where $r_{i}=F\left(x_{i}, y_{i}, z_{i}, u_{i}, p_{i}, q_{i}\right)$. We want to find a piecewise- $C^{1}$ arc $\gamma(t)$ with $\gamma(0)=\gamma_{0}, \gamma(1)=\gamma_{1}$ and satisfying

$$
\left\{\begin{aligned}
r(t) & =F(x(t), y(t), z(t), u(t), p(t), q(t)), \\
u^{\prime}(t) & =p(t) x^{\prime}(t)+q(t) y^{\prime}(t)+r(t) z^{\prime}(t) .
\end{aligned}\right.
$$

Given $x(t), y(t), z(t), u(t)$ these are a set of finite (i.e., not differential) equations for $p(t), q(t), r(t)$. Moreover, these finite equations are underdetermined. Thus we may expect that in general-i.e., when suitable open general position conditions are satisfied-we may solve these equations.

In fact, in the case $q=0$ we may always solve the equations (8). If $\left(x_{0}, y_{0}, z_{0}\right) \neq\left(x_{1}, y_{1}, z_{1}\right)$, then we may assume that $x_{0} \neq x_{1}$ and $y_{0}=$ $y_{1}, z_{0}=z_{1}$. We may then take an arbitrary arc $x(t)$ with $x(0)=x_{0}$, $x(1)=x_{1}, x^{\prime}(t) \neq 0$, and set $y(t)=z(t)=0$. We then choose a function $u(t)$ with $u(0)=u_{0}, u(1)=u_{1}, u^{\prime}(0)=p_{0} x_{0}^{\prime}, u^{\prime}(1)=p_{1} x_{1}^{\prime}$ and then set $p(t)=u^{\prime}(t) / x^{\prime}(t)$. Finally we may choose $q(t)$ to be any arc with $q(0)=q_{0}$, $q(1)=q_{1}$ to have a solution to (8). Of course, we must take care to insure that $(x(t), y(t), z(t), u(t), p(t), q(t))$ remains in the domain of definition of $F$, and this may be done by subdividing the interval and using a piecewise- $C^{1}$ arc.

If, on the other hand, $\left(x_{0}, y_{0}, z_{0}\right)=\left(x_{1}, y_{1}, z_{1}\right)$, then a similar argumentin fact with the same notation-applies.

Remark that in general the case $q=0$ of (4) follows from Chow's theorem (see Chapter 1 of [2]), and indeed one may view (4) as an extension of that result.

More interesting is the case $q=1$. If $\square$ is the unit square $0 \leqq s \leqq 1$, $0 \leqq t \leqq 1$ in the $(s, t)$ plane, then we must find a mapping

$$
(s, t) \rightarrow(x(s, t), y(s, t), z(s, t), u(s, t), p(s, t), q(s, t), r(s, t))
$$


satisfying

$$
\left\{\begin{array}{l}
\text { (i) } \quad r=F(x, y, z, u, p, q), \\
\text { (ii) } \quad u_{s}=p x_{s}+q y_{s}+r z_{s}, \\
\text { (iii) } \quad u_{t}=p x_{t}+q y_{t}+r z_{t},
\end{array}\right.
$$

and where (9) is given on the boundary of the square such that $(10)$ is satisfied there. If $x(s, t), y(s, t), z(s, t), u(s, t)$ are given arbitrarily, then (10) is a determined set of finite equations. Equations (10)-(ii) and (10)-(iii) are analogous to the equations encountered in Thom's result, and we may seek to adapt his arguments to our situation. In fact, this may be done provided that the boundary conditions are in general position. Thus, assume that the image of $\partial \square$ is in general position in the sense that we may choose coordinates so that the projection of $\partial \square$ to the $(x, y, z)$ space is the unit square in the $x$, $y$ plane. Then we may replace $s, t$ by $x, y$ and seek to determine a mapping of the special form

$$
(x, y) \rightarrow(x, y, 0, u(x, y), p(x, y), q(x, y), r(x, y))
$$

such that (10) is satisfied. Equations (10)-(ii) and (10)-(iii) reduce to those in Thom's theorem, with no equations involving $r$ since $z_{x}=z_{y}=0$. Having solved for $p$ and $q$, equation (10)-(i) then gives us $r$. Moreover, if $u, p, q$ satisfy the boundary conditions, then so does $r$. Summarizing, if the boundary conditions are in general position, then Thom's arguments may be used to establish (5) in the case $s_{0}=s_{1}=s_{2}, s_{3}=0$.

We now point out that the arguments continue to apply-and indeed are "easier"-if $s_{3} \neq 0$. In this case we may decompose $\mathbb{R}^{s}=\mathbb{R}^{s_{3}} \oplus \mathbb{R}^{s-s_{3}} \quad(s=$ $\left.s_{0}=s_{1}=s_{2}\right)$ and correspondingly write $v \in \mathbb{R}^{s}$ as $v=\left(v^{\prime}, v^{\prime \prime}\right)$. The equation (7) is then replaced by

$$
r^{\prime \prime}=F\left(x, y, z, u, p, q, r^{\prime}\right),
$$

and the argument proceeds as before. It is "easier" in the sense that not only $u$ but also $r^{\prime}$ may be prescribed arbitrarily, subject to open conditions in the $C^{1}$-topology plus the boundary conditions.

In summary, then, (i) above might be provable by arguments of the type used in [10]. It remains to explain how the absence of real Cartan characteristics allows us to achieve general position for the boundary conditions.

We recall from Chapter $\mathrm{V}$ in [2] that characteristics arise when the rank of polar equations of integral elements falls below the maximum. To say that $\mathscr{J}_{0}$ has no real Cartan characteristics in the range $0 \leqq q<n-\ell$ therefore means that in this range the polar equations have constant rank. If $E^{q}$ is an integral element of dimension $q \geqq 1$, then this rank is $s_{1}+\cdots+s_{q}$ (the case $q=0$ is trivial, and all $E^{0}$ are non-characteristic).

As examples, we note that this condition is satisfied for the contact system on $J^{k}\left(\mathbb{R}^{n}, \mathbb{R}^{s}\right)$, and indeed for any system whose symmetry group acts transitively on the $q$-dimensional integral elements for $0 \leqq q<n-\ell$. The condition is clearly generic and open. On the other hand, for the exterior differential system arising from the $\bar{\partial}$-operator in $m$ complex variables, we have $n=2 m$ and 
$\ell=m$; moreover, if $m \geqq 3$ there are real Cartan characteristics in dimensions $2 \leqq q<m$ even though the system itself is elliptic.

The way in which the non-characteristic condition arises is this: Referring to the special case $s=s_{0}=s_{1}=s_{2}, s_{3}=0$ discussed above, suppose we have $f: \partial \square^{2} \rightarrow U \subset X_{1}$. Then we wish to deform $f$ in a neighborhood of a point $p \in \partial \square$ in order to get it in general position at $p$. The way to do this is to construct a little piece of integral surface near $p$

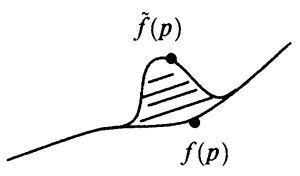

and replace $f$ by $\tilde{f}$ given on the other side of the piece of integral surface. Now since we are in the range $0 \leqq q<n-\ell$, constructing the piece of integral surface involves solving finite, not differential equations, ${ }^{4}$ as we saw above. Infinitesimally, constructing the piece of integral surface amounts to solving the polar equations

$$
\Theta(\xi, v)=0, \quad \Theta \in \mathscr{F}_{1}^{2},
$$

where $\xi$ is the tangent to $f(\partial \square)$ at $f(p)$ and $v \in T_{f(p)}\left(X_{1}\right)$. So long as these equations do not have jumping rank, we may smoothly do this. In terms of the coordinates used above, the absence of real characteristics means that for any (not just a generic) choice of coordinates $(x, y, z)$ in $\mathbb{R}^{3}$ we may solve for $r$ as in (7).

In concluding this discussion, we mention that we are not saying the presence of real Cartan characteristics necessarily provides an obstruction to the validity of (4). What we are saying is that such characteristics provide singularity issues that go beyond those that arose in Thom's paper, and which clearly merit further investigation.

6.3. Moment conditions. We retain the notation of the preceding section, and shall work locally. In building up integral manifolds the critical dimension is $n-\ell$, for it is here that the transition is made from "strip conditions" to what might be called "honest differential equations". ${ }^{5}$ A very natural question to consider is the Dirichlet problem in the critical dimension:

Given an integral manifold

$$
f: S^{n-\ell} \rightarrow X,
$$

\footnotetext{
${ }^{4}$ What is going on here is that we are discussing the classical strip conditions used to prescribe the boundary values of a PDE in terms of a certain number of arbitrary functions and their derivatives. Since we are in the range $0 \leqq q<n-\ell$ there are no conditions imposed on the derivatives of these functions, in the sense that the number of equations is insufficient to impose such conditions provided that we are in general position.

${ }^{5}$ Strip conditions for $q$-dimensional integral manifolds in the range $0 \leqq q \leqq n-\ell$ are parametrized by $s=s_{q}$ arbitrary functions of $q$-variables (arbitrary means subject to open conditions in the $C^{1}$-topology). In contrast, the integral manifolds of dimension $n-\ell+1$ are given by $s_{n-\ell+1}<s$ arbitrary functions of $n-\ell+1$ variables; the remaining $s-s_{n-\ell+1}$ functions will be subject to non-trivial closed conditions given by the imposition of a PDE system.
} 
what are the conditions that it extend to an integral manifold

$$
F: B^{n-\ell+1} \rightarrow X .
$$

Here, $S^{n-\ell}$ and $B^{n-\ell+1}$ are the standard sphere and ball with $\partial B^{n-\ell+1}=$ $S^{n-\ell}$; since we are working locally we may assume that a topological extension $F$ exists. The "moment conditions" are the homological obstruction to the Dirichlet problem. More formally,

Definition. Let $K$ be the kernel of the natural mapping

$$
H_{n-\ell, \mathscr{I}}(X) \rightarrow H_{n-\ell}(X) .
$$

Then the moment conditions are

$$
K^{*}=\operatorname{Hom}(K, \mathbb{R}) .
$$

There is an obvious interesting mapping

$$
\mu: \mathscr{C} \rightarrow K^{*}
$$

from conservation laws to moment conditions, and in this section we will discuss a number of examples of $\mu$. Roughly speaking, it seems that $\mu$ should be injective and will sometimes, but not always, be an isomorphism. ${ }^{6}$ In these examples, which are drawn from those in Section 1, we will work locally so that $H_{n-\ell}(X)=0$ and (1) reduces to

$$
\mu: \mathscr{C} \rightarrow \operatorname{Hom}\left(H_{n-\ell, \mathcal{F}}(X), \mathbb{R}\right) .
$$

We will also follow the notation of that section, and will therefore replace $X$, $\mathscr{I}$ and $\mathscr{C}$ by $X_{0}, \mathscr{I}_{0}$ and $\mathscr{C}_{0}$ as we shall be working with the non-prolonged systems.

Example 1 (the contact system). In this case $\operatorname{dim} X_{0}=2 n+1$ and $\mathscr{I}_{0}$ is generated by

$$
\theta=d u-\sum_{i=1}^{n} p_{i} d x^{i}
$$

Also, since $\ell=0$ we should think of $H_{n, \mathcal{F}_{0}}\left(X_{0}\right)$ as "compactly supported homology". Thus, "cycles" are given by integral manifolds $x \rightarrow(x, u(x), D u(x))$ where $u$ is compactly supported, and no two cycles are homologous since there are no $(n+1)$-dimensional integral manifolds. The space of conservation laws is given by functionals on integral manifolds, and (2) amounts to the mapping

$$
\lambda \rightarrow \int \lambda(x, u(x), D u(x)) d x
$$

where $u(x)$ is compactly supported. To prove that $\mu$ is an isomorphism means to show that:

$$
\int \lambda(x, u(x), D u(x)) d x=0
$$

\footnotetext{
${ }^{6}$ We have not proved that it ever fails to be an isomorphism; this seems to require additional foundational work.
} 
for all compactly supported $u \Rightarrow$ the class of $\lambda(x, u, p) d x$ is zero in $\bar{H}_{0}^{n}$-i.e., $\lambda d x$ is a divergence, and

$$
\int \lambda(x, u(x), D u(x)) d x=\int \lambda(x, \tilde{u}(x), D \tilde{u}(x)) d x
$$

for all $\lambda \Rightarrow u=\tilde{u}$.

Both of these are elementary to prove.

Example 2 (the symplectic system). In this case $\operatorname{dim} X_{0}=2 n, n \geqq 2$ and $\mathscr{J}_{0}$ is generated by

$$
\Theta=\sum d p_{i} \wedge d x^{i}
$$

Also, from Section 1 we recall that $n-\ell=1$ and that $\mathscr{C}_{0}$ is one dimensional and generated by any 1 -form $\theta$ satisfying

$$
d \theta=\Theta \text {. }
$$

The space $H_{1, \mathcal{F}_{0}}\left(X_{0}\right)$ is given by closed curves modulo the equivalence relation that $\gamma \sim 0$ in case $\gamma=\partial \Gamma$ where $\Gamma$ is a piecewise- $C^{1}$ 2-chain whose simplices are Lagrangian surfaces. We will prove two special cases towards showing that (2) is an isomorphism.

To do this we first consider the case where $\gamma$ is the image of a mapping

$$
f: S^{1} \rightarrow X_{0}
$$

where $S^{1}$ is the unit circle in $\mathbb{R}^{2}$. We will show that $f$ extends to a mapping

$$
F: B^{2} \rightarrow X_{0}
$$

satisfying

$$
F^{*} \Theta=0
$$

if and only if the moment condition

$$
\int_{S^{1}} f^{*} \theta=0
$$

is satisfied.

Choose any base point $P_{0} \in S^{1}$ and set

$$
u(P)=\int_{P_{0}}^{P} f^{*} \theta .
$$

This defines a function $u$ on $S^{1}$ and we have a diagram

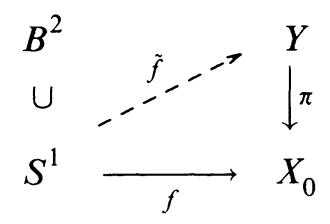


where $Y=X_{0} \times \mathbb{R}$ and $\tilde{f}=(f, u)$ (cf. Section 1). By construction,

$$
\tilde{f}^{*}(d u-\theta)=0
$$

and we may then extend $\tilde{f}$ to a mapping

$$
\tilde{F}: B^{2} \rightarrow Y
$$

satisfying

$$
\tilde{F}^{*}(d u-\theta)=0
$$

by the filling in problem for contact systems (cf. Thom [10]). For $F=\pi \circ \tilde{F}$ we have

$$
F^{*} \Theta=-\tilde{F}^{*}(d(d u-\theta))=0
$$

as required.

A slight extension of this argument deals with two mappings

$$
f_{i}: S^{1} \rightarrow X_{0}, \quad i=0,1,
$$

which satisfy

$$
\int_{S^{1}} f_{0}^{*} \theta=\int_{S^{1}} f_{1}^{*} \theta .
$$

We then want to fill in the cylinder; i.e., to find a mapping

$$
F: S^{1} \times I \rightarrow X_{0}
$$

such that $F\left|S^{1} \times\{0\}=f_{0}, F\right| S^{1} \times\{1\}=f_{1}$ and

$$
F^{*} \Theta=0 \text {. }
$$

As above, we consider $\tilde{Y}=S^{1} \times X_{0}$ and the diagram

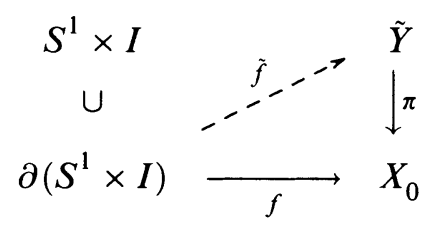

where $f$ is given by $f_{0}, f_{1}$ and the lifting $\tilde{f}$ exists by virtue of (3) (the $u$ coordinate is now periodic with the same period $\left.\int_{S^{1}} f_{i}^{*} \theta\right)$. By construction, $\tilde{f}^{*}(d u-\theta)=0$ and so again by Thom's theorem we may extend $\tilde{F}$ to

$$
\tilde{F}: S^{1} \times I \rightarrow \tilde{Y}
$$

satisfying $\tilde{F}^{*}(d u-\theta)=0$.

The argument when $\gamma$ is an arbitrary 1-cycle is more complicated and will be omitted. 
Example 3 (CR system). This is the example from Section 1 where $X_{0}$ is a complex manifold and $\mathscr{F}_{0}$ is generated by the 2-forms $\alpha+\bar{\alpha}, \alpha \in \Omega^{2,0}\left(X_{0}\right)$. Since we are working locally we may assume that $X_{0}$ is a contractible, Stein open set in $\mathbb{C}^{m}, m \geqq 2$. Then $n=2, \ell=1$ and the moment conditions are the linear functionals on $H_{1, \mathcal{F}_{0}}\left(X_{0}\right)$. On the other hand, as we saw in Section 1 the conservation laws are given by

$$
\mathscr{C}_{0} \cong\{\text { holomorphic } 1 \text {-forms } \psi / d \text { (holomorphic functions) }\}
$$

and we are interested in the question of whether the mapping

$$
\mu_{0}: \mathscr{C}_{0} \rightarrow \operatorname{Hom}\left(H_{1, \mathscr{J}_{0}}\left(X_{0}\right), \mathbb{R}\right)
$$

is an isomorphism. As in the symplectic example just considered, the crucial result is the following theorem of Harvey-Lawson [6]:

Given a mapping

$$
f: S^{1} \rightarrow X_{0}
$$

satisfying

$$
\int_{S^{1}} f^{*} \psi=0
$$

for all $\psi \in \mathscr{C}_{0}$, then $f$ extends to a holomorhic mapping of the disc.

As discussed in [6], this result may be extended to show that $\mu_{0}$ in (2) is an isomorphism.

Example 4 (associative geometry). This is the exterior differential system given by the final example in Section 1. In this case $n=3, \ell=1$ and so the crucial homology group is $H_{2, \mathscr{F}_{0}}\left(X_{0}\right)$. As we saw in the earlier discussion of this example,

$$
\operatorname{dim} \mathscr{C}_{0}=21
$$

and we shall see that this strongly suggests (but does not quite prove) that

$$
\mu_{0}: \mathscr{C}_{0} \rightarrow \operatorname{Hom}\left(H_{2, \mathscr{F}_{0}}\left(X_{0}\right), \mathbb{R}\right)
$$

fails to be surjective. ${ }^{7}$

Let $E^{3} \subset \mathbb{R}^{1}$ be an associated 3-plane with $B^{3} \subset E^{3}$ the unit ball having boundary $S^{2}=\partial B^{3}$. We seek the equations on sections of the normal bundle to $B^{3}$ in $\mathbb{R}^{7}, T\left(\mathbb{R}^{7}\right) / T\left(B^{3}\right) \cong\left(\mathbb{R}^{7} / E^{3}\right) \times B^{3}$, that express the conditions that the corresponding infinitesimal variation of $B^{3}$ remain an integral manifold of $\mathscr{I}_{0}$. In fact, letting $F$ denote the trivial bundle with fiber $\mathbb{R}^{7} / E^{3} \cong \mathbb{R}^{4}$, the linear equations for variation of $B^{3}$ as an integral manifold of $\mathscr{J}_{0}$ are just the kernel of the Dirac operator

$$
D: F \rightarrow F
$$

(cf. Harvey-Lawson [7]).

\footnotetext{
${ }^{7}$ This argument only depends on the fact that $\operatorname{dim} \mathscr{C}_{0}<\infty$.
} 
Now in general if we have a linear elliptic operator, for convenience still denoted by (4), over the $n$-ball $B \subset \mathbb{R}^{n}$, then for solutions $u$ of $D$ and $v$ of the adjoint operator $D^{*}$

$$
\int_{\partial B}(u, v)=\int_{B}(D u, v)+\int_{B}\left(u, D^{*} v\right)=0 .
$$

In other words, the boundary values of solutions to the adjoint equation $D^{*} v=$ 0 give an infinite number of independent moment conditions on boundary values of solutions to the equation $D u=0 .^{8}$

Applying this to variations of $B^{3}$ as an integral manifold of $\mathscr{I}_{0}$, it follows that there are an infinite number of independent conditions imposed on a small perturbation $S^{\prime}$ of $S^{2}$ that $S^{\prime}$ fill in to an integral manifold. On the other hand, the conservation laws of the system $\mathscr{I}_{0}$ provide only a finite number of moment conditions.

There are two ways in which this discussion is incomplete and therefore fails to provide an example where (2) is not an isomorphism. The first is that we have only discussed the moment conditions to fill in $S^{\prime}$ with a $C^{1}$ integral manifold. Conceivably, to realize $S^{\prime}$ as the boundary of a piecewise- $C^{1}$ chain whose individual simplices are integral manifolds of $\mathscr{J}_{0}$ might provide enough additional flexibility to enable (2) to be an isomorphism. Because of regularity for elliptic equations this seems to us unlikely.

The other possibility is that when we pass to the infinite prolongation of $\mathscr{J}_{0}$, then $\bar{H}_{0}^{2}$ increases to give an infinite-dimensional $\bar{H}^{2}$ which accounts for all the moment conditions. What would probably have to happen is that the infinite number of conservation laws for the linearized exterior differential system (4) somehow survive to give conservation laws for $\mathscr{I}$ provided that we allow higher order jet dependence. This also seems to us unlikely.

\section{REFERENCES}

1. I. Anderson, The variational bicomplex, Notes from Dept. of Mathematics at Utah State University, 1990.

2. R. Bryant, S. S. Chern, R. Gardner, H. Goldschmidt, and P. Griffiths, Exterior differential systems, Math. Sci. Res. Inst. Publ., vol. 18, Springer-Verlag, Berlin and New York, 1991.

3. R. Bryant, Metrics with exceptional holonomy, Ann. of Math. (2) 126 (1987), 525-576.

4. L. Dickey, On exactness of variational bicomplex, preprint from the Univ. of Oklahoma, 1991.

5. I. M. Gelfand and L. Dikii, Asymptotic behavior of the resolvent of Sturm-Liouville equations and the algebra of Korteweg-de Vries equations, Uspekhi Mat. Nauk 30 (1975), no. 5, 67100.

6. R. Harvey and H. B. Lawson, On boundaries of complex analytic varieties, I, Ann. of Math. (2) 102 (1975), 223-290.

7. __ Calibrated geometries, Acta Math. 148 (1982), 47-157.

8. Yu. I. Manin, Algebraic aspects of nonlinear differential equations, J. Soviet Math. 11 (1979), $1-122$.

${ }^{8}$ It is a general fact that for a linear PDE, solutions to the adjoint equation give conservation laws for the original equation. This is the basis for the observation above. 
9. P. Olver, Applications of Lie groups to differential equations, Springer-Verlag, Berlin and New York, 1986.

10. R. Thom, Remarques sur les problèmes comportant des inéqualities différentielles globales, Bull. Soc. Math. France 87 (1959), 455-461.

11. T. Tsujishita, On variational bicomplexes associated to differential equations, Osaka J. Math. 19 (1982), 311-363.

12. _ _ Homological method of computing invariants of systems of differential equations, Preprint available from Dept. of Mathematics, Osaka Univ., 1991.

13 A. M. Vinogradov, The $\mathscr{C}$-spectral sequence, Lagrangian formalism and conservation laws I, II, J. Math. Anal. Appl. 100 (1984), 1-129.

14. __ Local symmetries and conservation laws, Acta Appl. Math. 2 (1984), 21-78.

15. __ Geometry of nonlinear differential equations, J. Soviet Math. 17 (1981), 1624-1649.

16. R. Bryant and P. Griffiths, Characteristic cohomology of differential systems (II): Conservation laws for a class of parabolic systems, Duke Math. J. (to appear).

Department of Mathematics, Duke University, Durham, North Carolina 27708-0320

E-mail address: bryant@math. duke.edu

Institute for Advanced Study, Princeton, New Jersey 08540-0631

E-mail address: pg@math.ias.edu 